\title{
Perspektivenübernahme als eine Kompetenz historischen Verstehens
}

\author{
Dissertation \\ zur Erlangung des mathematisch-naturwissenschaftlichen Doktorgrades \\ „Doctor rerum naturalium“ \\ der Georg-August-Universität zu Göttingen
}

vorgelegt von

Ulrike Hartmann (geb. Kuhlmann)

aus Herdecke

Göttingen 2008 
Referent: Prof. Dr. Marcus Hasselhorn

Korreferent: Prof. Dr. Michael Sauer

Tag der mündlichen Prüfung: 24.10.2008 
Wäre der Wille eines jeden Menschen frei, das heißt, könnte jeder Mensch so handeln, wie er gerade will, dann würde die Geschichte aus einer Reihe von zusammenhanglosen Zufälligkeiten bestehen.

L. N. Tolstoi, Krieg und Frieden 


\section{DANKE...}

Bei folgenden Menschen möchte ich mich für die Unterstützung während der letzten Jahre ganz herzlich bedanken:

Die Betreuer meiner Arbeit, Marcus Hasselhorn und Michael Sauer, haben mich mit vielen hilfreichen Ratschlägen und Ideen durch das Experiment begleitet, Kompetenzen historischen Verstehens psychologisch zu untersuchen. Sie haben mir viel Vertrauen entgegengebracht und ich habe mich kontinuierlich dazu ermuntert gefühlt, mit dieser Arbeit meinen eigenen Interessen nachzugehen. Besonders danke ich ihnen für die Geduld, mit der sie die verschiedenen Versionen der Zeitschriftenbeiträge ein ums andere Mal sowohl gründlich als auch zügig gelesen haben, und mir dadurch eine publikationsbasierte Promotion ermöglichten.

Meine Kolleginnen und Kollegen des GRK 1195 haben die letzten drei Jahre für mich zu einer unvergesslichen Zeit werden lassen. Besonders Andju Sara Labuhn hat durch ihre wertvollen Hinweise und ihren kritischen Blick einen großen Teil zum Gelingen dieser Arbeit beigetragen und mich als Freundin durch die Höhen und Tiefen der Promotionszeit begleitet. Die Zusammenarbeit mit Matthias Martens im Geschichtsprojekt hat meine Sicht auf interdisziplinäres Arbeiten maßgeblich beeinflusst und mir gezeigt, wie viel Spaß Diskussionen über unterschiedliche Forschungszugänge und die Herausforderungen und Potentiale interdisziplinärer Wissenschaft machen. Bei ihm möchte ich mich auch für seine nützlichen Anmerkungen zum Theorieteil der Arbeit bedanken. Darüber hinaus ist es unmöglich, alle Begebenheiten im GRK aufzuzählen, durch die ich mich ermutigt, verstanden und unterstützt gefühlt habe. Stellvertretend möchte ich mich bei Klaudia Schulte, Jasmin Warwas und Bastian Funken bedanken, vor allem für die Aufmunterung und Ablenkung während der zeitweise frustrierenden Review-Prozesse.

Für die Unterstützung bei der Durchführung und Interpretation der Latenten KlassenAnalysen danke ich herzlich Jürgen Rost und Johannes Hartig.

Sarah Nieroba war mir vor allem während der Endphase dieser Arbeit eine wertvolle Gesprächs- und Laufpartnerin und hat durch ihr genaues Lesen noch so manchen Flüchtigkeitsfehler entdeckt.

Meine Eltern Marlene und Heinz-Peter Kuhlmann sowie meine Brüder Julian und Paul Kuhlmann haben mich - wie auf meinem gesamten Weg - auch durch die Zeit der Promotion verständnisvoll begleitet und sind mir in allen Lebenslagen eine große Unterstützung. Bei meinem Mann Ullrich möchte ich mich für so vieles bedanken, doch das lässt sich nur schwer - wenn überhaupt - in Worte fassen.

\section{THANK YOU...}

My ideas that are expressed in this dissertation were influenced a lot by discussions with Robert L. Selman, Angela Bermudez and Alan Stoskopf. I am very grateful to you, Bob, for giving me the opportunity to spend three months with your team and to learn more about the fascinating work on social awareness and the Facing History program. Angela and Alan, your thoughtful work on the Historical Understanding Measure has affected my thoughts on what is history and how to measure it in innumerable ways. Thank you all for making me feel so welcome! 


\section{Inhalt}

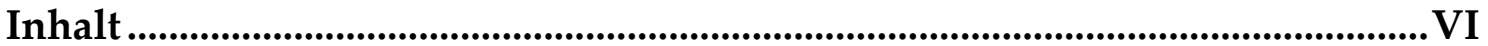

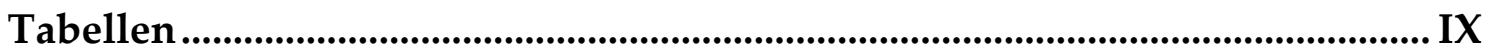

Abbildungen ……............................................................................................ IX

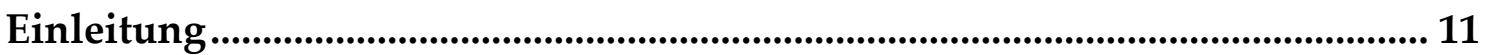

1. Perspektivenübernahme als Bestandteil von Kompetenzmodellen historischen Verstehens .................................................................................. 15

1.1 Vorbemerkungen .........................................................................................................15

1.2 Mit Perspektivität in der Geschichte umgehen und Fremdverstehen leisten im Kompetenzmodell von Michael Sauer..................................................................16

1.3 Perspektivenübernahme als Bestandteil von Interpretationskompetenz, narrativer Kompetenz und Urteilskompetenz im Modell von Peter Gautschi.18

1.4 Kompetenz zur Reflexion und Erweiterung des Welt- und Fremdverstehens im Kompetenz-Strukturmodell historischen Denkens der Gruppe FUER Geschichtsbewusstsein

1.5 Perspektivenübernahme als Bestandteil von Bildungsstandards für das Fach Geschichte

1.5.1 Perspektivenübernahme als Teil des National Curriculum for History in England ...22

1.5.2 Perspektivenübernahme als Teil der National Standards for United States History.. 24

1.5.3 Perspektivenübernahme als Teil von Bildungsstandards für das Fach Geschichte

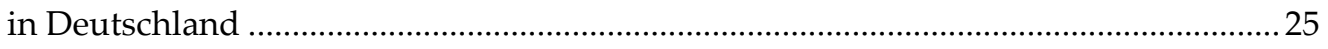

1.6 Historical Empathy im Modell von Rosalyn Ashby und Peter Lee. 28

1.7 Bewertung der Kompetenzmodelle im Hinblick auf ihre Anschlussfähigkeit für die empirische Erforschung historischer Perspektivenübernahme 31

2. Perspektivenübernahme als psychologisches Konstrukt mit Anschlussmöglichkeiten für die Erforschung historischen Verstehens ..... 33

2.1 Vorbemerkungen .............................................................................................................33

2.2 Das Modell der sozialen Perspektivenkoordination nach Robert L. Selman ...33

2.3 Perspektivenübernahme als kontextabhängige Kompetenz .................................36

2.3.1 Kontextabhängigkeit von Perspektivenübernahme und sozio-moralischem Urteil

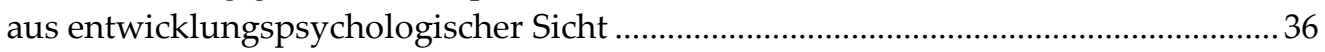

2.3.2 Perspektivenübernahme als multidimensionale soziale Kompetenz ..............................38

2.4 Soziale Perspektivenübernahme im historischen Kontext - Versuch einer

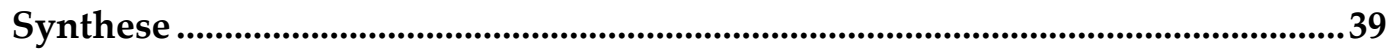

2.4.1 Fachdidaktische Kompetenzannahmen und das Modell zur sozialen

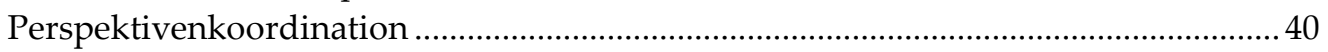

2.4.2 Historische Perspektivenübernahme als multidimensionales Konstrukt? ..................... 42

2.4.3 Ziele historischer und sozialer Perspektivenübernahme ................................................. 42

3. Forschungsziele.............................................................................................................. 44 
4. Analysen zur Qualität eines neuen Messinstrumentes zur Erfassung historischer Perspektivenübernahme.

4.1 Historical Perspective Taking: A Standardized Measure for an Aspect of

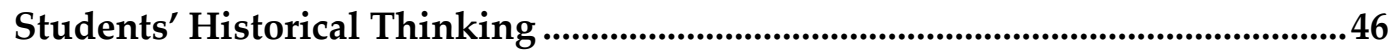

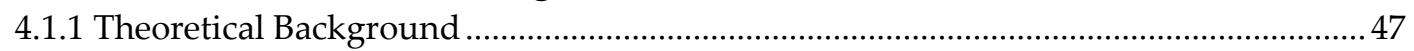

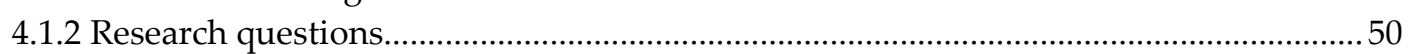

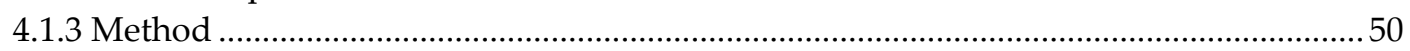

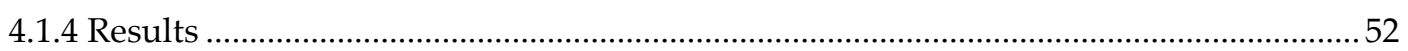

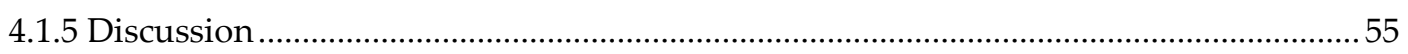

4.2 Kompetenzprofile historischer Perspektivenübernahme in Klasse 7 .................58

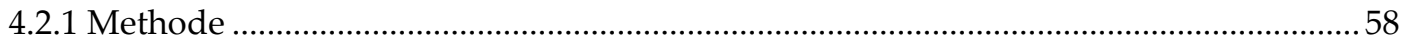

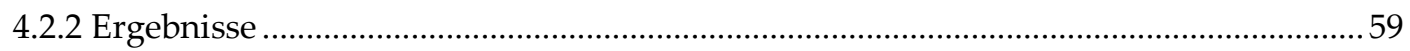

4.3 Vergleichende Zusammenfassung der Kompetenzprofile

in Klasse 7 und 10

5. Perspektivenübernahme als Kompetenz für den Geschichtsunterricht: theoretische und empirische Zusammenhänge zwischen fachspezifischen und sozial-kognitiven Schülermerkmalen............................................ 65

5.1 Einleitung .65

5.1.1 Perspektivenübernahme als Bindeglied zwischen sozialer Kompetenz und historischem Verstehen.....

5.1.2 Zusammenhänge zwischen historischer Perspektivenübernahme, Motivation und Leistung im Geschichtsunterricht.

5.2 Ziel der Studie ....................................................................................................................70

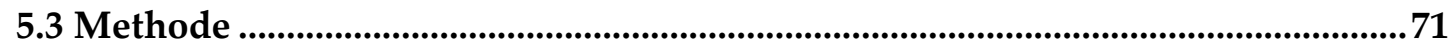

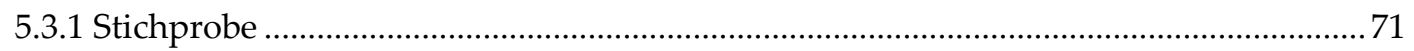

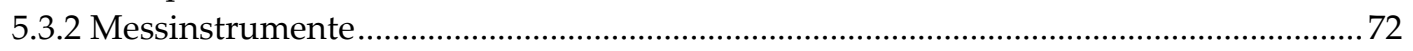

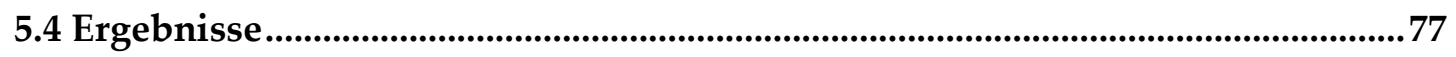

5.5 Zusammenfassung der Ergebnisse ................................................................................. 81

5.6 Mögliche Wirkzusammenhänge sozial-kognitiver und geschichtsspezi-

fischer Schülermerkmale ...................................................................................................83

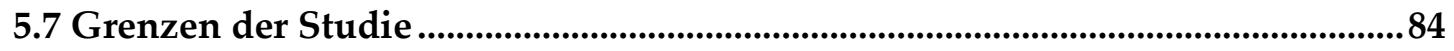

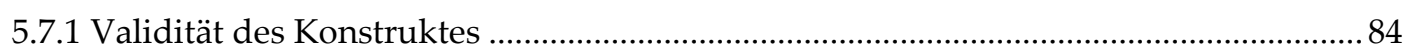

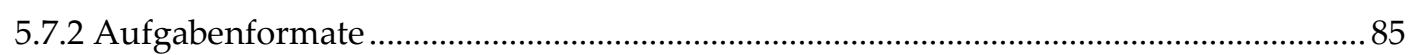

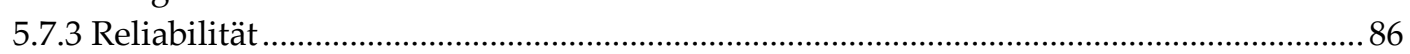

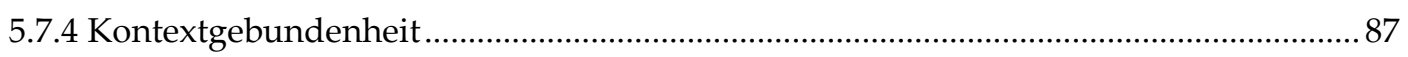

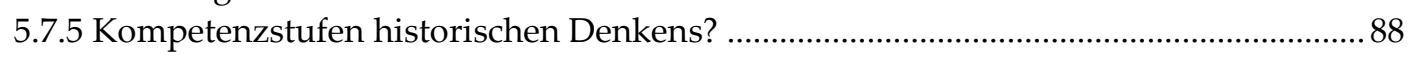

6. Perspektivenübernahme als sozial-kognitive Kompetenz historischen

Verstehens - Diskussion und Ausblick .................................................................. 90

6.1 Theoretische und empirische Erträge.......................................................................90

6.2 Implikationen für die Messung historischer Kompetenzen...................................92

6.2.1 Interdisziplinarität als Herausforderung und Chance ………….................................93

6.2.2 Von Kompetenzausprägungen zu Kompetenzstufen historischen Verstehens............ 96

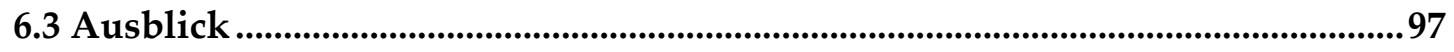




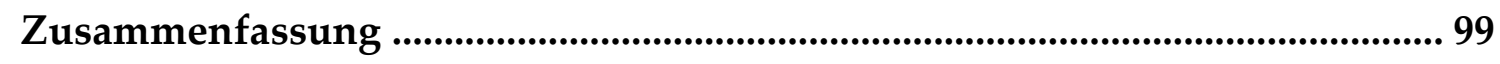

Abstract (english) ....................................................................................................... 100

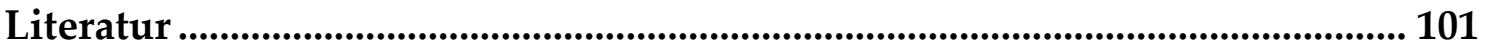

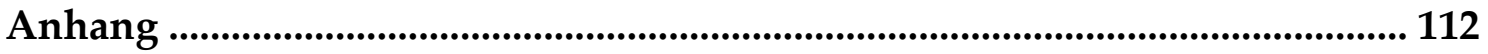

A.1 112

A.2

A.3

A 4 -

A 5 . 


\section{Tabellen}

Tabelle 1: Zusammenfassung des Modells für Historical Empathy ............................ 30

Tabelle 2: Results of the principal component analysis............................................. 52

Tabelle 3: Results of the latent class analysis ......................................................... 53

Tabelle 4: Expected class membership probabilities .................................................. 55

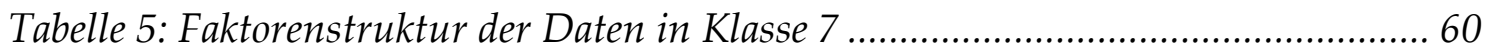

Tabelle 6: Vergleich verschiedener Latent Class Models (LCM)................................. 61

Tabelle 7: Interne Konsistenzen der eingesetzten Messinstrumente ............................ 72

Tabelle 8: Items zur Erfassung historischer Perspektivenübernahme.......................... 74

Tabelle 9: Deskriptive Statistiken zu den eingesetzten Skalen................................... 77

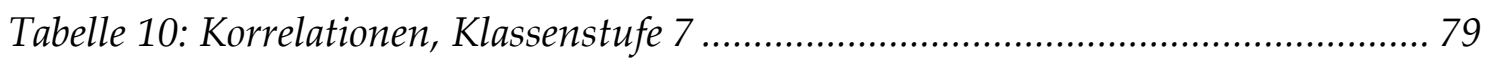

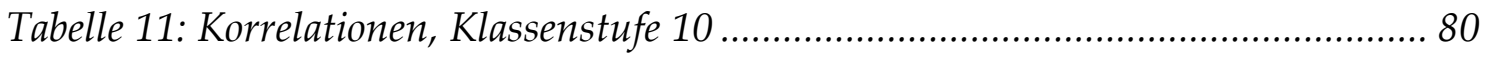

\section{Abbildungen}

Abbildung 1: Item score profiles of the HPT measure............................................... 54

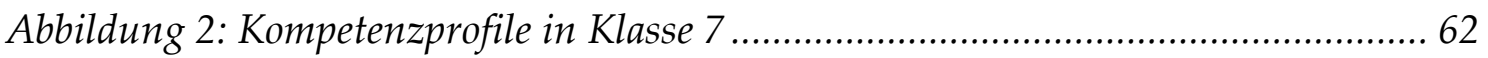




\section{Einleitung}

Die Debatte über Bildungsstandards, angestoßen durch die Expertise, die im Auftrag des Bundesministeriums für Bildung und Forschung erstellt wurde (Klieme et al., 2003), betrifft die gesamte schulische Bildungslandschaft in Deutschland. Zentrale Forderung ist die Abwendung von der Input-Orientierung (üblicherweise in Form von Lehrplänen, die Unterrichtsinhalte vorschreiben) und die gleichzeitige Hinwendung zu einer Output-Orientierung (in Form von anvisierten Schülerkompetenzen). Je nachdem, welches Schulfach man betrachtet, hat die Debatte mehr oder weniger starke Auswirkungen. Für die Standardentwicklung insbesondere in den Hauptfächern - Mathematik, Deutsch und die erste Fremdsprache - wurde mit dem Institut für Qualitätsentwicklung im Bildungswesen (IQB) eine Einrichtung geschaffen, die als Dienstleistungseinrichtung aller Bundesländer sowohl die Formulierung von Bildungsstandards als auch die daran angelehnte Messung von Schülerkompetenzen vorantreibt. Eine zweite Gruppe von Fächern bilden die Naturwissenschaften. Biologie, Physik und Chemie schließen bezüglich der theoretischen und empirischen Kompetenzforschung nach und nach zu den Hauptfächern auf. Dazu trägt auf nationaler Ebene unter anderem das Institut für die Pädagogik der Naturwissenschaften (IPN) bei, welches die notwendige methodische Expertise bündelt und Forschungsvorhaben an verschiedenen Universitäten koordiniert um Schülerkompetenzen in diesen Fächern zu erfassen (z.B. Biologie im Kontext: Bayrhuber et al., 2007). Darüber hinaus ist ein internationaler Trend festzustellen, den Naturwissenschaften in ihrer Bedeutung für schulisches Lernen einen besonderen Stellenwert einzuräumen. Diese Fächer stellten den Schwerpunkt der PISA-Studie 2006 dar (Prenzel et al., 2007). Auch vom IQB werden mittlerweile Tests zur Überprüfung der Bildungsstandards in den Naturwissenschaften entwickelt.

Eine dritte Gruppe von Fächern, die sich für andere Zwecke als die der Argumentation dieser Arbeit selbstverständlich weiter ausdifferenzieren ließe, ist bisher nicht unmittelbar von der Umsetzung der Bildungsstandards betroffen. $\mathrm{Zu}$ dieser Gruppe zählt das Fach Geschichte. In Deutschland existieren bisher allenfalls erste Vorschläge für Kompetenzmodelle, die als verbindlicher Ausgangspunkt für die Messung von Schülerkompetenzen und die Überprüfung von Bildungsstandards auf nationaler Ebene dienen könnten (z.B. Körber, Schreiber \& Schöner, 2007; Sauer, 2006). Trotzdem lässt sich dieses Fach durchaus als kompetenzorientiert bezeichnen. Schulisches Lernen im Geschichtsunterricht dient mitnichten nur der Einprägung von Jahreszahlen, histori- 
schen Ereignissen und berühmten Persönlichkeiten. Vielmehr sind die dominierenden Begriffe in der innerfachlichen didaktischen Diskussion historical thinking (z.B. Kuhn, Weinstock \& Flaton, 1994; Lee \& Ashby, 2000; Seixas, 1996; Spoehr \& Spoehr, 1994; Wineburg, 1999) und Geschichtsbewusstsein (z.B. Jeismann, 1980; Körber, Schreiber et al., 2007; Pandel, 1987; von Borries, 1995), gefordert wird von Schülern also ein reflektierter und kritisch deutender Umgang mit verschiedensten historischen Ereignissen und Prozessen. Auf der konkreteren Ebene von Einzelkompetenzen sollen Schüler beispielsweise zur kritischen Arbeit mit historischen Quellen und Darstellungen befähigt werden. Sie sollen mit kontroversen Darstellungen umzugehen lernen und frühere Denkhorizonte und Handlungsspielräume bezogen auf spezifische historische Kontextbedingungen rekonstruieren. Dies sollen sie nicht ausschließlich im Rahmen des Geschichtsunterrichts tun, sondern das Gelernte ebenfalls auf die Begegnung mit Geschichte in ihrem Alltag übertragen. In dieser Form lassen sich die Anforderungen des Geschichtsunterrichts durchaus mit Kompetenzdefinitionen wie der von Weinert (2001) oder Klieme und Hartig (2007) ${ }^{1}$ in Einklang bringen und das Fach Geschichte müsste etwas überspitzt formuliert - geradezu prädestiniert für die Kompetenzforschung und die Umsetzung von Bildungsstandards sein. Dass diese Einschätzung jedoch keineswegs der Realität entspricht, ist auf zahlreiche Gründe zurückzuführen, von denen an dieser Stelle nur einige skizziert werden.

Ein erster Grund betrifft die Operationalisierbarkeit von Kompetenzen historischen Verstehens. Während sich für das Fach Mathematik der Umgang mit dem Zahlenraum von 1 bis 100 vergleichsweise leicht durch mathematische (Text-)aufgaben überprüfen lässt, ist beispielsweise die Kompetenz „eigene kritische Deutungen historischer Sachverhalte vornehmen und dabei Quellen und Darstellungen in angemessener Weise in die eigene Argumentation einbeziehen“ (Sauer, 2006, S. 12) weitaus schwieriger zu messen. Dies liegt sicherlich zum einen an dem ausgesprochen hohen Abstraktionsgrad, die Kompetenzmodelle im geschichtsdidaktischen Diskurs aufweisen, zum anderen jedoch an der Komplexität des Gegenstandes „Geschichte“ selbst. Ein zweiter Grund ist ebenfalls mit dem Gegenstand „Geschichte“ verknüpft: die eine richtige Antwort gibt es bei der Beschäftigung mit Geschichte nur sehr selten. Im Geschichtsunterricht geht es idealtypisch - um den Umgang mit Quellen, die aufgrund einer bestimmten Perspektive des Verfassers entstanden sind bzw. um den Umgang mit kontroversen Darstellungen von Geschichte. Schüler sollen verstehen, dass es die Geschichte nicht gibt, sondern

\footnotetext{
${ }^{1}$ Die Kompetenzdefinitionen werden in Abschnitt 1.1 näher erläutert.
} 
dass wir es ständig mit Rekonstruktionen von historischen Begebenheiten zu tun haben (z.B. Schreiber et al., 2007; Martens, 2008). Dieser Anspruch steht jedoch einer ökonomischen, quantitativen Messung von Kompetenzen historischen Verstehens im Wege; in vielen Fällen müsste ein solcher Anspruch durch eine prozessorientierte Erfassung verfolgt werden (Wie kommt ein Schüler zu einer bestimmten Argumentation? Kann er seine Position begründen und verteidigen?) (von Borries, 2007).

Ein dritter Grund für die bisher fehlende Messung von Kompetenzen historischen Verstehens liegt in der Forschungstradition des Faches begründet. Dem Gegenstand Geschichte angemessen arbeitet die Geschichtswissenschaft nur selten mit empirischen oder gar experimentellen Forschungsmethoden. Ein historisches Ereignis lässt sich nicht im Nachhinein experimentell manipulieren, Störvariablen können nicht ausgeschlossen werden und auch die Auswirkungen eines historischen Ereignisses können nicht separat voneinander und unabhängig vom historischen Kontext betrachtet werden (z.B. Lee, Dickinson \& Ashby, 1998). Vielmehr wird Geschichtswissenschaft durch die Arbeit mit verschiedensten Quellen und Darstellungen, das Aufstellen plausibler Deutungen und die Entwicklung schlüssiger und triftiger Interpretationen betrieben. Aufgrund dieser das Fach konstituierenden Arbeits- und Denktradition ist der Weg zu einer (meist) empirisch quantitativen Kompetenzforschung in der Didaktik dieses Faches ein weiterer als dies beispielsweise in den Naturwissenschaften der Fall ist. Wobei das nicht bedeutet, dass empirische Forschung in der Geschichtsdidaktik ein gänzlich unbeschriebenes Blatt wäre (z.B. Gautschi, Moser, Reusser \& Wiher, 2007; Günther-Arndt \& Sauer, 2006; Hodel \& Ziegler, im Druck; von Borries, 1994, 1995; Voss \& Wiley, 1997; Wiley \& Voss, 1999; Wineburg, 1991).

Die Kompetenz- und Bildungsstandardsdebatte trifft also auf ganz spezifische inhaltliche und methodische Herausforderungen für das Fach Geschichte und seine Didaktik. An diesem Punkt setzt das Projekt „Kompetenzstufen historischen Verstehens“ im DFG-geförderten Graduiertenkolleg „Passungsverhältnisse schulischen Lernens Verstehen und Optimieren“ an der Universität Göttingen an. Im interdisziplinären Diskurs zwischen pädagogischer Psychologie und Geschichtsdidaktik sollen Dissertationen entstehen, die historische Kompetenzen einer empirischen Erfassung zugänglich machen. Ein erster Schritt ist dabei die Auswahl eines Kompetenzbereiches historischen Verstehens, der sich für eine interdisziplinäre Betrachtung eignet.

Das Konzept der Perspektivenübernahme ermöglicht eine solche Betrachtung in vielerlei Hinsicht. In der geschichtsdidaktischen Kompetenzdiskussion - sowohl in 
Deutschland als auch international - wird Perspektivenübernahme als integraler Bestandteil historischen Verstehens und Lernens angesehen (Kapitel 1). In der Psychologie wird Perspektivenübernahme bzw. -koordination als Voraussetzung für die Bewältigung sozialer Interaktionen verstanden. Mit der Theorie von Robert L. Selman (Abschnitt 2.2) liegt ein Entwicklungsstufenmodell vor, welches die psychologische Grundlage der vorliegenden Arbeit darstellt. Zusammen mit weiteren psychologischen Ansätzen, die die Rolle von Kontextfaktoren für die Kompetenz zur Perspektivenübernahme betonen (Abschnitt 2.3), bietet das Modell zahlreiche Anknüpfungspunkte für eine empirische Erfassung von Perspektivenübernahme im Kontext Geschichte (Abschnitt 2.4). Aus diesem interdisziplinären Kontext werden Forschungsziele zur Erfassung von Perspektivenübernahme als Kompetenz historischen Verstehens abgeleitet (Kapitel 3) und in Form von drei Publikationen verfolgt (Kapitel 4 und Kapitel 5). Abschließend werden die theoretischen und empirischen Erträge dieser Arbeit zusammengefasst und ihre Implikationen für die Messung von Kompetenzen historischen Verstehens diskutiert (Kapitel 6). 


\section{Perspektivenübernahme als Bestandteil von Kompetenzmodellen historischen Verstehens}

\subsection{Vorbemerkungen}

Im ersten Kapitel dieser Arbeit werden geschichtsdidaktische Kompetenzmodelle skizziert, die Perspektivenübernahme als einen zentralen Bestandteil historischen Verstehens ansehen. Dafür ist zunächst die Klärung zweier zentraler Begriffe notwendig: Kompetenz und Modell.

Der Begriff Kompetenz ist in den letzten Jahren zum Modewort der Bildungsforschung avanciert. Ursprünglich geht er auf Arbeiten von Chomsky (z.B. 1968) zur Sprachkompetenz und zu Theorien des kommunikativen Handelns zurück. In dieser Tradition wird Kompetenz als ein generatives, situationsunabhängiges kognitives System verstanden und von der Performanz - der situationsabhängigen Realisierung der Kompetenz - abgegrenzt. Demgegenüber wird Kompetenz in der Psychologie eher funktional-pragmatisch verstanden als die Fähigkeit einer Person, situativ geprägte Anforderungen zu bewältigen (Klieme \& Hartig, 2007). Weinert (2001) schließt in seiner vielzitierten Kompetenzdefinition sowohl Fähigkeiten und Fertigkeiten als auch motivationale, volitionale und soziale Bereitschaften ein, die es Individuen ermöglichen, Problemlösungen für variable Situationen zu entwickeln und umzusetzen. Für das Fach Geschichte weist Pandel (2005) darauf hin, dass der Begriff Kompetenz weitgehend alltagsweltlich gebraucht wird und oftmals nicht mehr meint als Können oder Fähigkeit. Für eine ausführliche Diskussion verschiedener Kompetenzkonzepte sei auf Kaufhold (2006) verwiesen. In der vorliegenden Arbeit orientiere ich mich an der Auffassung von Klieme und Hartig (2007), nach der Kompetenzen als ,erlernbare kontextspezifische Leistungsdispositionen“ verstanden werden, ,die sich funktional auf Situationen und Anforderungen in bestimmten Domänen beziehen“ (S. 17).

Der Begriff Modell wird wissenschaftstheoretisch als eine vereinfachende Beschreibung der Wirklichkeit verstanden. Die fachdidaktischen Modelle, die in dieser Arbeit skizziert werden, geben jedoch (mit Ausnahme von Abschnitt 1.6) nicht vor, die Wirklichkeit zu beschreiben. Es handelt sich vielmehr um normative Vorstellungen von Kompetenzen, die Schüler im Geschichtsunterricht erwerben sollen. Empirische Belege für die Struktur historischer Kompetenzen oder deren Ausprägungen in verschiedenen 
Alters- bzw. Klassenstufen liegen bisher nur sehr vereinzelt vor und werden in den Modellen nicht systematisch integriert.

Das erste Kapitel beginnt mit einer Darstellung der drei einflussreichsten deutschsprachigen Kompetenzmodelle für das Fach Geschichte von Sauer (2006), Gautschi (2006) und der Kooperation FUER Geschichtsbewusstsein (Körber, Schreiber et al., 2007). Anschließend wird anhand von Bildungsstandards für Geschichte in England, in den USA und in Deutschland dargestellt, inwieweit die Kompetenz historische Perspektiven einzunehmen durch politische Vorgaben Eingang in den Geschichtsunterricht finden soll. Abschließend wird mit den Arbeiten von Ashby und Lee (1987; Lee \& Ashby, 2001) ein Modell vorgestellt, welches ausschließlich die Kompetenz zur historischen Perspektivenübernahme thematisiert und auf der Basis einer qualitativen Studie erste Entwicklungsperspektiven aufzeigt. Im Anschluss an die Darstellung der Kompetenzmodelle wird eine Bewertung für die Nutzbarkeit im Hinblick auf die empirische Erfassung von historischer Perspektivenübernahme vorgenommen.

Der Zugang zu den fachdidaktischen Kompetenzmodellen wird dadurch erschwert, dass für die Fähigkeit, historische Perspektiven einzunehmen, sehr unterschiedliche Begriffe verwendet werden. Auf diese theoretische Unschärfe wurde in der Literatur bereits mehrfach hingewiesen (z.B. Baring, 2004; Foster, 2001; Lee \& Ashby, 2001). Da, wie ich auf den folgenden Seiten darlegen werde, das Einnehmen historischer Perspektiven vornehmlich als eine kognitive Kompetenz verstanden wird, und nur am Rande mit Konzepten wie Empathie, Sympathie, Mitgefühl oder Identifikation in Verbindung gebracht wird, verwende ich für meine eigene Untersuchung den in der Psychologie üblichen Begriff Perspektivenübernahme. Bei der Darstellung der geschichtsdidaktischen Kompetenzmodelle übernehme ich jedoch die von den jeweiligen Autoren verwendeten Begrifflichkeiten.

\subsection{Mit Perspektivität in der Geschichte umgehen und Fremdverstehen leisten im Kompetenzmodell von Michael Sauer}

Das Kompetenzmodell für den Geschichtsunterricht (Sauer, 2006) besteht aus drei Bereichen: Sachkompetenz, Deutungs- und Reflexionskompetenz sowie MedienMethoden-Kompetenz. Diesen Bereichen werden Teilkompetenzen zugeordnet; der Schwerpunkt liegt dabei auf der Deutungs- und Reflexionskompetenz. In diesem Be- 
reich lassen sich auch Annahmen zur Perspektivenübernahme verorten: Mit Perspektivität in der Geschichte umgehen und Fremdverstehen leisten werden von Sauer als zwei Teilkompetenzen konzipiert und jeweils in drei Unterkompetenzen gegliedert, die sie genauer charakterisieren und erste Vorschläge zu Lernprogressionen machen sollen.

Die Unterkompetenzen zu Mit Perspektivität in der Geschichte umgehen erscheinen relativ heterogen. Schüler sollen zunächst Perspektivität in Quellen erkennen und analysieren können. Sie sollen sich weiterhin in unterschiedliche Perspektiven hineinversetzen können und in ihnen sprechen und handeln können. Drittens sollen sie historische Situationen und Ereignisse parallel aus unterschiedlichen Perspektiven betrachten können.

Mit der ersten Unterkompetenz ist die Fähigkeit von Schülern angesprochen, die mit methodischen Aspekten des historischen Lernens zusammenhängt, insbesondere mit der Quellenarbeit. Dafür spricht auch, dass diese Kompetenz mit annähernd gleichem Wortlaut noch einmal unter dem Kompetenzbereich Medien-Methoden-Kompetenz aufgeführt ist. Die zweite Unterkompetenz beschreibt das Hineinversetzen in unterschiedliche Perspektiven und beinhaltet mit der Forderung nach „,sprechen und handeln“ eine aktive Auseinandersetzung mit historischen Personen, beispielsweise im Rollenspiel. Die dritte Unterkompetenz verlangt, historische Ereignisse auf einer Metaebene gleichzeitig aus unterschiedlichen Perspektiven zu betrachten. Offen bleibt, welche Perspektiven hier gemeint sind - sind es verschiedene Rollen von Akteuren, die am gleichen historischen Ereignis beteiligt waren, ist es die heutige Sichtweise verglichen mit der aus mehr oder weniger fernen Vergangenheiten, sind es Perspektiven von Historikern, die unterschiedliche Deutungen vornehmen oder ist alles zugleich impliziert? Was die Koordination verschiedener Perspektiven betrifft, nimmt Sauer zwischen der zweiten und dritten Unterkompetenz einen qualitativen Sprung an, der sich auch in Selmans Modell zur sozialen Perspektivenübernahme findet (Selman, 1980, vgl. Abschnitt 2.2): Während es bei der zweiten Unterkompetenz auf ein zwischenzeitliches Verlassen der eigenen Perspektive anzukommen scheint, fordert die dritte Unterkompetenz eine Integration der eigenen mit verschiedenen anderen Perspektiven sowie deren Koordination.

Die Teilkompetenz Fremdverstehen leisten ist ebenfalls durch drei Unterkompetenzen charakterisiert. Schüler sollen historische Verhältnisse und das Handeln von Menschen aus vergangenen Zeiten unvoreingenommen als anders (und nicht als rückständig) wahrnehmen. Darüber hinaus sollen sie das Handeln von Menschen früher auf der Basis der zeitgenössischen Wertvorstellungen, Rahmenbedingungen und Hand- 
lungsspielräume wahrnehmen. Letztlich sollen sie gegenwärtige und historische Wertvorstellungen und Urteilsnormen kritisch aufeinander beziehen.

Sauer thematisiert mit Fremdverstehen leisten also die Begegnung der fremden (historischen) Welt mit der heutigen (Schüler-) Sichtweise. Es geht also um das Fremde und das Eigene und die Erklärung von Unterschieden zwischen beiden. Während die erste Unterkompetenz darin besteht anzuerkennen, dass Menschen früher anders waren, geht die zweite Unterkompetenz darüber hinaus. Sie verlangt eine historisch plausible Begründung, warum Menschen anders waren. Die dritte Unterkompetenz könnte man so interpretieren, dass das Verlassen der eigenen Perspektive möglich wird, um auch die eigene als eine von vielen möglichen Perspektiven zu analysieren und vergleichend mit der historischen Perspektive kritisch zu beleuchten.

Perspektivenübernahme wird in Sauers Kompetenzmodell durch die Verankerung in zwei Teilkompetenzen relativ viel Bedeutung beigemessen und somit als wichtiger Bestandteil historischen Denkens angesehen. $\mathrm{Zu}$ diskutieren bleibt, ob die Trennung in zwei separate Teilkompetenzen theoretisch gerechtfertigt ist. Auch wenn die Teilkompetenz Fremdverstehen eine dezidierte Urteils- und Wertkomponente enthält, lassen sich insbesondere bezüglich der jeweils dritten Unterkompetenzen große Parallelen feststellen. Mit der Formulierung der Unterkompetenzen liefert das Modell allenfalls erste Ansatzpunkte für Lernprogressionen bezogen auf Perspektivenübernahme als historische Kompetenz. Dies entspricht letztlich auch der Intention von Sauer (2006), ein erstes pragmatisches Modell als Basis für die Bildungsstandards des Verbandes der Geschichtslehrer (vgl. Abschnitt 1.5.3) zu entwerfen.

\subsection{Perspektivenübernahme als Bestandteil von Interpretationskompetenz, narra- tiver Kompetenz und Urteilskompetenz im Modell von Peter Gautschi}

Im Kompetenzmodell für den Geschichtsunterricht (Gautschi, 2006) werden fünf Kompetenzbereiche aufgeführt, die historisches Lernen im Schulkontext konstituieren. Diese Bereiche sind nicht als unabhängige Dimensionen aufzufassen, sondern als voneinander abhängend und sich gegenseitig unterstützend. Es handelt sich dabei um die Erschließungskompetenz, die Methodenkompetenz, die Interpretationskompetenz, die narrative Kompetenz und die Urteilskompetenz. Aussagen zur Perspektivenübernahme 
von Schülern finden sich in diesem Modell in den letzten drei Kompetenzbereichen wieder.

Interpretationskompetenz umschreibt den analytischen Umgang mit Geschichte. Dazu ist laut Gautschi (2006) das Hineinversetzen in Menschen aus der Vergangenheit notwendig und die Empfindung von Mitgefühl mit diesen Menschen. Weiterhin sollen Schüler Perspektiven identifizieren, die historischen Erzählungen und Erklärungen zugrunde liegen.

Bei der Betrachtung dieser Kompetenzen wird deutlich, dass von Gautschi weit mehr impliziert ist als nur Perspektivenübernahme. Lediglich das Hineinversetzen in Menschen aus der Vergangenheit ließe sich damit umschreiben. Die Empfindung von Mitgefühl tangiert vielmehr das Konstrukt der Empathie - über dessen Nutzen für historisches Lernen nebenbei bemerkt in der Geschichtsdidaktik keinesfalls Einigkeit herrscht (z.B. Foster, 2001). Mit der Identifikation von Perspektivität in historischen Erklärungen wird eine Kompetenz angesprochen, die Überschneidungen zum Bereich Methodenkompetenz aufweist.

Auch im Bereich narrative Kompetenz sind Überlegungen zur Perspektivenübernahme verankert. Schüler sollen einen Bezug zwischen vergangenen Phänomenen und der Gegenwart bzw. der eigenen Person herstellen. Perspektivenübernahme könnte dabei insofern eine Rolle spielen, als gegenwärtige und vergangene Vorstellungen und Handlungsweisen zueinander in Beziehung gesetzt werden. Damit hängt auch ein Bestandteil der Urteilskompetenz zusammen, nämlich die Analyse von Interessen und Werten, die bei den beteiligten Menschen für die Bewältigung von Situationen in der Vergangenheit eine Rolle gespielt haben. Dies erfordert von Schülern, historische Bedingungen sowie Denk- und Handlungshorizonte im Vergleich zu den heutigen Maßstäben zu rekonstruieren, um sich in historische Perspektiven hineinversetzen zu können.

\subsection{Kompetenz zur Reflexion und Erweiterung des Welt- und Fremdverstehens im} Kompetenz-Strukturmodell historischen Denkens der Gruppe FUER Geschichtsbewusstsein

Das Kompetenz-Strukturmodell historischen Denkens fußt auf der fundamentalen Annahme, dass der Ursprung der Geschichtswissenschaft das Bedürfnis des Men- 
schen ist, sich in der Zeit zu orientieren (Körber, Schreiber et al., 2007). Aufbauend auf der Disziplinären Matrix (Rüsen, 1983) und dem Prozessmodell historischen Denkens (Hasberg \& Körber, 2003) wurde ein Kompetenzmodell entwickelt, welches den Anspruch erhebt, über die schulische Geschichtsvermittlung hinauszureichen und für die gesamte Domäne des Umgangs mit Vergangenheit gültig zu sein (Schreiber et al., 2007).

Historisches Denken wird in diesem Modell als Funktion von vier unterscheidbaren Kompetenzbereichen dargestellt, die jedoch untereinander Zusammenhänge aufweisen können und sollen. Historische Fragekompetenzen, historische Methodenkompetenzen, historische Orientierungskompetenzen und historische Sachkompetenzen konstituieren so die Kompetenz, historisch zu denken.

Die Kompetenz historische Perspektiven einzunehmen lässt sich in diesem Modell als ein Teil der historischen Orientierungskompetenzen verorten. Historische Orientierungskompetenzen haben einen klar ausgewiesenen Gegenwartsbezug. Sie bündeln ,alle diejenigen Fähigkeiten, Fertigkeiten und Bereitschaften, die nötig sind, historisches Denken und seine Ergebnisse lebensweltlich wirksam werden zu lassen“ (Körber, Meyer-Hamme \& Schreiber, 2007, S. 473). Die Autoren unterteilen diesen Kompetenzbereich in vier Kernkompetenzen. Neben der Kompetenz zur Reorganisation des Geschichtsbewusstseins, der Kompetenz zur Identitätsreflexion und der Historischen Handlungsreflexionskompetenz formulieren sie die Kompetenz zur Reflexion und Erweiterung des Welt- und Fremdverstehens. Auf letztere wird an dieser Stelle näher eingegangen. Diese besteht in der Fähigkeit, Fertigkeit und Bereitschaft, die eigenen Vorstellungen von der Welt und ihren Menschen zu reflektieren. Eine solche Kompetenz erfordert das Überdenken historischer Ereignisse und Gegebenheiten und Prozesse im Verhältnis zu der eigenen (gegenwärtigen) Welt und Zeit. Damit sind Alteritätserfahrungen impliziert, also die Wahrnehmung und Verarbeitung zeitlicher, kultureller und intersubjektiver Differenzen (Schreiber, 2007). Aus dieser Definition spricht eine breit gefasste Konzeption dieser Kernkompetenz. Es geht um mehr als in einer konkreten Situation die Perspektive eines spezifischen historischen Akteurs einzunehmen, sondern vielmehr darum, Erkenntnisse, die aus der Beschäftigung mit historischen Fragestellungen gewonnen wurden, reflektiert auf die eigene Person und Lebenswelt zu beziehen.

Die Autoren machen weiterhin einen Versuch zur Unterscheidung von Niveaustufen dieser Kompetenz (Körber, Meyer-Hamme et al., 2007). Diese Abstufungen sind deduktiv aus einer für das gesamte Modell gültigen Graduierungslogik abgeleitet und 
bisher nicht empirisch untermauert. Auf dem Nullniveau ${ }^{2}$ ist der Mensch nicht in der Lage, sein Urteil über Welt und Menschen der Vergangenheit auf Basis neuer Erkenntnisse zu revidieren. Das basale Niveau der Kompetenz zum Fremd- und Weltverstehen ist dadurch gekennzeichnet, dass Menschen neu erworbene Informationen über die historische Welt als Einzelinformation erkennen. Veränderungen des eigenen Bildes der Vergangenheit bestehen allenfalls darin, Einzelinformationen unverbunden nebeneinander zu stellen ohne beispielsweise Gegensätze zwischen ihnen zu erkennen. Es folgt das intermediäre Niveau, welches durch konventionelle Operationen gekennzeichnet ist. Menschen übernehmen auf dieser Stufe gesellschaftlich-konventionelle Urteile für die Veränderung ihres Welt- und Fremdverstehens. Es können auch eigene Urteile erarbeitet werden, dies geschieht jedoch allenfalls angelehnt an stark konventionelle Vorstellungen. Das elaborierte und trans-konventionelle Niveau stellt die nächsthöhere Ausprägung dieser Kompetenz dar. Auf diesem Niveau werden alle gesellschaftlichen Deutungsangebote differenzierend reflektiert und die Person kommt auf Basis dessen und ihrer eigenen Perspektive zu einer eigenen Sicht. Für dieses Niveau sind Differenzierungsfähigkeit und Ambiguitätstoleranz unbedingte Voraussetzungen. Weiterhin wird ein Maximalniveau angenommen, welches aber wie das Nullniveau rein theoretischer Natur ist und nicht näher inhaltlich beschrieben wird.

Innerhalb des Modells werden Zusammenhänge dieser Kernkompetenz mit der Identitätsreflexion und der Handlungsreflexionskompetenz angenommen - eine weitere Betonung des Gegenwartsbezugs von Perspektivenübernahme. Es werden ebenfalls Zusammenhänge mit der historischen Fragekompetenz, der Sachkompetenz und der Methodenkompetenz vermutet.

Auch über die Zusammenhänge innerhalb des Modells hinaus erscheint das Modell anschlussfähig für eine interdisziplinäre Betrachtung von Perspektivenübernahme. Die Annahmen, die in diesem Modell zu den Bestandteilen der Kompetenz zur Reflexion und Erweiterung des Welt- und Fremdverstehens gemacht werden, weisen - trotz zentraler Unterschiede im Gegenstandsbereich - Parallelen zur Theorie sozialer Perspektivenkoordination nach Selman (1980) auf. In Abschnitt 2.4 wird darauf ausführlicher eingegangen.

\footnotetext{
${ }^{2}$ Dieses Niveau wird von den Autoren als „fiktiv“ beschrieben, es werden demnach keine realen Fälle erwartet, die diesem Niveau entsprechen. Es dient lediglich als Ankerpunkt der Graduierungslogik.
} 


\subsection{Perspektivenübernahme als Bestandteil von Bildungsstandards für das Fach Geschichte}

\subsubsection{Perspektivenübernahme als Teil des National Curriculum for History in England}

Die Diskussion um Schülerkompetenzen und Standards findet in England bereits seit 1988 in Form des National Curriculum Eingang in den Schulalltag. ${ }^{3}$ In drei sogenannten key stages wird festgeschrieben, welches Wissen, welches Verständnis und welche Fähigkeiten Schüler im Alter von 7, 11 und 14 Jahren im Fach Geschichte entwickeln sollen. Zusätzlich liegen für jede key stage Standards vor, die ein Großteil der Schüler erreichen soll. Diese Standards lassen sich in neun Levels unterteilen.

Der Fokus des National Curriculum für das Fach Geschichte (Department for Education and Employment \& Qualifications and Curriculum Authority, 1999) liegt auf fünf Aspekten historischen Lernens, die bei Schülern im Unterricht gefördert werden sollen. Es handelt sich dabei um chronologisches Verstehen, Wissen über und Verständnis von Ereignissen, Personen und Veränderungen in der Vergangenheit, historische Interpretation, historische Untersuchung sowie Organisation und Kommunikation. ${ }^{4}$ Hinzu kommen für jede key stage bestimmte historische Inhalte, die in lokale, britische, europäische und Weltgeschichte unterteilt sind und anhand derer die oben genannten fünf Aspekte im Unterricht vermittelt werden sollen. Für die Analyse in der vorliegenden Arbeit werden die historischen Inhalte jedoch nicht weiter beachtet.

Die Forderung nach der Fähigkeit, historische Perspektiven einzunehmen, ist in den Aspekten chronologisches Verstehen sowie Wissen über und Verständnis von Ereignissen, Personen und Veränderungen in der Vergangenheit verankert. Auf key stage 1 (im Alter von sieben Jahren) sollen Schüler lernen, wie sich die Vergangenheit von der Gegenwart unterscheidet. Dazu sollen sie erlernen, warum Menschen in der Vergangenheit in bestimmter Weise gehandelt haben. Zudem sollen sie Unterschiede zwischen verschiedenen Lebensweisen zu unterschiedlichen Zeiten identifizieren. Auf key stage 2 (im Alter von 11 Jahren) wird von Schülern gefordert, charakteristische Merkmale bestimmter Epochen und Gesellschaftsformen zu erkennen. Schüler sollen Ideen,

\footnotetext{
${ }^{3}$ In der vorliegenden Arbeit dient die Fassung des National Curriculum von 1999 als Grundlage, die bis 2011 Gültigkeit hat. Ab September 2008 wird mit der Implementierung einer weiterentwickelten Fassung begonnen.

${ }^{4}$ im Original: chronological understanding, knowledge and understanding of events, people and changes in the past, historical interpretation, historical enquiry, organisation and communication.
} 
Vorstellungen, Einstellungen und Erfahrungen von Männern, Frauen und Kindern in der Vergangenheit kennenlernen. Weiterhin sollen sie soziale, kulturelle, religiöse und ethnische Unterschiede in den im Unterricht behandelten Gesellschaften thematisieren. Die Forderungen für key stage 3 (im Alter von 14 Jahren) sind denen der zweiten Stufe sehr ähnlich. Der einzige Unterschied ist, dass auf dieser höheren Stufe zusätzlich die vergleichende Beschreibung und Analyse solcher charakteristischen Epochen- und Gesellschaftsmerkmale verlangt wird.

Die Ausdifferenzierung dieser Fähigkeitsanforderungen in Niveaustufen findet im National Curriculum in Form der neun Levels statt. Für die Lernprogression bezogen auf Perspektivenübernahme liefern diese Levels jedoch nur geringen Erkenntnisgewinn. Die kurzen Levelbeschreibungen (ca. fünf bis sechs Sätze) umfassen jeweils alle fünf Aspekte historischen Lernens und bleiben sehr vage. Formulierungen aus den Beschreibungen der key stages werden größtenteils wortwörtlich übernommen und allenfalls etwas abgestuft (,recognition of some of the similarities and differences...“, ,beginning to recognise...“, ,,analyse links between...“) (Department for Education and Employment \& Qualifications and Curriculum Authority, 1999, S. 39-40).

Nach Ansicht der Autoren des National Curriculum kann der Geschichtsunterricht zusätzlich zu den fachspezifischen Fähigkeiten auch die moralische, soziale und kulturelle Entwicklung der Schüler begünstigen. Bestandteile von Perspektivenübernahme werden als überfachliche Fähigkeiten mehrfach angesprochen. So sollen Schüler entdecken, wie unterschiedliche Interpretationen der Vergangenheit verschiedene Standpunkte und Wertvorstellungen reflektieren. Weiterhin sollen sie verstehen, wie unterschiedliche Gesellschaftsformen und politische Systeme in der Vergangenheit organisiert waren. Letztlich sollen sie Unterschiede und Gemeinsamkeiten zwischen verschiedenen Kulturen und innerhalb einer Kultur erkennen. Diese Forderungen spiegeln eine Auffassung von Perspektivenübernahme wider, die sowohl Bezüge zum historischen als auch zum sozialen, moralischen und kulturellen Verstehen einschließt. Es erscheint aufgrund dieser Überlegungen plausibel, dass Perspektivenübernahme eine Vermittlungsrolle zwischen fachspezifischen und fachübergreifenden Kompetenzen einnehmen könnte. 
Ähnlich wie in England wird die Debatte um Standards im Bildungswesen in den USA bereits seit mehreren Jahrzehnten geführt. Seit Ende der 1980er Jahre wurde die Einführung nationaler Standards diskutiert und 1994 mit dem Educate America Act von der Clinton-Regierung festgeschrieben. Das Fach Geschichte war gleich zu Beginn von der Einführung des neuen Gesetzes betroffen. Unter der Federführung des National Council for History Standards wurden in einem Konsortium aus verschiedensten akademischen und schulnahen Experten für das Fach Geschichte die National Standards for United States History entwickelt (National Center for History in the Schools, 1996).

Kernstück der Standards für Geschichte sind fünf historical thinking skills. Es handelt sich dabei um chronologisches Denken, historisches Verständnis, historische Analyse und Interpretation, historische Forschungsmethoden sowie historische Fallanalyse und Entscheidungsfindung. ${ }^{5}$ Hinzu kommen historische Inhalte (als historical understanding bezeichnet) anhand derer die Standards erlernt werden sollen.

Elemente von Perspektivenübernahme lassen sich insbesondere in den Standards zwei und drei verorten. In Standard zwei (historisches Verständnis) wird unter anderem gefordert, dass Schüler historische Narrationen unter Einbezug der eigenen Vorstellungskraft lesen. Dazu gehört, dass sie den historischen Kontext eines Ereignisses berücksichtigen und Werte, Perspektiven, Handlungsoptionen und Möglichkeiten identifizieren, die den jeweiligen Ort und die Zeit charakterisierten. Implizite Motive, Hoffnungen, Ängste, Stärken und Schwächen der historischen Akteure sollen anhand der Narrationen rekonstruiert werden. Weiterhin sollen Schüler historische Perspektiven aufzeigen, indem sie Vergangenheit unter Berücksichtigung der ihr eigenen Bedingungen beschreiben und sie mit den Augen der beteiligten historischen Akteure sehen. Dies sollen sich die Schüler durch Literatur, Tagebücher, Briefe etc. erschließen. Damit sollen sie gleichzeitig vermeiden, die Vergangenheit ausschließlich anhand heutiger Normen und Werte zu beurteilen (present-mindedness).

Auch für Standard drei (historische Analyse und Interpretation) ist die Fähigkeit, vergangene Perspektiven einzunehmen, notwendig. So sollen Schüler unterschiedliche Ideen, Werte, Persönlichkeiten, Verhaltensweisen und Institutionen vergleichend betrachten und kontrastieren. Sie sollen außerdem multiple Perspektiven von Völkern der

\footnotetext{
5 im Original: chronological thinking, historical comprehension, historical analysis and interpretation, historical research, historical issues-analysis and decision-making.
} 
Vergangenheit berücksichtigen, indem sie deren unterschiedliche Motive, Vorstellungen, Interessen, Hoffnungen und Ängste herausstellen. Drittens wird in Standard drei Perspektivenübernahme als Metakompetenz in Bezug auf den Vergleich gegensätzlicher historischer Narrationen gefordert. Zugänge verschiedener Historiker, die unterschiedliche Fragen an die Geschichte stellen und unterschiedliche Quellen heranziehen, sollen verglichen werden. Dabei sollen Schüler die Erfahrungen, Perspektiven, Vorstellungen und Sichtweisen berücksichtigen, die zu solchen unterschiedlichen Erklärungsansätzen geführt haben.

$\mathrm{Zu}$ Niveaustufen dieser Kompetenzen werden in den Standards keine Aussagen gemacht. Anhand aller vorgesehenen historischen Inhalte zur US-amerikanischen Geschichte wird jedoch für jeden aufgestellten Standard ausdifferenziert, welche Arbeitsaufträge Schüler erfüllen sollen um ihre Kompetenzen zu demonstrieren. Dabei wird jeweils unterschieden zwischen Aufgaben für die Klassenstufen fünf/sechs, sieben/acht und neun bis zwölf. Es liegen keine Informationen dazu vor, ob diese Arbeitsaufträge tatsächlich zur Förderung von Perspektivenübernahme geeignet sind. Auch Kriterien für die Einstufung von Schülerleistungen sind nicht vorhanden.

Ähnlich wie in England wird auch in den US-Standards der Gegenwartsbezug von Geschichte im Allgemeinen und Perspektivenübernahme im Besonderen betont. Es wird postuliert, dass das Fach Geschichte Schülern die Möglichkeit eröffnet, ein umfassendes Verständnis der Welt zu entwickeln und verschiedene Kulturen und Lebensweisen, die sich von der eigenen unterscheiden, zu verstehen.

\subsubsection{Perspektivenübernahme als Teil von Bildungsstandards für das Fach Geschichte in Deutschland}

In Deutschland existieren im Gegensatz zu England und den USA bisher keine einheitlichen Bildungsstandards für das Schulfach Geschichte. Für jedes Bundesland und jede Schulform liegen unterschiedliche Rahmenrichtlinien, curriculare Vorgaben, Bildungspläne etc. vor, die beschreiben, welche Kompetenzen und welche Sachinhalte Schüler im Unterricht erwerben sollen. Die Kompetenz, historische Perspektiven einzunehmen, wird in diesen Vorgaben an verschiedenen Stellen betont. In den Leitgedanken zum Kompetenzerwerb für das Fach Geschichte des Landes Baden-Württemberg wird von Schülern der Klassenstufen sieben und acht beispielsweise gefordert, dass Schüler 
„,interessengebundene Verhaltensweisen der handelnden Personen“ erkennen (Kultusministerium Baden-Württemberg, 2004, S. 218). Dies sei insbesondere wichtig, da es das Bewusstsein für das eigene Handeln oder Nichthandeln schärfe und die Kritikfähigkeit und Einsicht in moralische Maßstäbe fördere. Auch in den curricularen Vorgaben des Landes Niedersachsen (Niedersächsisches Kultusministerium, 2004) wird historische Perspektivenübernahme im Hinblick auf soziale Kompetenzen in der Gegenwart als notwendiger Bestandteil des Faches Geschichte angesehen. Die Schüler sollen sich mit Wertvorstellungen und Handlungsmustern auseinandersetzen und diese in ihrer eigenen zeitlichen Berechtigung verstehen. Diese Reflexion gehöre zu den Voraussetzungen für die eigene Identitätsfindung und die Entwicklung von Toleranz. Auf welche Weise diese Kompetenzen mit den historischen Inhalten verknüpft werden sollen, die für die entsprechenden Klassenstufen im Fach Geschichte vorgesehen sind, wird von den Kultusministerien dieser beiden Bundesländer nicht näher ausgeführt. Die Rahmenlehrpläne Geschichte von Berlin (Senatsverwaltung für Bildung, Jugend und Sport Berlin, 2006a; 2006b) werden diesbezüglich deutlicher. Von Schülern wird verlangt, sowohl ihre eigene als auch gesellschaftliche Perspektiven in der Beschäftigung mit Vergangenheit zunehmend sachgerecht einzuschätzen. Perspektivenübernahme findet als Bestandteil von Deutungs- und Analysekompetenz Eingang in die Rahmenlehrpläne für die Sekundarstufen eins und zwei. So sollen die Schüler beispielsweise die Handlungen von historischen Akteuren unter dem Aspekt zeitbedingter Handlungsspielräume und Zwangslagen untersuchen. Auch unterschiedliche Gruppenperspektiven sollen bezogen auf ihre Standortgebundenheit analysiert werden. Diese Kompetenzanforderungen werden auf verschiedene historische Inhalte beispielhaft angewendet und für die Sekundarstufe eins zudem in einen Haupt-, einen Real- sowie einen Gymnasialzweig unterteilt. Weiterhin wird Perspektivenübernahme als Bestandteil von Urteilsund Orientierungskompetenz angesehen. Schüler sollen historische Perspektiven beschreiben und beurteilen, ,indem sie das Handeln historischer Akteure im Kontext ihrer zeitgenössischen Wertvorstellungen wahrnehmen, analysieren und ansatzweise gewichten“" (Senatsverwaltung für Bildung, Jugend und Sport Berlin, 2006a, S. 16). Auch die Bedeutung, die dem Fach Geschichte und der Kompetenz zur Perspektivenübernahme für die gegenwärtige Lebenswelt der Schüler beigemessen wird, wird an vielen Stellen des Rahmenlehrplans deutlich. So heißt es beispielsweise: „Die Schülerinnen und Schüler (...) untersuchen und reflektieren die Vielfalt der Möglichkeiten menschlichen Han- 
delns in der Vergangenheit und entwickeln daraus Konsequenzen für die Gegenwart“" (Senatsverwaltung für Bildung, Jugend und Sport Berlin, 2006a, S. 16).

Zwei Versuche, Kompetenzanforderungen für das Fach Geschichte bundeseinheitlich festzuschreiben, sollen an dieser Stelle erwähnt werden. Zum einen sind dies die Einheitlichen Prüfungsanforderungen in der Abiturprüfung Geschichte (EPA), die von der Kultusministerkonferenz 2005 beschlossen wurden, zum anderen die Bildungsstandards Geschichte für das Gymnasium, 2006 veröffentlicht vom Verband der Geschichtslehrer Deutschlands. In beiden Dokumenten wird Perspektivenübernahme als ein wichtiger Bestandteil historischer Kompetenz angesehen.

Die EPA Geschichte (Kultusministerkonferenz, 2005) fordern von Schülern, die die Abiturprüfung absolvieren, die Fähigkeit zur sinnbildenden Darstellung von Geschichte. Diese ermögliche es ihnen, am öffentlichen Diskurs über Geschichte teilzunehmen. Dazu werden drei Teilkompetenzen benötigt: Sach-, Methoden- und Urteilskompetenz. Perspektivenübernahme ist als Teil der Urteilskompetenz zu erkennen. So sollen Schüler ethische, moralische und normative Kategorien auf historische Sachverhalte anwenden, indem sie deren Zeitbedingtheit und Dauerhaftigkeit mitberücksichtigen. Es gilt also, moralische Vorstellungen bei der Begegnung mit Geschichte einzubeziehen, diese jedoch vor dem Hintergrund der damals herrschenden Bedingungen zu reflektieren.

Als Basis für die Bildungsstandards Geschichte (Verband der Geschichtslehrer Deutschlands, 2006) dient das Kompetenzmodell von Sauer (2006) (siehe Abschnitt 1.2). Ähnlich wie in den National Standards for United States History (National Center for History in the Schools, 1996) wird in den Bildungsstandards Geschichte eine Verknüpfung von Kompetenzen und Sachwissen hergestellt. So bedeutet das Hineinversetzen in verschiedene Perspektiven für Schüler der fünften und sechsten Klasse, beispielsweise Ständekämpfe in Rom aus der Perspektive der Patrizier oder der Plebejer nachzuvollziehen. Schüler der Klassen sieben und acht müssen, wenn sie Handlungen von Menschen auf Basis der zeitgenössischen Wertvorstellungen wahrnehmen sollen, die Beschränkung der Bildungsmöglichkeiten im hohen Mittelalter auf Adel, Klöster und reiche Stadtbürger als gegeben voraussetzen. 


\subsection{Historical Empathy im Modell von Rosalyn Ashby und Peter Lee}

In der Diskussion über Kompetenzen historischen Denkens ist vielfach von second-order concepts die Rede. Im Gegensatz zu historischen Begriffen oder Konzepten erster Ordnung wie König, Staat oder Revolution zielen solche Konzepte zweiter Ordnung auf grundlegende epistemologische Einsichten in die Domäne Geschichte und stellen eine Meta-Ebene für die Beschäftigung mit Geschichte dar (Lee et al., 1998). Das Verständnis von Kontinuität und Wandel, Ursache und Wirkung oder der Umgang mit diversen historischen Quellen gelten als Konzepte zweiter Ordnung. Auch historical empathy ist dieser Kategorie zuzuordnen.

Das Konzept historical empathy wurde von britischen Forschern hauptsächlich wegen seiner Griffigkeit eingeführt und Begriffen wie rational understanding oder perspective taking vorgezogen (Lee \& Ashby, 2001). Wenngleich dieses Konzept in der britischen Diskussion um historische Kompetenzen als gut implementiert gelten kann, birgt es diverse Schwierigkeiten in der Interpretation, mit denen sich die Autoren selbst kritisch auseinandersetzen (z.B. Ashby \& Lee, 1987; Lee \& Ashby, 2001). So werde empathy oftmals im Sinne von Identifikation oder Sympathie mit historischen Akteuren oder als Ausdruck eines Gefühls verstanden. Verstärkt wird dieser Eindruck einer konzeptionellen Unschärfe durch psychologische Definitionen, die Empathie als Tendenz bezeichnen, Gefühle von Wärme und Responsivität in Bezug auf andere Personen zu empfinden (z.B. Davis, 1983a).

Die Autoren definieren historical empathy jedoch klar als eine intellektuelle Leistung - trotz des Festhaltens an dem eher emotional konnotierten Begriff:

Empathy, as historical understanding, demands hard thinking on the basis of evidence. It requires students to know some history, and to be able to use that knowledge in order to explain actions and institutions. If it is to be given any sensible meaning in history, empathy is where you get when you have done the hard thinking, and produced an explanation based on the evidence you can find. It means entertaining complex ideas and seeing how they shape views of historical circumstances and goals, even when such ideas and goals may be very different from (and perhaps opposed to) our own (Lee \& Ashby, 2001, p. 25).

An dieser Definition ist erkennbar, dass das Konstrukt historical empathy in den Prozess historischer Erkenntnisgewinnung, insbesondere in die Auswertung von Belegen, z.B. in Form von historischen Quellen, eingebettet ist. Inhaltlich betonen die Autoren die Notwendigkeit, Ideen und Zielvorstellungen historischer Akteure in die Erklä- 
rung vergangener Situationen einzubeziehen, auch wenn diese im Gegensatz zu heutigen Vorstellungen stehen.

Neben einer theoretischen Konzeption liefern Ashby und Lee (1987) Befunde aus qualitativen Studien um das Konstrukt historical empathy empirisch auszudifferenzieren. Sie führten Gruppendiskussionen (ohne Versuchsleiter) mit Schülern zwischen 11 und 18 Jahren an einer Gesamtschule in der Nähe Londons durch. Aus diesen Ergebnissen leiten sie ein Modell für historical empathy ab, welches eine Progression beschreibt. Die Autoren stellen ihr Modell als eine Abfolge vor, die dadurch gekennzeichnet ist, dass auf den unteren Levels bestimmte Schwierigkeiten und Widersprüche bei der Interpretation historischer Situationen auftreten, die durch das nächsthöhere Level aufgelöst werden können. Sie interpretieren das Modell jedoch nicht im Sinne von Entwicklungsstufen, wie sie beispielsweise für die kognitive Entwicklung von Piaget entwickelt wurden. Sie betonen vielmehr mögliche Kontexteinflüsse, die dazu führen können, dass Schüler sich auf einem höheren Level befinden können, wenn sie sich mit historischen Themen beschäftigen, die ihnen bereits vertraut sind. Weiterhin weisen die Autoren auf die Instabilität der Levels hin. Bei intensiver Beschäftigung mit einem spezifischen historischen Problem könne ein Schüler auf ein geringeres Level zurückfallen oder höhere Levels erreichen. In Tabelle 1 sind die Levelbeschreibungen zusammengefasst. Auszüge aus den Gruppendiskussionen zur Illustration jedes Levels finden sich bei Ashby und Lee (1987). 
Tabelle 1: Zusammenfassung des Modells für Historical Empathy (vgl. Ashby \& Lee, 1987)

\begin{tabular}{|c|c|}
\hline Level & Kurzbeschreibung \\
\hline 1 The „Divi“ Past & $\begin{array}{l}\text { Historische Handlungen und Institutionen sind per } \\
\text { se unverständlich. } \\
\text { Menschen in der Vergangenheit werden als dumm } \\
\text { wahrgenommen, da es ihnen an „besseren“ Hand- } \\
\text { lungsalternativen mangelte, die für Menschen heute } \\
\text { offensichtlich sind. }\end{array}$ \\
\hline 2 Generalized Stereotypes & $\begin{array}{l}\text { Stereotype Vorstellungen über Funktionen und Rol- } \\
\text { len von Menschen werden zur Erklärung von } \\
\text { „rückständigen“ Handlungen herangezogen. }\end{array}$ \\
\hline 3 Everyday Empathy & $\begin{array}{l}\text { Die Situation von Menschen in der Vergangenheit } \\
\text { wird ansatzweise berücksichtigt. Ausgangspunkt } \\
\text { sind dabei heutige Vorstellungen, die auf die Ver- } \\
\text { gangenheit übertragen werden. Handlungsalternati- } \\
\text { ven werden auf Basis heutiger Vorstellungen ent- } \\
\text { wickelt. }\end{array}$ \\
\hline $\begin{array}{l}4 \text { Restricted Historical Em- } \\
\text { pathy }\end{array}$ & $\begin{array}{l}\text { Unterschiede zwischen Gegenwart und der spezifi- } \\
\text { schen historischen Situation werden erkannt und } \\
\text { analysiert. Die Analyse bleibt auf die Einzelsitua- } \\
\text { tion beschränkt und nimmt noch nicht den breiteren } \\
\text { historischen Kontext in den Blick. }\end{array}$ \\
\hline $\begin{array}{l}5 \text { Contextual Historical Em- } \\
\text { pathy }\end{array}$ & $\begin{array}{l}\text { Klare Differenzierung zwischen dem historisch } \\
\text { Handelnden und einem Historiker, zwischen Ge- } \\
\text { genwart und Vergangenheit. Vorstellungen, Werte, } \\
\text { Ziele von früher werden unter Einbeziehung des } \\
\text { historischen Kontextes rekonstruiert und von heuti- } \\
\text { gen unterschieden. Implizite Motive oder versteckte } \\
\text { Funktionen werden zur Erklärung von Handlungen } \\
\text { in der Vergangenheit vorgeschlagen. }\end{array}$ \\
\hline
\end{tabular}

Die Autoren betrachten das Modell als vorläufig und tentativ, weisen aber darauf hin, dass ihre Progressionsannahmen für dieses Konstrukt im Einklang mit Befunden aus anderen Studien stehen (z.B. Shemilt, 1980). Eine Validierung des Modells steht jedoch bisher noch aus. Zudem lassen sich die aufgestellten Levels aufgrund der Methode Gruppendiskussion nicht ohne Weiteres auf die Entwicklung individueller Kompetenzausprägungen übertragen. Denn die Schüleraussagen, die in das Modell eingehen, werden durch Gruppenprozesse beeinflusst. Für Gehlbach (2004a) gab das Modell An- 
regung zur Konstruktion eines Fragebogens zur Erfassung von historical empathy bei Schülern der neunten und zehnten Klassenstufe. Ausgehend von zwei Comics zum Wahlsystem im Antiken Griechenland und in den USA um das Jahr 1900 formulierte er Items, die sich an die in Tabelle 1 beschriebenen Levels anlehnen und die Fähigkeit von Schülern zur historischen Perspektivenübernahme erfassen sollen. Über die Güte des Instruments und die empirische Bestätigung der von Ashby und Lee (1987) aufgestellten Abfolge macht er jedoch keine Angaben, er nutzt das Instrument vielmehr als einen Indikator um Beziehungen zwischen sozialer und historischer Perspektivenübernahme empirisch zu untersuchen.

\subsection{Bewertung der Kompetenzmodelle im Hinblick auf ihre Anschlussfähigkeit für die empirische Erforschung historischer Perspektivenübernahme}

Alle in diesem Kapitel dargestellten fachdidaktischen Kompetenzmodelle weisen der Kompetenz, historische Perspektiven einzunehmen, einen hohen Stellenwert zu. Sowohl in den Modellen von Sauer (2006), Gautschi (2006) und der FUERKooperation (Körber, Schreiber et al., 2007) als auch in den nationalen und internationalen Bildungsstandards wird historische Perspektivenübernahme als wichtiger Bestandteil historischen Denkens angesehen, der zudem Brücken zwischen Vergangenheit und Gegenwart bauen kann und soll.

Mit diesen eher allgemeinen Vermutungen und Forderungen enden jedoch schon die Gemeinsamkeiten zwischen den Modellen in Bezug auf das Verständnis von historischer Perspektivenübernahme. Mit ähnlichen Begrifflichkeiten umschreiben die Autoren durchaus unterschiedliche Dinge. Perspektivenübernahme schließt bei Gautschi (2006) beispielsweise Mitgefühl mit historischen Personen ein - davon ist bei Sauer (2006) oder der FUER Gruppe (2007) nicht die Rede. Auch in der angloamerikanischen Literatur wird diese Verknüpfung abgelehnt (Foster, 2001; Lee \& Ashby, 2001). Weiterhin ist in allen Kompetenzmodellen der Begriff Fremdverstehen präsent, jedoch lässt sich nur spekulieren, was die einzelnen Autoren im Detail darunter verstehen - ein gemeinsamer theoretischer Bezugsrahmen existiert nicht.

Ein direkter Vergleich der Modelle im Hinblick auf die Kompetenz zur historischen Perspektivenübernahme erscheint problematisch, da sich die Ansätze in Bezug auf ihre Zuschnitte und Adressaten stark unterscheiden. Das Modell von Körber, 
Schreiber et al. (2007) stellt eine in sich geschlossene geschichtstheoretisch orientierte Konzeption dar, in die sich Perspektivenübernahme eingliedern lässt. Es bietet vielerlei Anschlussmöglichkeiten für den geschichtswissenschaftlichen Diskurs über historisches Denken, lässt sich jedoch aufgrund seines hohen Abstraktionsgrades nur schwer auf den Schulalltag übertragen. Ein Lehrer, der nach Hinweisen sucht, wie er die Kompetenz zur historischen Perspektivenübernahme bei seinen Schülern fördern kann, wird sich vermutlich eher an den checklistenartigen Kompetenzmodellen von Sauer (2006) oder Gautschi (2006) orientieren. Dort wird er hauptsächlich Schlagworte finden, die mit dieser Kompetenz zusammenhängen und die intuitiv plausibel erscheinen. Er bekommt jedoch keine theoretisch konsistenten Erklärungen, welche zentralen historischen Einsichten bei Schülern unterschiedlicher Kompetenzniveaus historische Perspektivenübernahme konstituieren.

Bezogen auf die Anschlussfähigkeit für eine empirische Erfassung historischer Perspektivenübernahme können die vorgestellten Modelle bisher nur ansatzweise genutzt werden. Dies ist auf einen generellen Mangel an Rückbindung an die bereits existierenden theoretischen und empirischen Befunde zu dieser Kompetenz zurückzuführen. In keinem der Modelle wird explizit auf theoretische Konzeptionen zur historischen Perspektivenübernahme Bezug genommen (z.B. Bergmann, 2000; Davis Jr., Yeager \& Foster, 2001). Auch die Rezeption psychologischer Theorien zur Perspektivenübernahme und -koordination (vgl. Kapitel 2) findet nicht statt. Dies lässt sich analog für die mangelnde Integration empirischer Befunde kritisieren. Die Arbeiten von Ashby und Lee (1987) oder von Borries (1994; 1995), aus denen sich Hinweise für die Theoriebildung, die Operationalisierung und mögliche Kompetenzunterschiede ableiten lassen, finden in den Kompetenzmodellen und Bildungsstandards keinerlei explizite Berücksichtigung. Dieses Theorie- und Empiriedefizit schränkt die Aussagekraft und die Anschlussfähigkeit der Kompetenzmodelle für die Erforschung von Einzelkompetenzen stark ein. Es ist zwar plausibel, dass historische Perspektivenübernahme wichtig für historisches Lernen und darüber hinaus auch relevant für das gegenwärtige Handeln von Schülern ist; ohne eine genaue Definition der verwendeten Begriffe unter Rückbezug auf Theorien und empirische Befunde bleibt der Nutzen für die fachdidaktische Kompetenzforschung jedoch geringer als prinzipiell möglich. 


\section{Perspektivenübernahme als psychologisches Konstrukt mit An- schlussmöglichkeiten für die Erforschung historischen Verstehens}

\subsection{Vorbemerkungen}

Im zweiten Kapitel werden theoretische Ansätze und empirische Befunde zum Konstrukt der sozialen Perspektivenübernahme dargestellt, die Anschlussmöglichkeiten für die Erforschung historischen Verstehens bieten. Insbesondere Selmans Modell der sozialen Perspektivenkoordination eignet sich dafür, vornehmlich aus zwei Gründen. Erstens stellt es als Entwicklungsmodell einen geeigneten Rahmen für die Betrachtung von Schülerkompetenzen dar, da wir es mit Personen zu tun haben, deren Perspektivenübernahme nicht vollständig ausdifferenziert, sondern noch in Veränderung begriffen ist. Zweitens liefert das Modell Annahmen zu qualitativen Entwicklungsfortschritten im Denken von Schülern, die im Hinblick auf die Formulierung von Kompetenzstufen fruchtbarer erscheinen als rein quantitative Differenzen. Ergänzt wird Selmans entwicklungspsychologischer Ansatz um theoretische Ansätze sowie empirische Befunde zur Kontextspezifität von Perspektivenübernahme. Erst die Berücksichtigung solcher Faktoren, die außerhalb der universellen sozial-kognitiven Struktur von Individuen liegen, ermöglicht die interdisziplinäre Verknüpfung zwischen der Fähigkeit zur sozialen Perspektivenübernahme und den fachdidaktischen Annahmen zu Perspektivenübernahme als historischer Kompetenz.

\subsection{Das Modell der sozialen Perspektivenkoordination nach Robert L. Selman}

Die Fähigkeit von Kindern und Erwachsenen, in sozialen Interaktionen die Perspektiven anderer Personen einzunehmen und diese mit dem eigenen Standpunkt in Beziehung zu setzen, beschäftigt die Arbeitsgruppe um Robert L. Selman etwa seit den siebziger Jahren. Das Modell zur sozialen Perspektivenkoordination (Selman, 1980) lässt sich als sozial-kognitive strukturgenetische Entwicklungstheorie charakterisieren und knüpft damit an die Arbeiten von Piaget, Mead und Kohlberg zur (sozial-) kognitiven und moralischen Entwicklung an. Selman (1980) versteht Perspektivenkoordination als sozial-kognitive Fähigkeit, die sich über die ersten zwei bis drei 
Lebensjahrzehnte entwickelt und die durch zunehmende Strukturierung, Ausdifferenzierung und Integration gekennzeichnet ist. Die Entwicklungsstufen, die er postuliert, sind als qualitativ voneinander abgrenzbare Einheiten anzusehen. Jede höhere Stufe erfordert eine Restrukturierung von Fähigkeiten der unteren Stufen und einen qualitativen Zugewinn an sozial-kognitiven Fähigkeiten. Selman (1980) postuliert fünf Entwicklungsstufen, die folgendermaßen definiert sind:

Auf Stufe 0 (undifferentiated, egocentric; ca. 3 bis 6 Jahre) können physische und psychische Charakteristika von Personen noch nicht klar voneinander getrennt werden. Es gibt noch keine Unterscheidung zwischen Handlungen und Gefühlen, zwischen intentionalem und nicht-intentionalem Verhalten. Die Wahrnehmung von unterschiedlichen Perspektiven ist auf dieser Stufe noch nicht möglich.

Auf Stufe 1 (differentiated, subjective; ca. 5 bis 9 Jahre) können Personen physische und psychische Charakteristika voneinander getrennt wahrnehmen. Jeder Person wird auf dieser Stufe eine subjektive und einzigartige Wahrnehmung zugeschrieben. Das in Beziehung Setzen von mehreren Perspektiven erfolgt jedoch noch einseitig, indem nur ein Handelnder berücksichtigt wird, auf den verschiedene soziale Interaktionen wirken. Gefühle von anderen Personen werden auf dieser Stufe als direkt beobachtbar angesehen.

Der wichtigste Fortschritt auf Stufe 2 (reciprocal, self-reflective; ca. 7 bis 12 Jahre) besteht darin, dass es möglich ist, gedanklich die eigene Perspektive zu verlassen und Gedanken und Handlungen einer anderen Person nachzuvollziehen. Dies geschieht im Bewusstsein, dass auch anderen Personen dieser Schritt möglich ist. Es wird verstanden, dass Menschen sich inkongruent zu ihren Gefühlen verhalten können. Sowohl die eigene als auch die Perspektiven von anderen werden anerkannt, können jedoch noch nicht simultan koordiniert werden.

Auf Stufe 3 (mutual, third-person; ca. 10 bis 15 Jahre) wird es möglich, nicht nur die eigene aktuelle Perspektive zu verlassen, sondern das Selbst als Ganzes zu verlassen und somit eine echte Dritte-Person-Perspektive einzunehmen. Personen auf dieser Stufe können sich selbst sowohl als Handelnde als auch als Objekt innerhalb einer Interaktion betrachten. Die eigene Perspektive und die der anderen können simultan berücksichtigt und koordiniert werden und die Interaktion kann von einer generalisierten Perspektive aus betrachtet werden.

Stufe 4 (intimate, in-depth, societal; ca. 12 Jahre bis ins Erwachsenenalter) schließt die Entwicklung der Perspektivenkoordination ab. Kennzeichnend für diese 
Stufe ist die Erkenntnis, dass nicht alle Motive und Emotionen von Personen selbstreflexiv erschlossen werden können. Es wird auf dieser Stufe erkannt, dass es Gefühle gibt, die Personen haben, ohne selbst zu verstehen, warum sie sie haben. Weiterhin wird auf dieser Stufe erst erschlossen, dass Beziehungen und Kommunikation auf verschiedenen Ebenen stattfinden können (von der Ebene oberflächlicher Informationen bis zur Ebene tieferer Gefühle).

In dem Modell wird Perspektivenkoordination als eine Fähigkeit verstanden, die das Handeln von Personen in ihren sozialen Gefügen bestimmt. Aus diesem Grund zieht Selman den Begriff der sozialen Perspektivenkoordination gegenüber dem der sozialen Perspektivenübernahme vor. Er stellt heraus, dass Perspektivenkoordination stärker die Handlungsrelevanz betont, die für die Bewältigung sozialer Situationen erforderlich ist (Selman, 2003). Auch die Inhaltsbereiche, an denen er sein Modell entwickelt hat, weisen diesen deutlichen Alltags- und Handlungsbezug auf. Mithilfe sozialer Dilemmasituationen und halbstrukturierten Interviews weist er die strukturellen Veränderungen bei Kindern und Jugendlichen an vier Inhaltsbereichen (domains) nach. Diese Domains sind: Denken über Personen, Denken über Freundschaften, Denken über Gleichaltrigengruppen und Denken über Eltern-Kind-Beziehungen.

Die Validität der von Selman postulierten Stufen wurde in diversen empirischen Studien nachgewiesen. Gurucharri und Selman (1982) bestätigten die Stufenabfolge in einer Längsschnittstudie über einen Zeitraum von fünf Jahren. Die Bedeutung von Perspektivenkoordination für das Sozialverhalten von Kindern und Jugendlichen konnte mehrfach nachgewiesen werden (z.B. Adalbjarnardottir, 1995; Beardslee, Hickey Schultz \& Selman, 1987; Yeates \& Selman, 1989). Ein dritter Aspekt der Validität eines solchen strukturgenetischen Stufenmodells betrifft die Unempfindlichkeit gegenüber Kontextfaktoren. Theorien, die eine universelle Entwicklungsabfolge postulieren, sollten unabhängig vom untersuchten Inhaltsbereich Gültigkeit haben. Auch diesem Anspruch scheint das Modell gerecht zu werden, zumindest liefert Selman (1980) überzeugende (wenn auch vornehmlich querschnittlich erhobene) empirische Anhaltspunkte dafür, dass Perspektivenkoordination über verschiedene Inhaltsbereiche (Denken über Individuen, Freundschaften, Peer-Gruppen) hinweg dieselbe Struktur aufweist. Gleichzeitig weist er jedoch bereits 1980 darauf hin, dass Variationen in Entwicklungsverläufen, die auf Kontextfaktoren zurückzuführen sind, für die entwicklungspsychologische Forschung von großem Interesse sein könnten. Dies ist insbesondere bemer- 
kenswert, da in der Entwicklungspsychologie zu jener Zeit der Anspruch an die strukturelle Ganzheit in der Konzeption von Entwicklungsmodellen ${ }^{6}$ vorherrschend war.

\subsection{Perspektivenübernahme als kontextabhängige Kompetenz}

\subsubsection{Kontextabhängigkeit von Perspektivenübernahme und sozio-moralischem Urteil aus entwicklungspsychologischer Sicht}

Die Annahme, dass sich Perspektivenübernahme nach universellen Prinzipien und weitgehend unabhängig von spezifischen sozialen Kontexten entwickelt, wurde und wird bis heute facettenreich und kontrovers diskutiert. In der Entwicklungspsychologie beschränkt sich diese Diskussion nicht auf die Fähigkeit zur sozialen Perspektivenübernahme und -koordination, sondern schließt ebenfalls das Forschungsfeld der Moralentwicklung ein, für die Perspektivenübernahme als eine zentrale Voraussetzung angesehen werden kann (Keller \& Edelstein, 1991). Ein Verfechter der Kontextspezifität von Perspektivenübernahme und moralischem Urteil ist Elliot Turiel. Seiner Ansicht nach unterscheiden sich verschiedene Inhaltsbereiche, in denen sozio-moralisches Urteil gefragt ist, so grundlegend voneinander, dass es nicht möglich ist, das Denken von Personen in diesen Inhaltsbereichen im Sinne einer strukturellen Ganzheit zu beschreiben, die sich in qualitativen Stufen entwickelt. Sozio-moralisches Denken ist demnach immer das Ergebnis einer Interaktion zwischen dem Individuum und der spezifischen Situation. Dabei ist die Koordination verschiedener sozialer Perspektiven vor allem als eine Methode zur Aneignung von sozialem Wissen anzusehen, die im Laufe der Entwicklung allenfalls quantitativen Veränderungen (z.B. größere Genauigkeit oder breitere Anwendbarkeit) unterliegt (z.B. Turiel, 1983). Eine endgültige Entscheidung für den einen oder anderen Pol (vollständige Universalität vs. vollständige Kontextbedingtheit) scheint zum jetzigen Zeitpunkt nicht möglich zu sein. Für beide Ansichten gibt es zahl-

\footnotetext{
${ }^{6}$ Die Forderung nach struktureller Ganzheit (structured wholeness) steht ebenfalls in der Tradition der Arbeiten von Piaget (z.B. 1970) und Kohlberg (z.B. Colby, Kohlberg, Gibbs \& Lieberman, 1983). Danach bilden die am Urteilsdenken beteiligten internen Instanzen eine kognitive Struktur, eine strukturelle Ganzheit, die je nach Entwicklungsstand eine bestimmte stufenspezifische Qualität aufweist. Daraus resultiert die Überlegung, dass aus ein und derselben Struktur nur dieselbe Argumentationsqualität hervorgehen kann, unabhängig vom Inhaltsbereich, in dem eine Argumentation gefragt ist.
} 
reiche Belege, die beispielsweise von Smetana (2006) zusammengefasst werden. Da die soziale Welt jedoch durch Heterogenität sowie die Koexistenz unterschiedlichster sozialer Orientierungen, Motivationen und Zielvorstellungen charakterisiert ist, sind Kontextfaktoren bei der Erforschung sozio-kognitiven und sozio-moralischen Denkens zu berücksichtigen (Smetana, 2006). Diese betreffen beispielsweise die soziale Schicht der Befragten und insbesondere den kulturellen und ethnischen Kontext, in dem die Befragten aufgewachsen sind (Keller, Edelstein, Krettenauer, Fu-xi \& Ge, 2005; Keller, Lourenço, Malti \& Saalbach, 2003; Quintana, Ybarra, Gonzalez-Doupe \& De Baess, 2000; Smetana, Killen \& Turiel, 1991). Neben solchen Belegen für den Einfluss vornehmlich demographischer Merkmale liefert die Studie von Wainryb (1993) Erkenntnisse darüber, wie stark Personen ihre eigenen sozio-moralischen Maßstäbe generalisieren und auf andere kulturelle Kontexte übertragen. Mithilfe eines experimentellen Designs zeigt die Autorin, dass universell gültige vs. relative und kontextbedingte sozio-moralische Urteile nicht aufgrund von Entwicklungsunterschieden zustande kommen, sondern von den spezifischen Informationen über die fremde Kultur abhängen, die dem Individuum vorliegen. Werden Informationen zur Verfügung gestellt, die darauf hinweisen, dass in einer fremden Kultur ,,andere Realitäten“ vorherrschen (z.B. andere Vorstellungen darüber, was das menschliche Leben ausmacht, über das Zustandekommen von Krankheiten oder gar den Einfluss von Geistern auf das menschliche Leben), werden eigene moralische Urteile relativiert und an den spezifischen kulturellen Kontext angepasst.

Feigenberg, King, Barr und Selman (2008) untersuchten, wie sich die Fähigkeit zur Perspektivenkoordination im Kontext Schule auf Handlungsstrategien von Schülern auswirken kann. Anhand eines Fallbeispiels über die soziale Ausgrenzung einer Schülerin wurden Schüler basierend auf Selmans theoretischen Annahmen (2003) dazu befragt, wie sie handeln würden, wenn sie eine solche Situation entweder als Außenstehender (bystander) erleben würden oder zur aktiven Teilnahme an der Ausgrenzung aufgefordert würden. Zusätzlich sollten sie die von ihnen genannten Handlungsstrategien begründen und rechtfertigen. Die Studie führte zu dem Ergebnis, dass sowohl die Auswahl von Handlungsstrategien als auch deren Rechtfertigung massiv von Faktoren im unmittelbaren Umfeld der Schüler beeinflusst wird. Beispielsweise wählten Schüler, die ihre Schule als Raum wahrnahmen, in dem Mitbestimmung erwünscht ist, vermehrt Handlungsstrategien, die dem Opfer der Ausgrenzung halfen, und auch ihre Rechtfertigungen für diese Handlungen waren weniger konventionell und regelorientiert, sondern 
enthielten öfter transformationale Elemente (z.B. Möglichkeit einer positiven Entwicklung aufseiten der Täter durch eigenes Verhalten, Veränderung der Gruppendynamik).

\subsubsection{Perspektivenübernahme als multidimensionale soziale Kompetenz.}

Die bisher behandelten theoretischen Ansätze und empirischen Befunde stellen Perspektivenübernahme und -koordination als eine Kompetenz dar, die sich im Laufe des Kindes- und Jugendalters entwickelt und mehr oder weniger durch Kontextfaktoren beeinflusst werden kann. Gehlbach (2004a; 2004b) schlägt eine alternative Konzeption von sozialer Perspektivenübernahme als multidimensionale Kompetenz vor, die sowohl personenspezifische als auch situationsspezifische Charakteristika einschließt. ${ }^{7}$ Die situationsspezifischen Charakteristika unterteilt Gehlbach (2004b) in (a) Merkmale des weiter gefassten Kontextes (z.B. die Kooperativität innerhalb der Schule oder Schulklasse oder von der Aufgabe ablenkende Umgebungsfaktoren) sowie (b) Merkmale der konkreten Aufgabe, in der Perspektivenübernahme verlangt wird.

$\mathrm{Zu}$ den Merkmalen der konkreten Perspektivenübernahme-Aufgabe gehört der Bekanntheitsgrad der Person, deren Perspektive eingenommen werden soll. Gehlbach (2004b) fasst sozialpsychologische Befunde zusammen, die zeigen, dass es leichter ist, die Perspektive von Freunden und Familienmitgliedern einzunehmen als die von fremden Personen. Ein weiteres Merkmal der Perspektivenübernahme-Aufgabe ist die Temporalität. Empirische Befunde, inwieweit sich die Übernahme gegenwärtiger, vergangener und zukünftiger Perspektiven voneinander unterscheiden, existieren bisher nicht. Gehlbach vermutet jedoch, dass Perspektivenübernahme am besten in gegenwärtigen Situationen funktioniert, da über die Gegenwart meist mehr und akkuratere Informationen vorliegen als über Vergangenheit und Zukunft. Ein drittes Merkmal betrifft den Realitätsgehalt der Perspektivenübernahme-Aufgabe. Auch für diesen Aspekt liegen bisher keine empirischen Studien vor, in denen reale mit hypothetischen Perspektivenübernahme-Aufgaben direkt verglichen werden. Gehlbach vermutet, ähnlich wie für die Temporalität, dass realitätsnahe Situationen zu besserer Perspektivenübernahmeleistung beitragen. Als weiteres Merkmal führt Gehlbach die Entschlüsselbarkeit der Person an,

\footnotetext{
${ }^{7}$ Gehlbach (2004b) verwendet in Anlehnung an die Arbeiten von Snow (1996) den Begriff aptitude. Dieser impliziert, dass Ausprägungen von Perspektivenübernahme nicht nur aufgrund der bei Individuen verfügbaren Bereitschaften und Fähigkeiten zustande kommen, sondern ebenfalls von der Interaktion zwischen Person und Situation abhängen.
} 
deren Perspektive übernommen werden soll. Perspektiven von Personen, die wenig Emotionen zeigen und auch sonst wenig Informationen über ihre Handlungsabsichten und Beweggründe liefern, können vermutlich schlechter eingenommen werden als die von Personen, die ihre Gedanken und Gefühle deutlicher artikulieren. Weitere Merkmale betreffen die Dauer und Erfahrung mit Perspektivenübernahme-Aufgaben. Wie in vielen Bereichen ist es auch in Bezug auf Perspektivenübernahme wahrscheinlich, dass zunehmende Übung und Erfahrung mit sozialen Interaktionen die Perspektivenübernahmeleistung verbessern. Gehlbach (2004b) vermutet, dass größere Erfahrung auch eine mögliche Erklärung für die entwicklungspsychologischen Befunde von Selman (1980) sowie Eisenberg, Murphy und Shepard (1997) darstellen könnte. Ein letztes Merkmal der konkreten Perspektivenübernahme-Aufgabe betrifft den Einfluss moralisch konnotierter Situationen auf die Perspektivenübernahme-Leistung. Gehlbach diskutiert diesen Aspekt nur am Rande, vermutet aber, dass die kognitive Komplexität durch eine moralische Dimension erhöht wird und Perspektivenübernahme in solchen Situationen deshalb schwieriger zu bewältigen ist.

\subsection{Soziale Perspektivenübernahme im historischen Kontext - Versuch einer Synthese}

Perspektivenübernahme scheint eine Fähigkeit zu sein, die sich nach Selman (1980) weitgehend universell in Form von qualitativ unterscheidbaren Stufen entwickelt, die jedoch zugleich von diversen Kontextfaktoren beeinflusst werden kann. Dieses in der psychologischen Diskussion bestehende Spannungsfeld lässt die Frage aufkommen, wie sich die soziale Kompetenz zur Perspektivenübernahme im Kontext Geschichte darstellt.

Aufgrund der bisher dargestellten Ansätze aus Geschichtsdidaktik und Psychologie lassen sich diverse theoretische und empirische Bezüge zwischen sozialer und historischer Perspektivenübernahme herstellen, die zum Abschluss dieses Kapitels näher erläutert werden sollen. So sind beispielsweise Überschneidungen zwischen den fachdidaktischen Kompetenzmodellen und dem Modell zur sozialen Perspektivenkoordination von Selman (1980) feststellbar. Weiterhin liefern Gehlbachs Arbeiten (2004a; 2004b) Anknüpfungspunkte für die Betrachtung verschiedener Dimensionen historischer Perspektivenübernahme. Und schließlich ergänzen sich die beiden Disziplinen, 
wenn es um die angestrebten Bildungsziele geht, die mit dem Einnehmen historischer Perspektiven verbunden sind.

\subsubsection{Fachdidaktische Kompetenzannahmen und das Modell zur sozialen Perspektiven- koordination}

Die Kompetenzmodelle von Sauer (2006) und der FUER-Gruppe (Körber, Schreiber et al., 2007) sowie das auf den Gegenstand historical empathy zugeschnittene Modell von Ashby und Lee (1987) lassen sich mit Selmans Annahmen zur sozialen Perspektivenkoordination $(1980 ; 2003)$ in Verbindung bringen, da alle drei mögliche Entwicklungsfortschritte thematisieren.

Selman (1980) sieht den Egozentrismus als Ausgangspunkt für die Entwicklung von Perspektivenübernahme an und weist empirisch nach, dass diese Denkweise bei Kindern im Alter von drei bis sechs Jahren vorherrschend ist. Die didaktischen Modelle machen demgegenüber keine Altersangaben, es ist jedoch zu vermuten, dass die Autoren ältere Kinder im Blick hatten, da Geschichte im Regelfall erst ab der Sekundarstufe 1 als eigenständiges Fach unterrichtet wird. Dennoch sind Annahmen zum Egozentrismus auch dort relevant: Ashby \& Lee (1987) verorten auf Level 1 Kinder, die alles Vergangene als unverständlich und rückständig wahrnehmen, weil es nicht mit ihren eigenen Vorstellungen von der Welt vereinbar ist. In Sauers (2006) Teilkompetenz Fremdverstehen soll diese Denkweise bereits als Ziel der ersten Unterkompetenz überwunden werden. Egozentrismus findet sich bei Ashby und Lee (1987) auch noch auf Level 3, wenn auch in einer etwas moderateren Form: Kinder übertragen heutige Vorstellungen direkt auf vergangene Situationen und entwickeln auf deren Basis gegenwartsfixierte Handlungsvorstellungen für die historischen Akteure. Mit dieser Denkweise scheint zwar die ausschließlich negative Bewertung der historischen Akteure und Institutionen von Level 1 überwunden; zu einer historisch plausiblen Erklärung, die Unterschiede und Gemeinsamkeiten zwischen Gegenwart und Vergangenheit berücksichtigt, sind die Schüler jedoch noch nicht in der Lage.

Die Stufen zwei und drei aus Selmans (1980) Modell werden in den geschichtsdidaktischen Überlegungen auf verschiedene Weise aufgegriffen. Sauer (2006) formuliert für die Teilkompetenz Mit Perspektivität in der Geschichte umgehen, dass Schüler sich in unterschiedliche Perspektiven hineinversetzen können und in ihnen sprechen und 
handeln. Diese Kompetenz könnte man auf Selmans Stufe 2 verorten, wenn man annimmt, dass damit noch nicht die simultane Koordination von Perspektiven gemeint ist. Diese wäre dann sowohl Bestandteil von Selmans Stufe 3 als auch von Sauers dritter Unterkompetenz (historische Situationen und Ereignisse parallel aus unterschiedlichen Perspektiven betrachten). Bezogen auf das Modell der FUER-Gruppe (Körber, Schreiber et al., 2007) scheinen Selmans Stufen zwei und drei am ehesten dem intermediären Niveau zu entsprechen, in dem konventionelle Operationen wirksam sind. Solche Ansätze konventionellen Denkens lassen sich auch im Modell von Ashby und Lee (1987) auf Level 2 feststellen. Dort werden stereotype Vorstellungen über Rollen und Funktionen historischer Akteure aktiviert, die deren Handlungen erklären sollen. Spezifische Charakteristika des historischen Kontexts können Kinder auf diesem Level jedoch noch nicht einbeziehen.

Die höchste Stufe sozialer Perspektivenkoordination besteht bei Selman (1980) darin, dass Menschen in der Lage sind, Beziehungen und Interaktionen in ihrer Bedeutung und Intensität voneinander zu unterscheiden und ihre Handlungen darauf abstimmen. Außerdem erlangen Menschen tiefere Einsichten über die Selbstreflexionsfähigkeit der eigenen und anderer Personen. Die Bezeichnung dieser Stufe 4 als in-depth, societal lässt zudem Schlüsse in Bezug auf die gesellschaftliche und zeitliche Verankerung sozialer Interaktionen zu. Damit werden Selmans Annahmen anschlussfähig an die didaktischen Modelle zur historischen Perspektivenübernahme, z.B. an Level 5 bei Ashby und Lee (1987). Schüler, die auf diesem Level argumentieren, können die weiteren historischen Kontextbedingungen einbeziehen, wenn sie über spezifische Situationen und das Verhalten historischer Akteure nachdenken. Der Fokus wird somit von der Einzelsituation erweitert, bei Selman auf den gegenwärtigen, bei Ashby und Lee auf den historischen Kontext. Im Modell der FUER-Gruppe (Körber, Schreiber et al., 2007) ist mit einem hohen Niveau von Welt- und Fremdverstehen impliziert, dass Menschen zur Reflexion eigener Handlungen fähig sind und Interaktionen in ihrer gesellschaftlichen Abhängigkeit verstehen können. Dafür scheint Perspektivenkoordination, wie Selman sie konzipiert, eine unverzichtbare Voraussetzung zu sein. Wie sollte es Menschen sonst möglich sein, das Verhältnis historischer Ereignisse und Prozesse zu ihrer eigenen Welt und Zeit zu überdenken sowie kulturelle und intersubjektive Differenzen zu reflektieren, wie es Schreiber (2007) fordert? 


\subsubsection{Historische Perspektivenübernahme als multidimensionales Konstrukt?}

Gehlbachs (2004a; 2004b) Arbeiten über Perspektivenübernahme als multidimensionales Konstrukt lassen den Kontext Geschichte als einen besonders anspruchsvollen erscheinen: Der Gegenstand historischer Perspektivenübernahme ist per definitionem das Vergangene (Merkmal: Temporalität). Gedanken und Handlungen der historischen Akteure sind uns nur vermittelt über Quellen und Darstellungen zugänglich und wir können keine Beziehungen zu ihnen aufbauen, die für uns von direkter persönlicher Relevanz wären (Merkmale: Entschlüsselbarkeit und Bekanntheitsgrad). All diese Herausforderungen müssten nach Gehlbach (2004b) dazu führen, dass sowohl im Hinblick auf die Fähigkeit als auch auf die Bereitschaft, sich in historische Akteure hineinzuversetzen, mit Einbußen gerechnet werden muss. Gleichzeitig stellen diese Merkmale jedoch auch Anhaltspunkte für eine Förderung von historischer Perspektivenübernahme im Geschichtsunterricht dar: Während die zeitliche Komponente unveränderbar ist, könnte die persönliche Relevanz historischer Ereignisse für die Gegenwart der Schüler stärker vom Lehrer betont werden um historische Perspektivenübernahme zu fördern. Auch die Entschlüsselbarkeit historischer Akteure kann durch eine geeignete Auswahl von Quellen und Darstellungen vom Lehrer erhöht werden. Wie wichtig die Verfügbarkeit von Informationen für das Einnehmen fremder Perspektiven ist, lässt sich auch aus der Studie von Wainryb (1993) schlussfolgern. Je mehr Informationen über die speziellen kulturellen und historischen Kontextbedingungen Schüler erhalten, desto leichter sollte es für sie sein, ihre eigenen Wertvorstellungen zu relativieren und nicht ungeprüft auf historische Kontexte zu übertragen - ein erklärtes Ziel des Geschichtsunterrichts im Allgemeinen und der historischen Perspektivenübernahme im Speziellen (z.B. Bergmann, 2000).

\subsubsection{Ziele historischer und sozialer Perspektivenübernahme}

Bezogen auf die unmittelbaren Ziele von Perspektivenübernahme bestehen unterschiedliche Auffassungen in Psychologie und Fachdidaktik. Als soziale Kompetenz dient Perspektivenübernahme der Verbesserung sozialer Interaktionen und der Lösung von Konflikten (z.B. Gehlbach, 2004a; Gutzwiller-Helfenfinger, 2003; Selman, 2003). In der didaktischen Diskussion hat Perspektivenübernahme eine primär analytische 
Funktion. Da zwischen Historiker bzw. Schüler und den historischen Akteuren in der Regel keine direkten Interaktionen bestehen, kann es nur um die nachträgliche historisch plausible Rekonstruktion vergangener Ereignisse gehen. Ziel ist das Verstehen historischer Akteure und die Analyse historischer Denk- und Handlungshorizonte (z.B. Lee \& Ashby, 2001; Sauer, 2006; Yeager \& Foster, 2001).

Nimmt man jedoch die übergeordneten Bildungsziele in den Blick, die mit der Förderung historischer Perspektivenübernahme im Schulkontext verfolgt werden, wird die Verknüpfung zwischen fachspezifischer und sozial-kognitiver Kompetenz offensichtlich. Denn die Begründung, warum das Fach Geschichte und speziell das Verständnis historischer Perspektiven für Schüler unerlässlich ist, ist in der Mehrzahl der Kompetenzmodelle und Bildungsstandards doch sehr gegenwartsbezogen: Kritikfähigkeit, Toleranz und die Anerkennung fremder moralischer Maßstäbe sind nur drei der vielen Schlagworte, mit denen der erhoffte Nutzen von historischer Perspektivenübernahme unterstrichen wird. Ashby und Lee (1987) formulieren ihre hohen Erwartungen folgendermaßen: „Pupils who [...] have learnt the kind of moves which help them to achieve empathy, have not just taken a step forward in their history, but are more likely to be able to cope with the present world" (S. 64). Bisher fehlen jedoch empirische Belege dafür, ob historische Perspektivenübernahme als eine fachspezifische Kompetenz tatsächlich mit sozial-kognitiven Fähigkeiten und weiteren Indikatoren sozialer Kompetenz zusammenhängt und in welcher Wirkrichtung diese beiden Konzepte bei Schülern zueinander stehen. 


\section{Forschungsziele}

Die psychologische Untersuchung historischer Kompetenzen bedeutet in vielerlei Hinsicht Neues auszuprobieren ohne sich auf viel Althergebrachtes stützen zu können. Das gilt auch für den Gegenstand dieser Arbeit - die Kompetenz zur historischen Perspektivenübernahme. Weder liegt ein in sich kohärenter theoretischer Rahmen vor, aus dem spezifische Hypothesen über Schülerkompetenzen abgeleitet werden können, noch existieren geeignete Messzugänge um historische Kompetenzen im Hinblick auf die Einführung von Bildungsstandards erfassen zu können.

Mit den ersten beiden Kapiteln wurden die theoretischen Grundlagen aufgezeigt, an denen sich die empirische Erfassung historischer Perspektivenübernahme orientieren soll. Dies sind zum einen die vornehmlich normativen Setzungen aus den Kompetenzmodellen und Bildungsstandards der Geschichtsdidaktik, die durch erste empirische Befunde wie die von Ashby und Lee (1987) flankiert werden. Zum anderen sind psychologische Theorien heranzuziehen, insbesondere das Modell zur sozialen Perspektivenkoordination von Selman (1980), welches weitgehend universelle Entwicklungsschritte dieser Kompetenz aufzeigt. Ergänzt durch empirische Befunde und alternative theoretische Konzeptionen (z. B. Gehlbach, 2004b, Turiel, 1983) entsteht ein Bild einer sozial-kognitiven Kompetenz, das diverse Kontextfaktoren einschließt und dadurch anschlussfähig für die Betrachtung von Perspektivenübernahme im Kontext Geschichte wird. Basierend auf dieser Auseinandersetzung mit fachdidaktischen und psychologischen Grundlagen wird als Ausgangspunkt für die empirischen Untersuchungen folgende Arbeitsdefinition von Perspektivenübernahme als Kompetenz historischen Verstehens vorgeschlagen:

Historische Perspektivenübernahme ist eine sozial-kognitive Kompetenz, sich in Akteure aus der Vergangenheit hineinzuversetzen und dabei über die eigene gegenwärtige Sichtweise hinaus historische Denk- und Handlungshorizonte auf Basis der damals geltenden Bedingungen zu rekonstruieren.

Ziel der vorliegenden Arbeit ist ausgehend von dieser Arbeitsdefinition historischer Perspektivenübernahme zunächst die Entwicklung eines Messinstrumentes. Dies soll vor allem drei Anforderungen erfüllen: 
a)

Sowohl fachdidaktische Überlegungen als auch psychologische Theorien sollen herangezogen werden um zu bestimmen, wie sich kompetente von weniger kompetenten Schülerantworten unterscheiden lassen.

b) Das Instrument soll eine standardisierte Erfassung größerer Schülerstichproben ermöglichen um im Hinblick auf die Umsetzung von Bildungsstandards für das Fach Geschichte erste Vorarbeiten zu leisten.

c) Historische Perspektivenübernahme soll bei Schülern mit einem Instrument erfasst werden, welches den psychometrischen Anforderungen an Reliabilität und Validität genügt.

Das in dieser Arbeit entwickelte Messinstrument wird in Kapitel 4 anhand einer Publikation (erschienen in der Zeitschrift Learning and Individual Differences) dargestellt. Die dort berichteten Ergebnisse basieren auf den Daten einer Stichprobe von Gymnasiasten der 10. Klasse. Ergänzt werden diese Analysen um einige Befunde aus einer Stichprobe von Siebtklässlern. Es handelt sich dabei um Ausschnitte aus einem Kapitel des Buches Forschungswerkstatt Geschichtsdidaktik empirisch 07.

Anschließend an die Vorstellung des Messinstrumentes wird in Kapitel 5 der Frage nachgegangen, wie die Kompetenz zur historischen Perspektivenübernahme sowohl mit fachspezifischen Schülermerkmalen (beispielsweise der Geschichtsnote und dem fachspezifischen Interesse) als auch mit der Fähigkeit zur sozialen Perspektivenübernahme zusammenhängt. Diese Frage, die sich aus dem interdisziplinären Hintergrund der Arbeit ableiten lässt und insbesondere an die theoretischen und empirischen Argumente zur Kontextspezifität von Perspektivenübernahme anknüpft (vgl. Abschnitte 2.3 und 2.4), wird basierend auf einer weiteren Publikation (im Druck bei der Zeitschrift für Erziehungswissenschaft) diskutiert. Ausgehend von der vergleichenden Analyse der Daten aus den Klassenstufen 7 und 10 werden erste empirisch gestützte Vermutungen über die Wirkzusammenhänge zwischen fachspezifischen und sozial-kognitiven Schülermerkmalen bezogen auf den Kontext Geschichte aufgestellt. 


\section{Analysen zur Qualität eines neuen Messinstrumentes zur Erfassung historischer Perspektivenübernahme}

\subsection{Historical Perspective Taking: A Standardized Measure for an Aspect of Stu- dents' Historical Thinking ${ }^{8}$}

There is ample consensus today that students studying history should do more than simply increase their factual knowledge about historical events, people and processes (e.g. Dickinson, Gordon \& Lee, 2001). Working with historical documents, evaluating contradictory evidence or taking historical perspectives contribute to students' historical thinking, empowering them to encounter various historical accounts in school, in everyday life and in the media.

However, making historical thinking a standard in education is no simple matter, as Dickinson et al. (2001) have pointed out. Conceptually, research, policy and teachers have to agree on relevant competencies of historical thinking. In this study, we focus on a competency we call historical perspective taking (HPT). It means knowing that certain historical agents or groups had particular perspectives on their world, and being able to see how that perspective would actually have affected actions in different situations (Lee \& Ashby, 2001). Students who achieve this will not only benefit in terms of historical understanding but will also be better able to cope with the present world (Ashby \& Lee, 1987). For these reasons HPT has become a standard in various models of historical thinking. In the United States, the National Standards for History in the Schools (National Center for History in the Schools, 1996) mention "appreciating historical perspectives" as one part of historical comprehension. In the UK, this competency can be found in History Standard 2 of the National Curriculum - "knowledge and understanding of events, people and changes in the past". In Germany, which is the context of the present study, taking historical perspectives is part of a taxonomy proposed by the Association of History Teachers (Sauer, 2006).

Setting up a standard like HPT requires measures to test whether large numbers of students meet it. Measures for students' historical competencies have to be different from merely fact-checking, multiple-choice items; they require theoretical elaboration

\footnotetext{
${ }^{8}$ Dieses Unterkapitel entspricht einer Publikation, die in der Zeitschrift Learning and Individual Differences - Journal of Psychology and Education erschienen ist (Hartmann \& Hasselhorn, 2008).
} 
combined with a sensible methodological approach. To date, reliable standardized tests are lacking. The aim of the present study is to introduce a measure for students' HPT competency.

\subsubsection{Theoretical Background}

Yeager and Foster (2001) conceptualize HPT as a complex process. They include an understanding of historical context and chronology, the analysis of historical evidence and interpretations, and the construction of a narrative framework in their definition of this competency. Portal (1987) conceptualizes historical empathy as a heuristic process which can stimulate other forms of historical thinking. He emphasizes the imaginative component of this concept. To Lee and Ashby (2001), who regard HPT or historical empathy as a predominantly cognitive task, it consists of knowing that certain historical agents or groups had particular perspectives on their world and of being able to see how that perspective would actually have affected actions in different situations. In our study, we apply their understanding of HPT.

A central theme in discussions on HPT has been the problem of presentism. Judging historical agents from only a present-oriented perspective is widely regarded as non-historical and thus as reflecting a low level of competency (Ashby \& Lee, 1987; Barton, 1996). HPT is about escaping one's own views and opinions in order to understand past actions and events; however, some scholars have questioned this demand as being idealistic (VanSledright, 2001; Wineburg, 1999). Psychological phenomena like epistemic egocentrism (Royzman, Wright Cassidy \& Baron, 2003), the failure to set aside one's own privileged knowledge when thinking about others, imply that thinking in a present-oriented way could correspond to a general pattern of human behavior. Still, the goal remains for students to acquire contextual historical empathy as outlined in Ashby and Lee's (1987) taxonomy. Seixas (1996) argues that despite the fact that we can never be perfectly non-presentist, negotiating the tension between past and present is essential for sophisticated historical thinking.

Another component that is less clear in the research is the role of the historical agent. Nonetheless, it could function as an intermediate stage between presentism and contextualization. Students confronted with historical situations tend to assign roles to historical agents, often in a stereotypical way (e.g. Ashby \& Lee, 1987; Bermúdez \& 
Jaramillo, 2001; Lee \& Ashby, 2001). In doing so, they are able to refer to roles or institutions they know from their own lives (e.g. the role of a father or that of a businessman); these they then use to explain thoughts and actions of people in the past. Such attempts do not entirely meet the demands of historically contextualized thinking, but could mark a step forward in decentring from one's own completely presentist view. Even though this phenomenon has been observed in the research, its exact role with respect to students' competencies remains unclear.

In our study we include present-oriented perspective taking (POP), the ability to show contextualized thinking (CONT), as well as the notion of thinking about the role of the historical agent (ROA) to measure students' HPT competency.

In search of a measure of students' HPT with respect to the aforementioned components, we have noticed a gap in research on historical thinking. Standardized instruments hardly exist. Most evidence relies on qualitative studies using interview techniques or group discussions to target HPT competency (e. g. Ashby \& Lee, 1987; Shemilt, 1987; Voss \& Wiley, 1997; Wineburg, 1991). While these methods are wellsuited for gathering in-depth information about students' thought processes, they are less adequate for gaining a representative picture of larger student samples required for standard-based assessments. Two measures using questionnaires are worthy of mention: Gehlbach's (2004a) draws on Ashby and Lee's (1987) stages of historical empathy. It contains statements (reflecting different stages) on why the Greeks divided themselves into social classes although they believed in a democratic government. Von Borries measured historical empathy as part of the Youth and History Study (Angvik \& von Borries, 1997). Students were asked to imagine themselves in the position of a teenager in the 15th century who was forced to marry someone he or she neither knew nor loved. The data displayed the constructs "obedience" and "resistance", but no actual historical empathy as the author concludes.

Our strategy in constructing a standardized measure for HPT was to draw on approaches from a related theoretical concept, namely that of social perspective taking (SPT). According to Johnson (1975), SPT is the ability to understand how a situation appears to another person and how that person will react cognitively and emotionally to the situation. In many respects SPT and HPT show similarities. Bermúdez and Jaramillo (2001) emphasize that in both cases students have to shift from a self-centred point of view to the other person's perspective and then co-ordinate both to build a mental representation of society. Gehlbach (2004b) considers SPT a situated construct whereby time 
is a constituent component. He argues that temporality (taking past, present or future perspectives) should influence students' outcome regarding this competency; however, empirical evidence on this aspect is still lacking. Selman (1980) proposed a developmental sequence of social perspective coordination starting with an undifferentiated and egocentric perspective (level 0) transforming into an in-depth and societal-symbolic perspective taking, in which personalities are conceptualized as systems with their own developmental histories (level 4). To connect this to historical thinking, taking the perspective of someone living in a different time inherently requires coordinating between past and present. In Selman's level 4 a societal dimension of perspective taking is addressed. Applied to historical contexts, perspective coordination should depend on the specific time period during which social interactions take place.

Despite the similarities between HPT and SPT, major differences do exist. HPT requires that one adopt the view of another person who acts in an entirely different situation, time and/ or culture. This makes HPT even more challenging. Secondly, the types of coordination in SPT and HPT cannot be easily compared. While coordination in SPT happens within an interpersonal situation with the intent of improving communication and solving interpersonal conflict (Selman, 1980; 2003), the purpose of HPT is to provide a more plausible explanation of a historical situation. The perspective taker and the target person never meet; one is the subject, the other the object of historical investigation.

Still, the two concepts seem to share sufficient commonality to use ideas of SPT in constructing a standardized measure for HPT. Various measures have been developed to assess students' SPT. One strand consists of video measures to assess accuracy of perspective taking (e.g. Gehlbach, 2004a; Ickes, 1997; Ickes, Stinson, Bissonnette \& Garcia, 1990). Applying them to a history classroom seems difficult in many respects since participants have to be assessed individually and historical situations have to be re-enacted to meet the demands of the subject. A second strand relies on self-reports, as does the subscale "Perspective Taking" (Davis, 1983b). This scale is easy to implement but does not actually target competency in taking perspectives; it simply reveals an intention to do so. A third strand comes from developmental research. To assess social perspective coordination, Selman (1980) conducted interviews with children using hypothetical interpersonal scenarios. This methodology has been modified according to theory (e.g. Yeates \& Selman, 1989), and has been transferred into written form (e.g. Gutzwiller-Helfenfinger, 2003; Mischo, 2005), also with rating-scale items (Hickey 
Schultz \& Selman, 2004). With regard to historical situations, it allows for the incorporation of rich contextual descriptions and is easily implemented in classroom settings.

\subsubsection{Research questions}

The aim of our study was to measure HPT with a new instrument that could be applied to large samples of students to assess whether they meet standards of historical thinking. Our intention was to measure three aspects of HPT: present-oriented perspective taking (POP), the role of the historical agent (ROA) and students' ability for historical contextualization (CONT). Our first goal was to examine the structure of our HPT measure. Second, we were interested in whether students could be reliably discerned according to their ability to take historical perspectives. A third issue was the criterion-related validity of the instrument. If students' specific HPT competency as measured by our instrument offers meaning for history education, it should relate to their history grade as an indicator of their performance in classroom.

\subsubsection{Method}

In search of a theory-based, context-related, and standardized measurement with high curricular validity, we decided to work with a hypothetical historical scenario and an item-rating format. We applied Selman's (1980) methodology, and modified it to assess a core competency of historical thinking. By this means, we could use a historical context that is integral part of the history curriculum. We refrained from using a primary or secondary source as a task for assessing HPT, since this would also require measuring indirectly students' competency to handle historical documents or other parts of historical inquiry. To ensure that our scenario reflected a historically plausible situation, it was reviewed by expert historians. We selected a scenario set in the Weimar Republic and investigated students attending $10^{\text {th }}$ grade where this topic is extensively covered. 


\section{Participants}

186 students from ten $10^{\text {th }}$ grades at three urban grammar schools (the top track in the German school system) participated in the study. 16 students were excluded because of missing data. This leaves 170 cases ( 71 male, 99 female) with a mean age of 16 years. $37 \%$ had immigrant status.

\section{Measurement}

Historical Perspective Taking: The scenario we developed presents a young man (Hannes) in the Weimar Republic in Germany, thinking about which party to vote for in the 1930 election (see A.1). He talks to a friend about the situation in Germany during that time. In a country that struggles with the legacy of World War 1 and a deep economic crisis, he prefers a leader who will bring back prosperity. Students were requested to take Hannes' perspective and decide whether he was likely to vote for an antidemocratic party like the NSDAP. Nine items were formulated which were based on the aforementioned aspects of historical perspective taking. Three items display presentoriented perspective taking (POP). Item POP2, for example, introduces a contemporary perspective, which is projected onto the historical agent in the scenario. Hannes is supposed to see that only in a democracy can people participate in decision-making. The second group of items contains statements which address a specific role of the historical agent in the scenario (ROA). In ROA3 Hannes is seen as the son of a desperate businessman whose company is in jeopardy, thus influencing his decision. Three items demonstrate historical contextualization (CONT). CONT1 points out that Hannes has had little democratic experience as this form of government had not yet prevailed; therefore, he is likely to vote for Hitler's party.

Items were shown in random order; students answered them on a four-point rating scale from 0 (doesn't fit his situation at all) to 3 (fits his situation very well). To assess face validity, four coders sorted the items into the three groups (POP, ROA, CONT). Inter-coder analyses revealed good consistency $(\kappa=.83)$.

History grade: Students were asked for the history grade from their last report card. Each grade was coded from 0 (unsatisfactory) to 15 (excellent) based on the German grade system. 
Procedure

The study took place as a group assessment in classrooms. Written and oral instructions were given to students before they worked on the scenario. All students succeeded in completing the task within 15 minutes.

\subsubsection{Results}

\section{Dimensionality of the HPT measure}

We applied a principal component analysis to examine the structure of our measure. Two factors were extracted which met the criteria of an eigenvalue $>1$ and Cattell's Scree Plot. They accounted for 51\% of the variance (factor 1: 35\%; factor 2: $16 \%)$. Results are presented in table 2 . The factor loadings after varimax rotation reveal that POP and CONT items constitute one factor representing the two poles of presentism and contextualization. Two ROA items (ROA2 and ROA3) constitute the second factor. One item in this category (ROA1) violates simple structure with loadings above .40 on both factors; it was consequently excluded from further analysis.

Table 2: Results of the principal component analysis

\begin{tabular}{lcc}
\hline Item & \multicolumn{2}{c}{ Factor loadings (rotated) } \\
& 1 & 2 \\
\hline POP1 & -.777 & .080 \\
POP2 & -.707 & .265 \\
POP3 & -.675 & .308 \\
ROA1 & .630 & .433 \\
ROA2 & -.142 & .755 \\
ROA3 & -.039 & .627 \\
CONT1 & .527 & -.003 \\
CONT2 & .592 & -.257 \\
CONT3 & .754 & .113 \\
\hline
\end{tabular}

Note. POP - present-oriented perspective-taking; ROA - role of the historical agent; CONT - historical contextualization. 


\section{Student classes of HPT competency}

To differentiate among students in this two-dimensional concept of historical perspective taking, we chose Latent Class Analysis (LCA) (Lazarsfeld \& Henry, 1968). The existing data were found to fit a rating scale model with class specific parameters. For selecting models in this study, the Bayesian Information Criterion (BIC), which accounts for sample size, was used. Additionally, we report results of the Consistent Akaike Information Criterion (CAIC). The model with the smallest BIC and CAIC values is chosen as the one which best represents the data.

One apparent problem in LCA - unless a sample size is enormous - is that the number of possible response patterns exceeds the number of participants. Thus, the chisquare $p$-value approximation for the goodness-of-fit statistics is not appropriate as a model selection criterion. One solution is to use a parametric bootstrap to calculate fit statistics (von Davier, 1997). We used Pearson and Cressie Read statistics to estimate model fit.

To gain information about the nature of each latent class, the average classspecific item scores were plotted against each item across the classes. This enabled us to examine response profiles and detect qualitative and quantitative differences in response patterns between subpopulations. We conducted LCA with the WINMIRA 2001 program (von Davier, 2001).

A latent class mode (LCM) with three classes best fits to the data (table 3), since both BIC and CAIC are smallest and the parametric bootstrap reveals values above .05 for Cressie Read and Pearson statistics.

Table 3: Results of the latent class analysis

Empirical $p$ value using paramet-

BIC CAIC ric bootstrap method

\begin{tabular}{lcccc} 
& & & Cressie Read & Pearson $\chi^{2}$ \\
\hline LCM with 1 class & 2984.49 & 2994.49 & 0.000 & 0.000 \\
LCM with 2 classes & 2832.21 & 2853.21 & 0.040 & 0.100 \\
LCM with 3 classes & 2809.39 & 2841.39 & 0.075 & 0.170 \\
LCM with 4 classes & 2833.64 & 2876.64 & 0.020 & 0.020 \\
\hline
\end{tabular}


Item score profiles for the three latent classes are plotted in figure 1. The largest class, containing $66 \%$ of the students, is characterized by moderately low scores in POP items and moderately high scores in CONT items. Class 2 yields a clearer pattern. POP items are strongly rejected whereas CONT items are strongly supported. Compared to the first class, the ROA items are answered a little more depreciatively. Class 3 shows a reverse response pattern with regard to POP and CONT items. POP items are moderately supported within this group but CONT items are moderately rejected. ROA items are answered in a moderate way with a relatively high score in ROA2 compared to classes 1 and 2. This class is also the smallest in size containing 10\% of the students. With respect to response patterns class 2 seems to perform high on HPT while classes 1 and 3 contain middle and low performing students.

As a measure for the reliability of this model, the expected class membership probabilities for the latent classes appear in table 4. High values in the diagonal indicate that students can be reliably assigned to the three classes of this model.

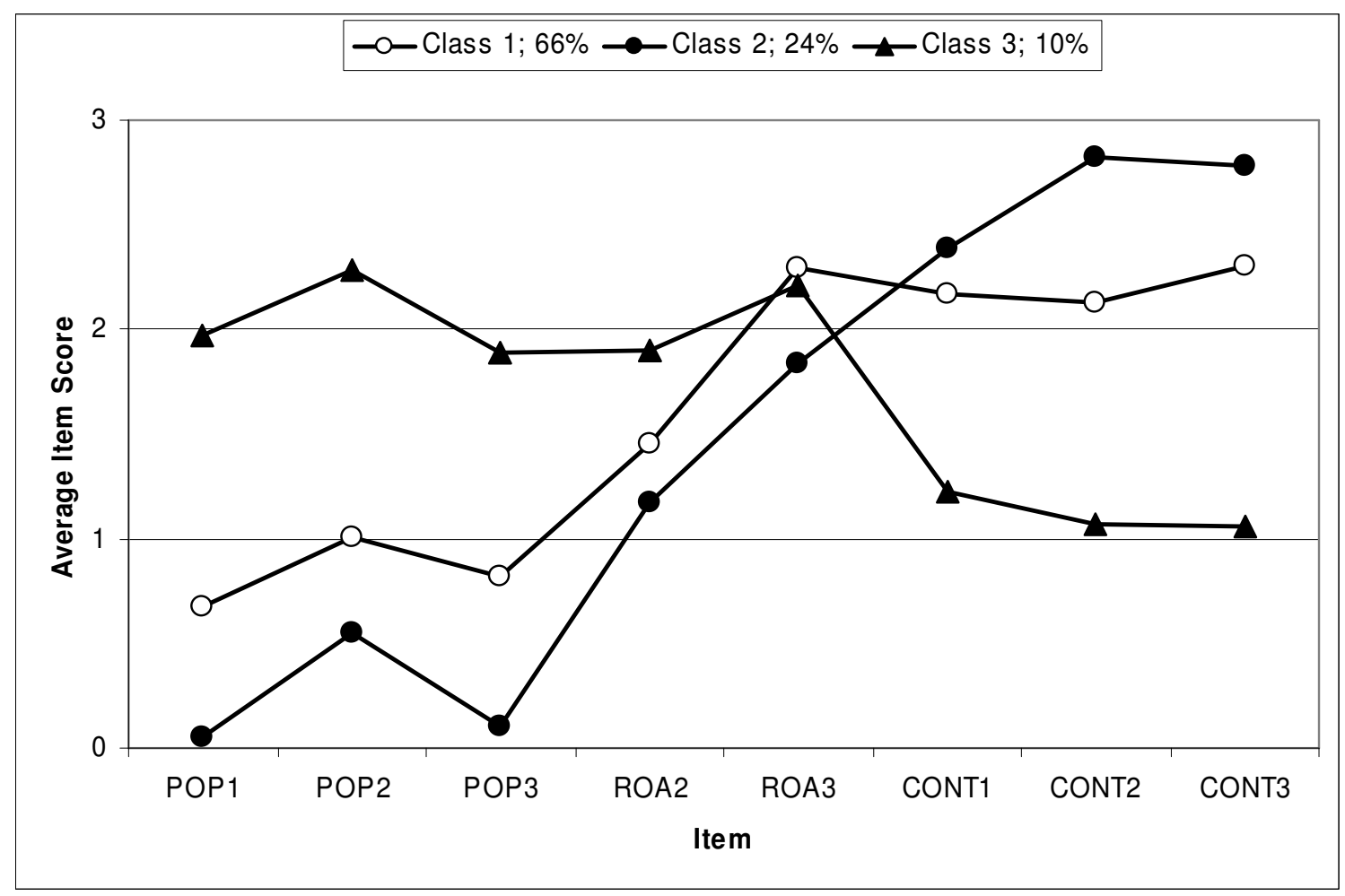

Figure 1: Item score profiles of the HPT measure 
Table 4: Expected class membership probabilities

\begin{tabular}{lccc}
\hline & Class 1 & Class 2 & Class 3 \\
\hline Class 1 & 0.938 & 0.046 & 0.015 \\
Class 2 & 0.127 & 0.872 & 0.000 \\
Class 3 & 0.004 & 0.000 & 0.996 \\
\hline
\end{tabular}

\section{Relation of students' historical perspective taking to history grade}

After selecting LCMs for students' historical perspective taking competency, HPT class membership - calculated for each student by LCA - was related to students' history grades. Core assumptions for conducting parametric tests were violated (e.g., classes of similar sizes and variances), so we used a Kruskal-Wallis test. The history grade was connected to the class assigned by the HPT measure $(H(2)=19.61, p<.001)$. We used Mann-Whitney tests to follow up this finding. Applying a Bonferroni correction, we established a 0.0167 level of significance. The history grade for members of class 1 was lower than for those of class $2(U=1457.5, r=-.29)$; it was higher for class 2 than for class 3 members $(U=104.5, r=-.51)$. Students who showed the highest agreement to the CONT items and the highest rejection to the POP items earned better history grades than did both other groups. It made no difference for history grade whether a student belonged to the middle (class 1) or the lowest (class 3) HPT class ( $U$ $=589, r=-.17)$.

\subsubsection{Discussion}

In this study we have introduced a standardized curriculum-related measure to assess students' competency in HPT. Incorporating research on historical thinking, we have identified three aspects of HPT, forming two dimensions of the measure. The first consists of the two poles presentism and contextualization; the second includes items about the role of the agent. Three student classes of HPT competency could be reliably distinguished. One class, which contains $10 \%$ of the sample, answers present-oriented items moderately positive but rejects items with historical contextualization. We assume that this pattern reflects low performance in HPT. The second class $(66 \%)$ shows a reverse pattern with moderately negative ratings on the POP items but moderately posi- 
tive ratings on CONT items. The third class (24\%) strongly rejects POP items but strongly agrees to CONT items. Supporting our impression that this reflects a high HPT competency relevant for history learning in schools, these students get better history grades than do the other two classes. We regard this finding as a first indication of the instrument's criterion-validity. However, we did not find a difference in history grades between low and middle HPT class, even though their response patterns look almost opposite to each other. Furthermore, the percentage of variance explained by the two factors is only $51 \%$. We can only speculate whether the remaining $49 \%$ are relevant HPT factors. It seems plausible to attribute them to historical content knowledge, or to attitudes which might contribute to students' performance as well.

We were also unable to clarify the implications of the ROA items. Students with better history grades answer them more dismissively than do the other classes but differences between classes are very small. Possibly, competent students are more cautious in agreeing to simpler categorizations of historical agents, concordant with Bermúdez and Jaramillo (2001). Further refinement of these items may be necessary, especially since only two of our items clearly loaded on the ROA factor.

Our study is limited by its selective sample, since we only investigated students from the highest track in the German school system. This could explain the small number of students falling into the low-performing class. In studying $10^{\text {th }}$ graders we limited ourselves by looking only at one historical context from the relatively recent past. To provide a curriculum-related measure, this seemed to be appropriate for our sample; nevertheless, important questions remain about how students take historical perspectives when more distant times or foreign cultures are considered.

As a general limitation of performance measures, the underlying reasoning processes which lead students to respond to the items in a certain way remain unclear. Studies using additional interview techniques could identify features of students' thinking when taking historical perspectives. Moreover, expert ratings of history teachers could help clarify how competent students are expected to respond to the measure. Another point of interest is the teacher's part itself. Researchers could investigate which instructional arrangements support historical contextualization in students' HPT.

In conclusion, our study shows that measuring a specific historical competency such as HPT with a standardized instrument has attained initial positive results regarding reliability and validity. As students' HPT relates to their success in history classes, we suggest that further refinement of measuring this and other competencies of histori- 
cal thinking through research has important implications for educational policy and those who implement it. 


\subsection{Kompetenzprofile historischer Perspektivenübernahme in Klasse $7^{9}$}

\subsubsection{Methode}

\section{Messinstrument}

Die Erfassung historischer Perspektivenübernahme erfolgte im Anschluss an die Präsentation eines lehrplanbezogenen fiktiven historischen Szenarios. Das Szenario für die 7. Klasse (vgl. A.2) ist im Mittelalter angesiedelt. Die 22-jährige Johanna ist die Tochter eines Freiherrn und soll mit einem ihr weitgehend unbekannten Mann verheiratet werden, der ihrem Stand entspricht. Als diese Hochzeitspläne scheitern, weil der vorgesehene Mann sich für eine wohlhabendere und standeshöhere Frau entschieden hat, beschließen ihre Eltern, Johanna in ein Kloster zu geben, damit sie dort in abgesicherten Verhältnissen leben kann.

Alle Schüler wurden angewiesen, das Szenario zunächst durchzulesen, sich anschließend in die handelnde Person hineinzuversetzen und ihre Zustimmung bzw. Ablehnung zu vierstufigen Items abzugeben (vgl. A.2). Diese Items sind den drei Aspekten historischer Perspektivenübernahme zugeordnet, die bereits in Abschnitt 4.1 ausführlich beschrieben wurden. Jeweils drei Items stehen für eine gegenwartsfixierte Betrachtung der historischen Situation (im Ergebnisteil mit GEG abgekürzt). Darin werden Maßstäbe der heutigen Zeit unreflektiert auf die im historischen Szenario handelnde Person übertragen. Die zweite Gruppe von Items beinhaltet eine Aussage über die Rolle des historisch Handelnden (abgekürzt mit ROLLE), diese Rolle ist jedoch noch eher ,zeitlos“, also noch nicht im spezifischen historischen Kontext verankert. In der dritten Itemgruppe wird der historische Kontext des Szenarios berücksichtigt und Unterschiede zwischen Gegenwart und Vergangenheit werden angesprochen (im Folgenden mit KONT abgekürzt).

\footnotetext{
${ }^{9}$ Dieses Unterkapitel ist die gekürzte und modifizierte Fassung eines Beitrags im Buch Forschungswerkstatt Geschichtsdidaktik empirisch 07 (Hartmann, im Druck).
} 


\section{Stichprobe und Durchführung}

Die Stichprobe der 7. Klasse bestand aus 110 Mädchen und 98 Jungen mit einem Altersdurchschnitt von 13 Jahren. Die Untersuchung fand an drei niedersächsischen Gymnasien statt. Die Befragung dauerte zwei Schulstunden. Die Messung historischer Perspektivenübernahme war der zentrale Aspekt der Untersuchung. Weiterhin wurden Variablen wie das Interesse und die Leistung im Fach Geschichte und die Fähigkeit zur sozialen Perspektivenkoordination (nach Selman, 1980) erhoben, auf die erst in Kapitel 5 eingegangen wird.

\section{Leitfaden für die Datenanalyse}

In einem ersten Schritt wurden die Daten einer explorativen Faktorenanalyse unterzogen. Ziel war es, die Datenstruktur zu untersuchen und festzustellen, ob sich die insgesamt neun Items sinnvoll den drei Kategorien GEG, ROLLE und KONT zuordnen ließen. Ungeeignete Items wurden aufgrund dieser Analysen ausgeschlossen.

Im zweiten Schritt wurden die verbleibenden Items mittels Latenter Klassenanalyse (LCA) ausgewertet. Basierend auf den Annahmen der probabilistischen Testtheorie ermöglicht dieses Verfahren die Erstellung von Kompetenzprofilen historischer Perspektivenübernahme, indem es Klassen von Schülern (so genannte latente Klassen) auffindet, deren Antwortmuster einander ähnlich sind. Mithilfe der LCA sind Aussagen über Anzahl und Größe dieser Klassen möglich sowie über die Wahrscheinlichkeit, mit der eine Person einer bestimmten Klasse angehört. Anschließend können die Antwortprofile inhaltlich analysiert werden um zentrale Charakteristika einer jeden latenten Klasse herauszuarbeiten. Für eine ausführlichere Darstellung des Verfahrens vgl. Abschnitt 4.1.4.

\subsubsection{Ergebnisse}

\section{Faktorenstruktur}

Die Daten der Siebtklässler wurden analog zu denen der Zehntklässler einer explorativen Faktorenanalyse unterzogen um Aussagen über die Struktur historischer Perspektivenübernahme machen zu können. Die Ergebnisse sind in Tabelle 5 darge- 
stellt. Der erste Faktor lässt sich als Gegenwartsfixierung interpretieren. Alle drei GEGItems laden hoch auf diesem Faktor, wobei das Muster des Items GEG1 etwas weniger klar ist. Der zweite Faktor entspricht der Rolle des historischen Akteurs. Zwei der drei ROLLE-Items weisen Ladungskoeffizienten über .80 auf. Der dritte Faktor kann als historische Kontextualisierung interpretiert werden. Item KONT2 weist jedoch keine Einfachstruktur auf und lädt substanziell negativ auf dem Faktor Gegenwartsfixierung. Da dieses Ladungsmuster jedoch den Daten der Zehntklässler entspricht (vgl. Tabelle 2), in denen Gegenwartsfixierung und historische Kontextualisierung einen gemeinsamen Faktor bilden, wird das Item KONT2 zunächst in der Analyse belassen. Ausgeschlossen werden lediglich GEG1 und ROLLE2.

Tabelle 5: Faktorenstruktur der Daten in Klasse 7

\begin{tabular}{lccc}
\hline Item & \multicolumn{3}{c}{ Faktorladungen (rotierte Lösung) } \\
& 1 & 2 & 3 \\
\hline GEG1 & .516 & -.227 & \\
GEG2 & .713 & .271 & \\
GEG3 & .760 & .132 & \\
ROLLE1 & & .851 & .491 \\
ROLLE2 & -.327 & & \\
ROLLE3 & & .852 & .740 \\
KONT1 & & -.174 & .294 \\
KONT2 & -.563 & .197 & .711 \\
KONT3 & & .122 & \\
\hline
\end{tabular}

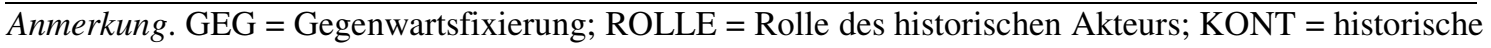
Kontextualisierung.

Profile historischer Perspektivenübernahme in Klasse 7

Nach Durchführung der Latenten Klassenanalyse lässt sich keine eindeutige Entscheidung für eins der Modelle fällen. Das 3-Klassenmodell weist verglichen mit dem 2- und dem 4-Klassenmodell die besten Werte für BIC und CAIC auf (vgl. Tabelle 6). Allerdings fallen die Bootstrap-Analysen nicht zufriedenstellend aus. Ausschlaggebend für die Wahl eines Modells sind bei einer solchen unsicheren Datenlage letztlich die Sparsamkeit sowie die Interpretierbarkeit der Modelle. Da das 3-Klassenmodell im 
Hinblick auf beide Kriterien überlegen ist, werden die Kompetenzprofile auf seiner Basis analysiert.

Tabelle 6: Vergleich verschiedener Latent Class Models (LCM)

\begin{tabular}{lcccc}
\hline & BIC & CAIC & \multicolumn{2}{l}{$\begin{array}{l}\text { Empirischer } p \text {-Wert nach } \\
\text { Bootstrap-Verfahren } \\
\text { Cressie Read }\end{array}$} \\
& & & Pearson $\chi^{2}$ \\
\hline LCM mit 2 Klassen & 3215.13 & 3230.13 & 0.000 & 0.015 \\
LCM mit 3 Klassen & 3191.71 & 3213.71 & 0.000 & 0.000 \\
LCM mit 4 Klassen & 3275.92 & 3309.92 & 0.062 & 0.062 \\
\hline
\end{tabular}

Anmerkung. Gewähltes Modell: Ratingskalenmodell mit klassenübergreifenden Schwellenparametern.

In Abbildung 2 sind die Antwortprofile der drei gefundenen Typen dargestellt. Auf der x-Achse befinden sich die einzelnen Items zu den Aspekten Gegenwartsfixierung (GEG), Rolle des historisch Handelnden (ROLLE) und historische Kontextualisierung $(\mathrm{KONT})^{10}$. Die y-Achse beschreibt die Zustimmung (hohe Werte) bzw. Ablehnung (geringe Werte) zu den einzelnen Items in jeder latenten Klasse.

Es lassen sich anhand dieser Grafik drei Typen von Schülern identifizieren, die sich in ihrer Beurteilung der Items unterscheiden. Dabei steht Typ 1 den gegenwartsfixierten Items neutral gegenüber. Items zur Rolle des historisch Handelnden werden zustimmend beantwortet und dem ersten Item zur historischen Kontextualisierung wird zugestimmt, das zweite wird neutral beantwortet.

Typ 2 steht den gegenwartsfixierten Items verglichen mit den beiden anderen Typen ablehnend gegenüber. Die Items zur Rolle des historisch Handelnden werden von diesem Schülertyp neutral beantwortet. Bei den Items zur historischen Kontextualisierung beantwortet dieser Schülertyp ebenfalls das erste zustimmender als das zweite, insgesamt jedoch etwas moderater als Typ 1.

Typ 3 weist in den gegenwartsfixierten Items ein anderes Muster auf. Ihnen wird klar zugestimmt. Auch die Items zur Rolle werden von diesem Schülertyp positiv bewertet, die Items zur historischen Kontextualisierung hingegen negativ.

\footnotetext{
${ }^{10}$ Für das letztendlich gewählte Modell (dargestellt in Abbildung 2) wurde das Item KONT1 ebenfalls entfernt, da sich hinsichtlich dieses Items keine substanziellen Unterschiede zwischen den drei latenten Klassen feststellen ließen.
} 
In Bezug auf die Kompetenz dieser Schülertypen erscheint Typ 3 am wenigsten in der Lage, die historische Perspektive einzunehmen. Zwischen Typ 1 und 2 fällt die Differenzierung noch schwer. Große Unterschiede gibt es in den Items zur Rolle, die auf den Gehorsam der Tochter gegenüber den Eltern abzielen. Es ist fraglich, ob mit dem Instrument an dieser Stelle nicht etwas anderes als die stereotype Betrachtung historischer Perspektiven gemessen wird, beispielsweise die Neigung zum Gehorsam der Schüler selbst. Beurteilt man die Typen nach der Beantwortung der KONT Items, erscheint Typ 1 etwas kompetenter als Typ 2. Allerdings ist die Gegenwartsfixierung bei Typ 1 ebenfalls etwas stärker ausgeprägt - ein Befund, der gegen die Einstufung als kompetenteste Gruppe spricht. Auffällig ist, dass keiner der drei Schülertypen dem letzten Item zustimmt, in dem es um die Erkenntnis geht, dass Religion im Mittelalter eine wichtigere Rolle gespielt hat als heute und dass Johannas Reaktion auf die elterliche Entscheidung sie ins Kloster zu geben nur vor diesem Hintergrund verstanden werden kann. Diese Einsicht ist bei einem großen Teil von Schülerinnen und Schülern der 7. Klasse noch nicht vorhanden.

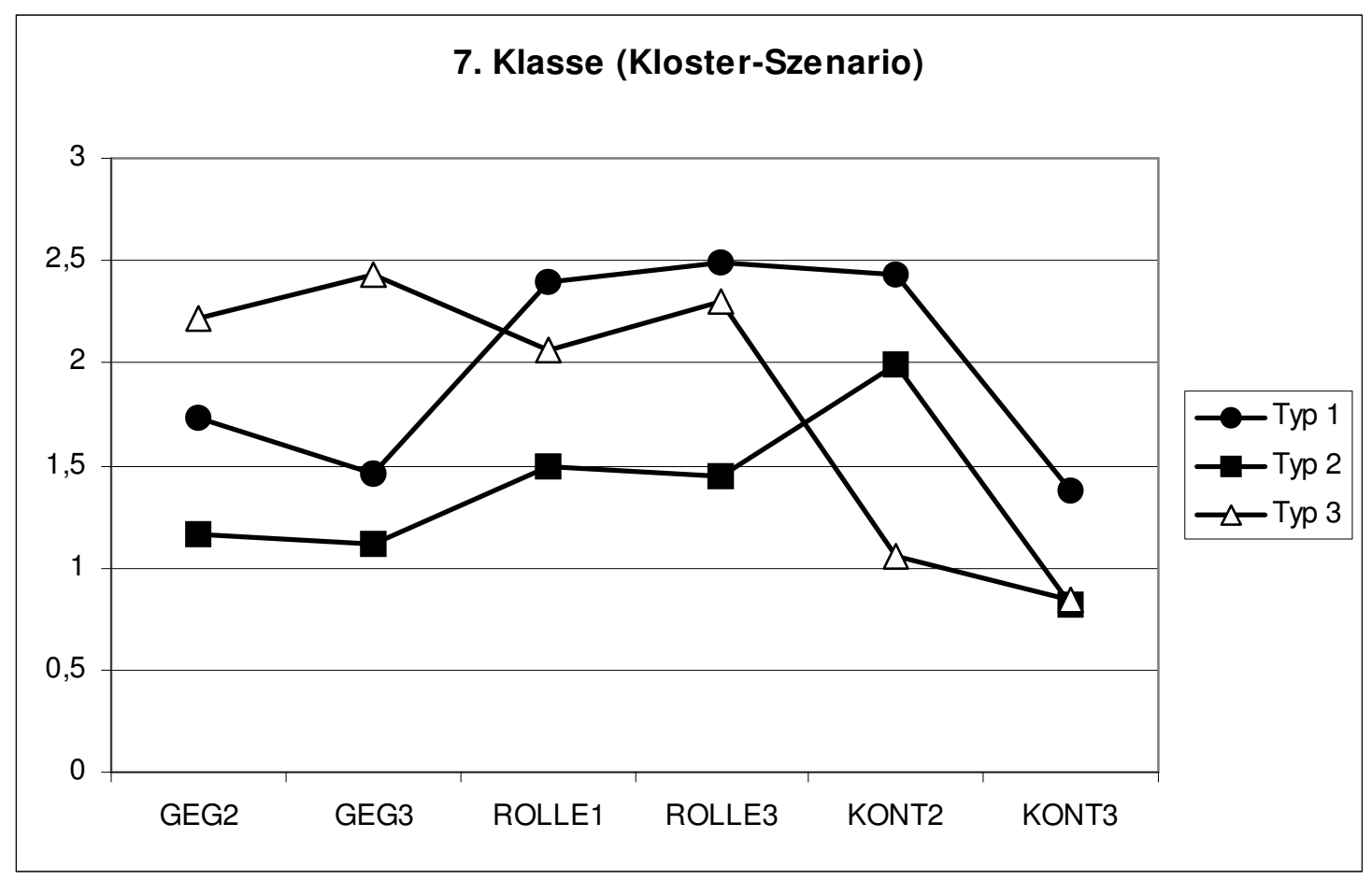

Abbildung 2: Kompetenzprofile in Klasse 7

Anmerkungen. 0 = starke Ablehnung, 3 = starke Zustimmung. Größe der latenten Klassen: Typ $1=47 \%$; Typ $2=31 \%$; Typ $3=23 \%$. 


\subsection{Vergleichende Zusammenfassung der Kompetenzprofile in Klasse 7 und $10^{11}$}

Die Ergebnisse dieses Kapitels liefern einen ersten Einblick in die Kompetenz von Schülern, historische Perspektiven einzunehmen. Mittels historisch plausibler, jedoch fiktiver Szenarien und einer Item-Rating-Methode lassen sich Schülertypen identifizieren, die sich durch eine eher gegenwartsfixierte oder durch eine den historischen Kontext berücksichtigende Perspektivenübernahme auszeichnen. Der Aspekt zur Rolle des historisch Handelnden bedarf weiterer Untersuchungen. Er erweist sich für Klasse 10 als wenig differenzierend. In Klasse 7 lassen sich zwar Unterschiede feststellen, diese sind jedoch nicht eindeutig interpretierbar im Sinne von höherer bzw. geringerer Kompetenz.

Ausgehend von diesen Befunden liegt die Interpretation nahe, dass in der 10. Klasse die Berücksichtigung des historischen Kontextes und die Ablehnung rein gegenwartsfixierter Items von ca. $25 \%$ der Schüler erreicht werden. In der 7. Klasse kristallisiert sich ein solcher Typ noch nicht heraus; gegenwartsfixierte Items werden von allen Befragten zustimmend bis allenfalls neutral bewertet.

Als Gründe für diese Unterschiede zwischen den Klassenstufen 7 und 10 muss neben den theoretisch plausiblen Entwicklungsfortschritten einer solchen Kompetenz eine methodische Schwierigkeit der Studie angesprochen werden. Die Unterschiedlichkeit der beiden historischen Kontexte (den Lehrplänen für die beiden Klassenstufen geschuldet) stellt eine Hürde in der Interpretation der vorliegenden Befunde dar. Auch wenn bei der Konstruktion der Szenarien auf vergleichbare Schwierigkeit der Texte und Items geachtet wurde, sind die Kontexte Mittelalter vs. Weimarer Republik nicht vergleichbar und bergen spezifische Herausforderungen bei der Übernahme der jeweiligen Perspektiven. So mag etwa die Rekonstruktion von Denk- und Handlungshorizonten anspruchsvoller für das ferne Mittelalter erscheinen, während das Nachvollziehen einer Wahlentscheidung für oder gegen die NSDAP eine zusätzliche moralische Auseinandersetzung verlangen könnte. Um diese methodische Klippe zu umschiffen, könnte man sich in zukünftigen Studien für eine Konstanthaltung der Szenarieninhalte entscheiden. In diesem Fall ist jedoch das unterschiedliche Niveau historischen Wissens, über welches die Schüler zu einem Thema bereits verfügen, eine methodische Herausforderung. Dies müsste ebenfalls konstant gehalten werden um Vergleiche bezüglich der histori-

\footnotetext{
${ }^{11}$ Dieses Unterkapitel basiert ebenfalls auf dem Beitrag im Buch Forschungswerkstatt Geschichtsdidaktik empirisch 07 (Hartmann, im Druck).
} 
schen Perspektivenübernahme anzustellen. Doch Schüler unterschiedlicher Klassenstufen verfügen aufgrund der stufenspezifischen Lehrpläne nicht über vergleichbares Hintergrundwissen zu denselben historischen Inhalten. Für zukünftige Studien könnte eine Realisierung beispielsweise so aussehen, dass verschiedene Altersgruppen zum gleichen historischen Themenbereich unterrichtet würden bevor ihre Kompetenz zur historischen Perspektivenübernahme an einem für beide Klassenstufen identischen Szenario gemessen würde. Somit könnte das spezifische historische Wissen allen Schülern einheitlich vermittelt werden, bevor sie sich in die historische Situation hineinversetzen sollen.

Die mangelnde Vergleichbarkeit der beiden historischen Kontexte, die in Klasse 7 und 10 eingesetzt wurden, ist nur eine von mehreren Grenzen der in dieser Arbeit realisierten empirischen Studie. Eine ausführliche Diskussion dieser Grenzen erfolgt im Rahmen der dritten Publikation am Ende von Kapitel 5 (Abschnitt 5.7). 


\section{Perspektivenübernahme als Kompetenz für den Geschichtsunter- richt: theoretische und empirische Zusammenhänge zwischen fach- spezifischen und sozial-kognitiven Schülermerkmalen ${ }^{12}$}

\subsection{Einleitung}

Seit Beginn der Debatte um Bildungsstandards (Klieme et al., 2003) sind zahlreiche Bestrebungen festzustellen, fachspezifische Kompetenzen für alle Schulfächer zu formulieren. Kompetenzen werden nach Weinert (2001) als kognitive Fähigkeiten und Fertigkeiten zur Lösung von Problemen bezeichnet, verbunden mit motivationalen, volitionalen und sozialen Bereitschaften, diese in variablen Situationen zu nutzen. Ausgehend von dieser Definition wurde damit begonnen, vor allem im Bereich der Mathematik, des Lesens, der Naturwissenschaften und der Fremdsprachen Kompetenzmodelle zu formulieren, empirisch erfassbar zu machen und in konkretes Unterrichtsgeschehen umzusetzen.

Da die Kompetenzdebatte die gesamte Schul- und Bildungslandschaft betrifft, sind die so genannten ,weicheren“ gesellschafts- und geisteswissenschaftlichen Schulfächer ebenfalls angesprochen und stehen vor einer umfassenden neuen Herausforderung (z.B. Tenorth, 2008): denn eine Tradition, Schulleistungen und Kompetenzen zum Beispiel im Fach Geschichte systematisch zu erfassen, besteht bisher nicht. Vielfältig sind die Schwierigkeiten, wenn es darum geht, die heuristischen Ziele historisches Denken (z.B. Seixas, 1996; Wineburg, 1999) oder Geschichtsbewusstsein (z.B. Pandel, 1987; von Borries, 1995) in einzelne Teilkompetenzen aufzuschlüsseln, diese von rein deklarativem Faktenwissen abzugrenzen und messbar zu machen. Dennoch haben in den letzten Jahren die wissenschaftlichen Bemühungen zugenommen, Kompetenzmodelle historischen Denkens zu formulieren. Im internationalen Raum gehen solche Modelle bereits in bildungspolitische Anforderungskataloge ein (z.B. die National Standards for History in den USA; National Center for History in the Schools, 1996). In Deutschland existieren momentan mehrere Kompetenzmodelle historischen Lernens nebeneinander. So wurde mit dem Modell der Gruppe FUER Geschichtsbewusstsein (z.B. Körber, Schreiber et al., 2007; Schreiber, 1999; 2008) eine Konzeption vorge-

\footnotetext{
${ }^{12}$ Dieses Kapitel entspricht einem Beitrag, der in der Zeitschrift für Erziehungswissenschaft erscheint (Hartmann, Sauer \& Hasselhorn, im Druck).
} 
schlagen, die historische Frage-, Orientierungs- und Methodenkompetenzen als konstituierend für historisches Denken ansieht. Pandel (2005) schlägt ein Modell mit den Bestandteilen Gattungskompetenz, Interpretationskompetenz, narrative Kompetenz und geschichtskulturelle Kompetenz vor. Sauer (2002; 2006) trennt dagegen Sachkompetenz, Deutungs- und Reflexionskompetenz und Medien-Methodenkompetenz voneinander, die wiederum in verschiedene Teilkompetenzen unterteilt werden. Sauers Annahmen stellen zudem die Grundlage für die Bildungsstandards des Verbandes der Geschichtslehrer Deutschlands (2006) dar. Bisher bleiben diese Ansätze weitgehend normativ, es liegen allenfalls erste empirische Befunde über die Ausprägungen von Schülerkompetenzen in den vorgeschlagenen Teilbereichen vor (z.B. Bernhardt 2007; Gautschi et al., 2007; Hartmann \& Hasselhorn, 2008; Martens, 2008).

Der Fokus der vorliegenden Studie liegt auf einer Teilkompetenz, die Bestandteil von geschichtsdidaktischen Modellen ist und damit als ein wichtiges Ziel historischen Lernens angesehen wird (z.B. Körber, Schreiber et al., 2007; National Center for History in the Schools, 1996; Sauer, 2002; 2006). Es handelt sich dabei um die Fähigkeit von Schülern, sich in historische Akteure und Situationen hineinzuversetzen und dabei die Lebensbedingungen sowie die Denk- und Handlungshorizonte der jeweiligen Zeit zu berücksichtigen. Diese Kompetenz umschreiben wir mit dem Begriff historische Perspektivenübernahme, womit die theoretische Anlehnung an das entwicklungspsychologisch begründete und erziehungswissenschaftlich relevante Konstrukt der sozialen Perspektivenübernahme angedeutet wird. In der Literatur wird historischer Perspektivenübernahme neben der Bedeutung für Leistungen im Geschichtsunterricht auch eine Rolle für sozial kompetentes Handeln in der Gegenwart zugeschrieben (z.B. Ashby \& Lee, 1987). Es existieren jedoch bisher keine empirischen Befunde zum Zusammenspiel historischer Perspektivenübernahme mit sozial-kognitiven und fachspezifischen Schülermerkmalen. Die vorliegende Studie liefert erste empirisch gestützte Hinweise zu dieser Schülerkompetenz für den Geschichtsunterricht. 
5.1.1 Perspektivenübernahme als Bindeglied zwischen sozialer Kompetenz und historischem Verstehen

Nach Johnsons (1975) Definition ist soziale Perspektivenübernahme die Fähigkeit zu verstehen, wie eine Situation sich für eine andere Person darstellt und wie diese Person kognitiv und emotional darauf reagiert. Die Zielperson ist dabei meistens ein Interaktionspartner, dessen Perspektive es zu verstehen gilt, und Perspektivenübernahme findet mit dem Ziel statt, Kommunikation zu verbessern und soziale Konflikte zu lösen (Selman, 1980; 2003; Yeates \& Selman, 1989). Soziale Perspektivenübernahme wird damit als ein integraler Bestandteil sozialer Kompetenz betrachtet (Caldarella \& Merell, 1997; Jerusalem \& Klein-Heßling, 2002; Selman, 2003). Definitionen von Perspektivenübernahme im Kontext des Faches Geschichte setzen einen anderen Schwerpunkt. Lee und Ashby (2001) ${ }^{13}$ verstehen das Konzept als ein Ergebnis einer historischen Rekonstruktionsleistung. Ihre Definition umfasst sowohl das Wissen, dass historische Akteure eine spezifische Perspektive auf ihre Welt hatten, als auch das Verständnis dafür, wie diese Perspektive die Handlungen dieser Akteure in bestimmten Situationen beeinflusst hat. Historische Perspektivenübernahme bedeutet also, die Handlungsergebnisse, die dem Historiker (oder dem Schüler im Geschichtsunterricht) beispielsweise durch Text- und Bildquellen zugänglich gemacht werden, historisch plausibel aus Intentionen, Motiven und Emotionen der Akteure abzuleiten und zu erklären. Dabei ist die Erkenntnis entscheidend, dass die Gedanken und das Verhalten historischer Akteure maßgeblich von Normen und Wertvorstellungen der jeweiligen Zeit und Gesellschaft mitbestimmt wurden. Bergmann (2000) beschreibt die Entwicklung von historischer Perspektivenübernahme bei Schülern als längeren Prozess, in dem zunächst eigene Erfahrungen und Lebensumstände auf frühere Zeiten übertragen werden. Nach und nach sind Schüler in der Lage, das Denken und Handeln früherer Menschen nachzuvollziehen und dabei auch frühere Lebensumstände einzubeziehen. Am Schluss dieses Prozesses können sie wahrnehmen, dass Menschen in ähnlichen Lebenslagen Unterschiedliches erreichen wollten und sich aufgrund ihrer Intentionen und Motive unterschiedlich ver-

\footnotetext{
${ }^{13}$ Lee und Ashby und einige weitere, vornehmlich britische Forscher verwenden anstatt des Begriffs historische Perspektivenübernahme den Begriff historical empathy. Sie beschreiben damit jedoch ebenfalls ein vornehmlich kognitives Konstrukt. Da der Empathiebegriff im Deutschen eher als eine emotionale Fähigkeit verstanden wird, haben wir uns dazu entschieden, durchgehend den Begriff historische Perspektivenübernahme zu verwenden, auch in Übereinstimmung mit Davis Jr. (2001). Zur Diskussion um die Begriffsvielfalt vgl. auch Baring (2004).
} 
halten haben. Historische Perspektivenübernahme entwickelt sich somit von einer gegenwartsfixierten Betrachtung historischer Situationen bis hin zur Berücksichtigung des historischen Kontextes und der dezidierten Wahrnehmung von Unterschieden zwischen Vergangenheit und Gegenwart. Downey (zit. nach Yeager \& Foster, 2001, S. 17) nimmt an, dass historische Perspektivenübernahme dann vorliegt, wenn Schüler realisieren, dass die Vergangenheit sich von der Gegenwart unterscheidet und dass historische Handlungsergebnisse aufgrund spezifischer zeitlicher und räumlicher Bedingungen zustande kamen.

Diese unterschiedlichen Zwecke von Perspektivenübernahme - auf der einen Seite das sozial kompetente Handeln, auf der anderen Seite die adäquate Rekonstruktion historischer Wahrnehmungs-, Denk- und Handlungshorizonte - führen jedoch nicht zu einer Unvereinbarkeit dieser beiden Konzepte. Ashby und Lee (1987) vermuten, dass das Einnehmen historischer Perspektiven ebenfalls die Bewältigung von Herausforderungen in der Gegenwart erleichtert. Gehlbach (2004a) nimmt an, dass die Fähigkeit zur Einnahme sozialer Perspektiven in alltäglichen Situationen ebenfalls die Fähigkeit begünstigen kann, sich in historische Akteure hineinzuversetzen. In einer Studie mit Neunt- und Zehntklässlern findet er moderate signifikante Zusammenhänge zwischen Maßen sozialer und historischer Perspektivenübernahme. Von Borries (1995) vermutet, dass ein Mangel an historischer Perspektivenübernahme das Risiko birgt, auch in der Gegenwart nicht erkennen zu können, wenn beispielsweise die Gefahr zum Mitläufertum besteht. Schüler gäben sich damit einer „Überlegenheitsillusion“ (von Borries, 1995, S. 388) gegenüber der Vergangenheit hin.

Einen Interventionsansatz, der historische Inhalte mit der Förderung von sozial kompetentem und gesellschaftlich verantwortungsvollem Handeln verknüpft, stellt das Unterrichtsprogramm „Facing History and Ourselves“ (FHAO) dar. In diesem Programm, welches vor allem an Schulen in den USA Bestandteil des Geschichts- oder Social Studies-Unterrichts ist, werden über die Beschäftigung mit Themen wie dem Holocaust Intergruppenkonflikte, moralische und ethische Fragen sowie Aspekte sozialer Gerechtigkeit und verantwortungsvollen Handelns in den Unterricht integriert. Die Förderung von sozialer Perspektivenübernahme ist ebenfalls zentraler Bestandteil dieses Programms. Eine Studie mit Achtklässlern, die an einem FHAO-Kurs teilnahmen, ergab Verbesserungen in der Koordination verschiedener Perspektiven bei gleichzeitiger Abnahme rassistischer Einstellungen sowie des selbstberichteten aggressiven Verhaltens (Hickey Schultz, Barr \& Selman, 2001). 
5.1.2 Zusammenhänge zwischen historischer Perspektivenübernahme, Motivation und Leistung im Geschichtsunterricht

Neben der Relevanz historischer Perspektivenübernahme für sozial kompetentes Verhalten von Schülern weist diese Kompetenz einen klaren fachspezifischen Bezug auf, wie es in der Expertise zu den Bildungsstandards (Klieme et al., 2003) gefordert ist. In der geschichtsdidaktischen Forschung wird Perspektivenübernahme als wichtiger Bestandteil des Unterrichtsfaches angesehen, erkennbar an zahlreichen Beiträgen (z.B. Baring, 2004; Davis Jr. et al., 2001; Portal, 1987; von Borries, 1995). Es ist jedoch bisher unklar, ob sich diese theoretisch vermutete Relevanz für das Fach auch empirisch zeigen lässt, d.h. ob historische Perspektivenübernahme beispielsweise mit der Leistung oder dem Interesse für Geschichte zusammenhängt. Auch zu den Zusammenhängen von Geschichtsleistung und Geschichtsinteresse untereinander existieren nur vereinzelte Beiträge. Es lohnt sich also der Blick auf andere Schulfächer, die bereits intensiver beforscht wurden. Beispielsweise finden Denissen, Zarrett und Eccles (2007) in ihrer Längsschnittstudie positive (und mit dem Alter in ihrer Stärke zunehmende) Zusammenhänge zwischen Leistung, Selbstkonzept und Interesse in den Bereichen Englisch, Mathematik und Naturwissenschaften. Diese scheinen auch im Fach Geschichte zu existieren (Rost \& Sparfeldt, 2002 für das Selbstkonzept sowie Kölbl, Tiedemann \& Billmann-Mahecha, 2006 für das Interesse). Es liegen jedoch ebenfalls Studien vor, die dezidiert auf die Unterschiedlichkeit der Fächer hinweisen. So berichten Stodolsky, Salk und Glaessner (1991) von deutlichen Unterschieden in Schülervorstellungen über Mathematik und Social Studies, und Wolters und Pintrich (1998) verweisen auf unterschiedliche Motivationslagen in Mathematik, Englisch und Social Studies.

Bei der Erforschung des Schulfachs Geschichte stehen insbesondere motivationale Komponenten wie das fachspezifische Interesse im Fokus. Gehlbach (2006) weist auf die Notwendigkeit hin, das Interesse am Geschichtsunterricht besonders zu fördern. Perspektivenübernahme könnte dazu ein Mittel sein, da das Hineinversetzen in andere Zeiten und Situationen diese etwas näher an die Lebenswelt der Schüler heranbringen könnte und die oftmals riesig erscheinende Kluft zwischen Vergangenheit und Gegenwart verringern könnte. Portal (1987) vermutet ebenfalls, dass die Kompetenz historische Perspektiven einzunehmen das Geschichtsinteresse von Schülern fördert. Kölbl et al. (2006) fanden in ihrer Untersuchung an Viertklässlern Zusammenhänge zwischen Interesse und Leistung im Fach Geschichte. Empirische Studien über den Zusammen- 
hang zwischen Teilkompetenzen historischen Lernens und dem Geschichtsinteresse existieren bisher jedoch nicht.

Zum Zusammenhang zwischen historischer Perspektivenübernahme und Leistung gibt es allenfalls erste theoretische Annahmen ohne entsprechende empirische Befunde. Davis Jr. (2001) sieht historisches Fachwissen als notwendige, jedoch nicht unbedingt hinreichende Voraussetzung für historische Perspektivenübernahme an. Auch Ashby und Lee (1987) betonen die Bedeutung von historischer Perspektivenübernahme für Leistungen im Geschichtsunterricht. Lee und Ashby (2001) weisen darauf hin, dass es erst historische Perspektivenübernahme sei, die es möglich mache von der Kenntnis historischer Fakten zum adäquaten Verständnis vergangener Handlungen zu gelangen.

Das Zusammenspiel von Teilkompetenzen historischen Lernens mit Indikatoren für fachspezifische Leistung und Motivation ist bisher noch nicht genauer untersucht worden. Gehlbach (2006) betrachtet Perspektivenübernahme, Interesse und Wissen in seiner Studie als abhängige Variablen, geht dabei aber nicht auf die Zusammenhänge zwischen ihnen ein.

\subsection{Ziel der Studie}

Die vorliegende Studie soll das bisher hauptsächlich theoretisch vermutete $\mathrm{Zu}$ sammenspiel zwischen der Schülerkompetenz historische Perspektivenübernahme, fachspezifischen Merkmalen sowie sozialer Perspektivenübernahme erstmals empirisch beleuchten. Da bisher nur sehr wenige empirische Studien zu dieser Teilkompetenz historischen Lernens vorliegen, wählen wir einen explorativen Zugang und betrachten $\mathrm{Zu}$ sammenhänge zwischen fachspezifischen Leistungs- und Motivationsindikatoren, der sozial-kognitiven Fähigkeit zur sozialen Perspektivenübernahme sowie einem neu entwickelten Maß für historische Perspektivenübernahme (Hartmann \& Hasselhorn, 2008; Hartmann et al., 2007). Als Leistungsindikatoren verwenden wir die Geschichtsnote sowie das Abschneiden in einem Lückentext zu historischen Themen, die zuvor im Unterricht behandelt wurden. Als Motivationsindikator untersuchen wir das Interesse im Fach Geschichte. Weiterhin nehmen wir das geschichtsbezogene Selbstkonzept in unsere Untersuchung auf, welches nach Rost und Sparfeldt (2002) sowohl Beziehungen zur Leistung als auch zum Interesse aufweisen sollte. Aufseiten der sozial-kognitiven Schülermerkmale untersuchen wir sowohl die Fähigkeit von Schülern, anhand eines fiktiven 
Szenarios soziale Perspektiven einzunehmen, als auch ihre Bereitschaft, diese Fähigkeit im Alltag zu zeigen.

Wir vermuten erstens, dass sich die bereits aus anderen Schulfächern bekannten und auch für das Fach Geschichte vereinzelt berichteten Zusammenhänge zwischen Schulnote, Selbstkonzept und Interesse auch in unserer Studie zeigen. Zweitens halten wir es für plausibel, dass positive Zusammenhänge zwischen der spezifischen Teilkompetenz historische Perspektivenübernahme und fachspezifischen Leistungs- und Motivationsindikatoren bestehen. Drittens halten wir aufgrund der theoretischen Nähe der beiden Konstrukte positive Zusammenhänge zwischen sozialer und historischer Perspektivenübernahme für denkbar. In diesem noch weitgehend unbearbeiteten Forschungsfeld erscheint uns die Formulierung spezifischer gerichteter Hypothesen zum jetzigen Zeitpunkt jedoch verfrüht. Vielmehr soll diese Studie dazu dienen, einen ersten empirischen Einblick in das Zusammenspiel fachspezifischer und sozial-kognitiver Schülermerkmale sowie einer Teilkompetenz historischen Denkens zu erhalten, auf deren Basis die Formulierung prüfbarer Hypothesen erst möglich wird.

\subsection{Methode}

\subsubsection{Stichprobe}

Die Daten der vorliegenden Studie entstammen einer Fragebogenerhebung an drei niedersächsischen Gymnasien. Es wurden alle 7. und 10. Klassen (insgesamt 375 SchülerInnen) befragt. 208 Personen (110 weiblich) mit einem Altersdurchschnitt von $M=13.03$ Jahren besuchten zum Zeitpunkt der Untersuchung die 7. Klasse. 167 Personen (97 weiblich) mit einem Altersdurchschnitt von $M=16.13$ Jahren entstammten der 10. Klasse. 38\% der Stichprobe wiesen einen Migrationshintergrund auf (entweder Schüler oder mindestens ein Elternteil im Ausland geboren). 


\subsubsection{Messinstrumente}

Im Folgenden sind die in der Studie eingesetzten Messinstrumente aufgeführt, auf die sich die berichteten Ergebnisse beziehen. Tabelle 7 informiert über die Kennwerte zur internen Konsistenz der Skalen getrennt für beide Klassenstufen.

Tabelle 7: Interne Konsistenzen der eingesetzten Messinstrumente

\begin{tabular}{|c|c|c|c|}
\hline & Skala & Cronbachs Alpha & Anzahl der Items \\
\hline \multirow[t]{7}{*}{$\begin{array}{l}\text { 7. Klasse } \\
(N=208)\end{array}$} & $\begin{array}{l}\text { Historische PÜ } \\
\text { (Kloster) }\end{array}$ & .51 & 6 \\
\hline & $\begin{array}{l}\text { Fähigkeit soziale Per- } \\
\text { spektivenkoordination }\end{array}$ & .85 & 9 \\
\hline & $\begin{array}{l}\text { Soziale PÜ } \\
\text { (Selbstbericht) }\end{array}$ & .74 & 5 \\
\hline & Interesse Geschichte & .65 & 2 \\
\hline & $\begin{array}{l}\text { Selbstkonzept } \\
\text { Geschichte }\end{array}$ & .85 & 8 \\
\hline & $\begin{array}{l}\text { Lückentext } \\
\text { „Mittelalter“ }\end{array}$ & .61 & 9 \\
\hline & Verbalskala KFT & .68 & 25 \\
\hline \multirow[t]{7}{*}{$\begin{array}{l}\text { 10. Klasse } \\
(N=167)\end{array}$} & $\begin{array}{l}\text { Historische PÜ } \\
(W a h l)\end{array}$ & .78 & 6 \\
\hline & $\begin{array}{l}\text { Fähigkeit soziale Per- } \\
\text { spektivenkoordination }\end{array}$ & .85 & 9 \\
\hline & $\begin{array}{l}\text { Soziale PÜ } \\
\text { (Selbstbericht) }\end{array}$ & .76 & 5 \\
\hline & Interesse Geschichte & .73 & 2 \\
\hline & $\begin{array}{l}\text { Selbstkonzept } \\
\text { Geschichte }\end{array}$ & .89 & 8 \\
\hline & $\begin{array}{l}\text { Lückentext } \\
\text { „Weimar/2. WK“ }\end{array}$ & .76 & 13 \\
\hline & Verbalskala KFT & .70 & 25 \\
\hline
\end{tabular}

Anmerkung. PÜ: Perspektivenübernahme. 


\section{Historische Perspektivenübernahme}

Die Erfassung historischer Perspektivenübernahme erfolgte im Anschluss an die Präsentation eines lehrplanbezogenen fiktiven Szenarios, in dem eine historische Person handelt. Alle Schüler sollten sich das Szenario zunächst durchlesen. Sie wurden instruiert, sich in die handelnde Person hineinzuversetzen und ihre Zustimmung oder Ablehnung zu vierstufigen Items abzugeben, von denen jeweils drei eine gegenwartsfixierte Betrachtung der historischen Situation implizieren und drei weitere den historischen Kontext und Unterschiede zwischen Gegenwart und Vergangenheit berücksichtigen.

Das Szenario der 7. Klasse (Kloster) ist im Mittelalter angesiedelt. Die 22jährige Johanna ist die Tochter eines Freiherrn und soll mit einem ihr weitgehend unbekannten Mann verheiratet werden, der ihrem Stand entspricht. Als diese Hochzeitspläne scheitern, weil der vorgesehene Mann sich für eine wohlhabendere und standeshöhere Frau entschieden hat, beschließen ihre Eltern, Johanna in ein Kloster zu geben, damit sie dort finanziell abgesichert leben kann. Die Schüler sollten einschätzen, was es für Johanna bedeutet, in ein Kloster geschickt zu werden. Der vollständige Text ist abgedruckt bei Hartmann et al. (2007). Das Szenario der 10. Klasse (Wahl) handelt von Hannes, dem Sohn eines bürgerlichen Schuhfabrikanten zur Zeit der Weimarer Republik. Die Firma seines Vaters steht vor dem Aus. Hannes ist verzweifelt über den $\mathrm{Zu}$ stand seines Landes und die wirtschaftlichen und sozialen Probleme und überlegt, welcher Partei er bei der kommenden Reichstagswahl seine Stimme geben soll. Politisch steht Hannes der DNVP nahe, fragt sich aber, ob deren Führungspersönlichkeiten einflussreich genug sind um die Krise abzuwenden. Die Schüler sollen einschätzen, ob Hannes seine Stimme einer antidemokratischen Partei wie der NSDAP geben wird. Eine ausführliche Beschreibung dieses Szenarios findet sich bei Hartmann und Hasselhorn (2008). Die Items zu beiden Szenarien sind in Tabelle 8 abgebildet.

Zur Skalenbildung wurden die jeweils sechs Items jedes Szenarios Faktorenund Korrelationsanalysen unterzogen. In den Daten der 10. Klasse konnte für diese Items eine klare einfaktorielle Struktur nachgewiesen werden mit den Polen Gegenwartsfixierung und Berücksichtigung des historischen Kontextes (vgl. auch Hartmann \& Hasselhorn, 2008). Die beiden Itemgruppen korrelieren untereinander mit $r=-.60, p<$ .01). Für die Daten der 7. Klasse lassen sich zwei Faktoren extrahieren. Dabei entfallen jedoch nur zwei Items der Gruppe Berücksichtigung des historischen Kontextes (K1 und K3) auf den zweiten Faktor. Das dritte Item dieser Gruppe (K2) lädt substanziell negativ auf dem ersten Faktor. Auch K1 und K3 weisen zwar geringe, aber negative Ladungen 
auf dem ersten Faktor auf. Es besteht auch in dieser Klassenstufe eine substanzielle negative Korrelation zwischen den Gruppen Gegenwartsfixierung und Berücksichtigung des historischen Kontextes $(r=-.27, p<.01)$. Aufgrund dieser offensichtlichen Beziehung zwischen den beiden Itemgruppen haben wir trotz der weniger eindeutigen Datenlage in Klasse 7 jeweils einen Skalenwert für historische Perspektivenübernahme gebildet. Die Items zur Gegenwartsfixierung sind umkodiert, sodass hohe Werte für hohe historische Perspektivenübernahmekompetenz stehen. Die interne Konsistenz ist in der 10. Klasse gut $(\alpha=.78)$ und in der 7. Klasse für die Zwecke dieser Studie noch ausreichend $(\alpha=.51)$.

Tabelle 8: Items zur Erfassung historischer Perspektivenübernahme

\begin{tabular}{|c|c|c|}
\hline & 7. Klasse (Kloster-Szenario) & 10. Klasse (Wahl-Szenario) \\
\hline \multirow[t]{3}{*}{$\begin{array}{l}\text { Gegen- } \\
\text { warts- } \\
\text { fixierung } \\
\text { (GEG) }\end{array}$} & $\begin{array}{l}\text { (GEG1) Für Johanna muss es furchtbar } \\
\text { sein, ins Kloster zu gehen, weil sie } \\
\text { dann keine Chance mehr hat, jemanden } \\
\text { kennen zu lernen, den sie liebt. }\end{array}$ & $\begin{array}{l}\text { (GEG1) Die NSDAP wird er bestimmt } \\
\text { nicht wählen. Was die mit Deutschland } \\
\text { und der Welt angerichtet haben, darf } \\
\text { man nicht zulassen. }\end{array}$ \\
\hline & $\begin{array}{l}\text { (GEG2) Sie wird ihre Eltern grausam } \\
\text { finden, weil sie sie in ein Kloster ge- } \\
\text { ben und ihr keine Möglichkeit geben } \\
\text { zu heiraten. }\end{array}$ & $\begin{array}{l}\text { (GEG2) Er wird merken, dass Men- } \\
\text { schen nur in einer Demokratie mit- } \\
\text { bestimmen können. Darum wird er } \\
\text { eine vernünftige Entscheidung treffen } \\
\text { und die heißt bestimmt nicht NSDAP. }\end{array}$ \\
\hline & $\begin{array}{l}\text { (GEG3) Johanna wird sich von ihren } \\
\text { Eltern schlecht behandelt fühlen, weil } \\
\text { sie ihr nicht ermöglichen, ein eigenes, } \\
\text { selbstbestimmtes Leben zu führen. }\end{array}$ & $\begin{array}{l}\text { (GEG3) Ich glaube nicht, dass er die } \\
\text { NSDAP wählt. Deren Parolen sind } \\
\text { doch leicht zu durchschauen und es ist } \\
\text { klar, dass das auf Krieg hinausläuft. }\end{array}$ \\
\hline \multirow{5}{*}{$\begin{array}{l}\text { Berück- } \\
\text { sichti- } \\
\text { gung des } \\
\text { histori- } \\
\text { schen } \\
\text { Kontex- } \\
\text { tes } \\
\text { (KONT) }\end{array}$} & $\begin{array}{l}\text { (KONT1) Auf die Heirat zu verzichten } \\
\text { ist für Johanna nicht so schlimm. Für } \\
\text { sie ist vor allem wichtig, dass sie für } \\
\text { ihr Leben versorgt ist. }\end{array}$ & $\begin{array}{l}\text { (KONT1) Hannes fehlt die Erfahrung } \\
\text { mit der Demokratie. Er weiß nicht, } \\
\text { welches Risiko die NSDAP bedeutet } \\
\text { und wird sie wahrscheinlich wählen. }\end{array}$ \\
\hline & (KONT2) Johanna weiß, dass ihre & (KONT2) Für ihn stellt Hitler einen \\
\hline & Eltern sie mit guten Absichten ins & starken Führer dar, der zeigt, wo es \\
\hline & $\begin{array}{l}\text { Kloster geben, damit sie auch als un- } \\
\text { verheiratete Frau versorgt ist. }\end{array}$ & $\begin{array}{l}\text { langgeht. An die Gefahren, die von der } \\
\text { NSDAP ausgehen, denkt Hannes eher } \\
\text { nicht. }\end{array}$ \\
\hline & $\begin{array}{l}\text { (KONT3) Religion spielt für Johanna } \\
\text { eine ganz andere Rolle als für viele } \\
\text { Menschen heute. Deshalb ist es für sie } \\
\text { sinnvoll, ein Leben im Kloster zu füh- } \\
\text { ren. }\end{array}$ & $\begin{array}{l}\text { (KONT3) In seiner Situation sieht er } \\
\text { nur Nachteile der Demokratie. Deshalb } \\
\text { werden ihn die Parolen der NSDAP } \\
\text { ansprechen. }\end{array}$ \\
\hline
\end{tabular}




\section{Fähigkeit zur sozialen Perspektivenkoordination}

Die soziale Perspektivenkoordination wurde in Anlehnung an Selman (1980) ebenfalls mit Hilfe von fiktiven sozialen Situationen erfasst. Es wurde das Freundschafts-Dilemma aus dem deutschen Written Interpersonal Understanding Interview von Gutzwiller-Helfenfinger (2003) eingesetzt (vgl. A.3). Im Original besteht dieses Instrument aus 15 offenen Fragen (z.B. „Was ist in dieser Geschichte das Problem? Warum ist das ein Problem?“), die sich an die Präsentation des Dilemmas anschließen. Für die vorliegende Studie wurden nur die ersten neun Fragen eingesetzt, da in diesen bereits alle Aspekte des Kodierschemas enthalten sind. 30\% der Schülerantworten wurden durch zwei unabhängige Rater in Bezug auf das Level der Perspektivenkoordination kodiert, die eine prozentuale Übereinstimmung von 74\% erreichten (Cohens $\kappa=.53$ ). Dies ist zwar nur zufriedenstellend, entspricht jedoch den bei Gutzwiller-Helfenfinger (2003) erzielten Werten und erscheint nach den Ausführungen von Wirtz und Caspar (2002) noch annehmbar, da es sich bei sozialer Perspektivenübernahme um ein relativ komplexes Konstrukt sozialer Kompetenz handelt.

\section{Soziale Perspektivenübernahme (Selbstbericht)}

Die Schüler wurden nach ihrer Bereitschaft gefragt, im Alltag Perspektiven anderer Personen zu übernehmen. Dazu wurde die Skala Perspective Taking (Davis, 1983b) in der deutschen Übersetzung eingesetzt (Kunter et al., 2002). Items wie „Bevor ich Leute kritisiere, versuche ich mir vorzustellen, wie es mir ginge, wenn ich an ihrer Stelle wäre“ wurden von den Schülern auf einer vierstufigen Skala (1 = trifft gar nicht $\mathrm{zu} ; 4$ = trifft völlig zu) beantwortet (vgl. A.3).

\section{Interesse an Geschichte}

Das Geschichtsinteresse wurde über zwei Items erfasst, die aus der PISASkalendokumentation (Kunter et al., 2002) entnommen und für das Fach Geschichte angepasst wurden. Die Items lauteten „Mir macht Geschichtsunterricht Spaß“ und „Ich lerne in Geschichte etwas, das für mich sehr wichtig ist" und wurden ebenfalls auf einer vierstufigen Skala ( 1 = trifft gar nicht zu; 4 = trifft völlig $\mathrm{zu})$ beantwortet. 
Fachspezifisches Selbstkonzept Geschichte

Die acht Items zum fachspezifischen Selbstkonzept wurden aus dem DISKGitter von Rost und Sparfeldt (2002) übernommen. Aussagen wie „Ich gehöre in Geschichte zu den Guten“"wurden von den Schüler ebenfalls auf einer vierstufigen Skala (1 = trifft gar nicht zu; 4 = trifft völlig zu) beantwortet (vgl. A.3).

Kenntnis zentraler historischer Fakten und Zusammenhänge

In beiden Klassenstufen wurde zur Überprüfung des Vorwissens ein Lückentext eingesetzt, der sich am Thema des historischen Szenarios orientierte und die Kenntnis zentraler Fakten und Zusammenhänge erfasste. In der 7. Klasse gab es neun Lücken zum Thema Mittelalter auszufüllen (vgl. A.4), in der 10. Klasse 13 Lücken zum Thema Weimarer Republik/ 2. Weltkrieg (vgl. A.5).

Geschichtsnote ${ }^{14}$

Die Schüler wurden nach ihrer letzten Zeugnisnote im Fach Geschichte gefragt. Die Antworten wurden nach dem Punktesystem für Schulnoten kodiert (0 bis 15 Punkte).

\section{Verbale Intelligenz}

Eine mögliche Artefaktquelle in dem hier gewählten Vorgehen zur Erfassung historischer Perspektivenübernahme besteht darin, dass die kompetente Bearbeitung der Szenarien allein die sprachlichen Fähigkeiten der Schüler widerspiegelt. Um dies zu kontrollieren, wurde die Verbale Intelligenz als zusätzliche Kontrollvariable eingesetzt und durch die Verbalskala V1 des Kognitiven Fähigkeitstests (KFT; Heller \& Perleth, 2000) erfasst. Maximal waren 25 Punkte zu erreichen.

\footnotetext{
${ }^{14}$ Die Teilstichprobe der 10. Klasse ist weitgehend identisch mit der bei Hartmann \& Hasselhorn (2008). Dort wurde bereits ein Zusammenhang zwischen historischer PÜ und der Geschichtsnote berichtet. Die Berechnung der sozialen Perspektivenübernahme beruhte bei Hartmann \& Hasselhorn jedoch auf der latenten Klassenanalyse (LCA), in die zusätzlich ein weiterer Aspekt - die Rolle des historisch Handelnden - einfloss. In der vorliegenden Studie wird die Geschichtsnote vor dem Hintergrund der aktuellen Fragestellungen erneut in die Analyse dieser Daten mit aufgenommen, da sie als Indikator für Schulerfolg neben den in dieser Studie erstmals analysierten Variablen Interesse, Selbstkonzept und dem Abschneiden im Lückentext eine wichtige Rolle spielt.
} 


\subsection{Ergebnisse}

In Tabelle 9 sind die Mittelwerte und Standardabweichungen der untersuchten Variablen dargestellt. Die Mittelwerte der Zehntklässler liegen bezogen auf fast alle erhobenen Variablen etwas über dem Niveau der Siebtklässler. Nur das Selbstkonzept in Geschichte und die selbstberichtete Bereitschaft zur sozialen Perspektivenübernahme weisen in beiden Klassenstufen ähnliche Durchschnittswerte auf. Die Kompetenz zur historischen Perspektivenübernahme (erhoben am Kloster-Szenario) ist in der 7. Klasse um ca. einen Skalenpunkt geringer ausgeprägt als die am Wahl-Szenario erhobene Kompetenz der Zehntklässler.

Tabelle 9: Deskriptive Statistiken zu den eingesetzten Skalen

\begin{tabular}{|c|c|c|c|}
\hline & Variable & $M$ & $S D$ \\
\hline 7. Klasse & historische PÜ & 2.15 & .44 \\
\hline \multirow[t]{8}{*}{$(N=208)$} & Geschichtsnote & 8.66 & 2.88 \\
\hline & Interesse an Geschichte & 2.43 & .78 \\
\hline & Selbstkonzept Geschichte & 2.53 & .56 \\
\hline & Summe Lückentext Mittelalter & 3.64 & 1.83 \\
\hline & KFT Untertest V1 & 15.86 & 3.55 \\
\hline & Fähigkeit soziale Perspektiven- & 2.28 & .18 \\
\hline & koordination & & \\
\hline & soziale PÜ (Selbstbericht) & 2.69 & .58 \\
\hline \multirow{10}{*}{$\begin{array}{l}\text { 10. Klasse } \\
(N=167)\end{array}$} & historische PÜ & 3.19 & .52 \\
\hline & Geschichtsnote & 8.37 & 2.99 \\
\hline & Interesse an Geschichte & 2.82 & .81 \\
\hline & Selbstkonzept Geschichte & 2.53 & .65 \\
\hline & Lückentext Weimar/ 2. Welt- & 6.53 & 3.02 \\
\hline & krieg & & \\
\hline & KFT Untertest V1 & 16.28 & 3.68 \\
\hline & Fähigkeit soziale Perspektiven- & 2.38 & .18 \\
\hline & koordination & & \\
\hline & soziale PÜ (Selbstbericht) & 2.77 & .57 \\
\hline
\end{tabular}

Anmerkung. PÜ: Perspektivenübernahme. 
Die Angaben der SchülerInnen zur Geschichtsnote wurden vor den weiteren Analysen einer klassenspezifischen z-Transformation unterzogen, sodass in jeder befragten Schulklasse ein Mittelwert von 0 und eine Standardabweichung von 1 vorlagen. Dies diente dazu, die Schulnoten, die aufgrund unterschiedlicher sozialer Bezugsnormen in den einzelnen Klassen zustande kamen, über Schulklassen hinweg vergleichbar zu machen. Unter dieser Voraussetzung halten wir es für gerechtfertigt, die Werte aus mehreren Schulklassen in einer Analyse zu berücksichtigen.

Es wurden Korrelationen zwischen der Kompetenz zur historischen Perspektivenübernahme, den geschichtsspezifischen und den sozial-kognitiven Indikatoren sowie der Verbalskala des KFT berechnet. In Tabelle 10 sind die Ergebnisse der 7. Klassenstufe aufgeführt. Die Geschichtsnote, das Interesse und das fachspezifische Selbstkonzept hängen signifikant positiv miteinander zusammen. Interesse und Geschichtsnote korrelieren zu $r=.21$. Das Selbstkonzept korreliert $\mathrm{zu} r=.56$ sowohl mit der Geschichtsnote als auch mit dem Interesse an Geschichte. Die Kompetenz zur historischen Perspektivenübernahme korreliert in dieser Klassenstufe hochsignifikant mit der Kompetenz zur sozialen Perspektivenübernahme $(r=.20)$. Weiterhin besteht ein signifikanter Zusammenhang zur Bereitschaft der Schüler, im Alltag Perspektiven anderer zu übernehmen $(r=.14)$. Dieses Selbstberichtsmaß zur sozialen Perspektivenübernahme korreliert außerdem mit dem Interesse $(r=.23)$ und dem Selbstkonzept in Geschichte $(r=.22)$. Weder in Bezug auf die fachspezifischen Motivations- und Leistungsvariablen noch den Untertest des KFT bestehen signifikante Korrelationen mit historischer Perspektivenübernahme. 
Tabelle 10: Korrelationen, Klassenstufe 7

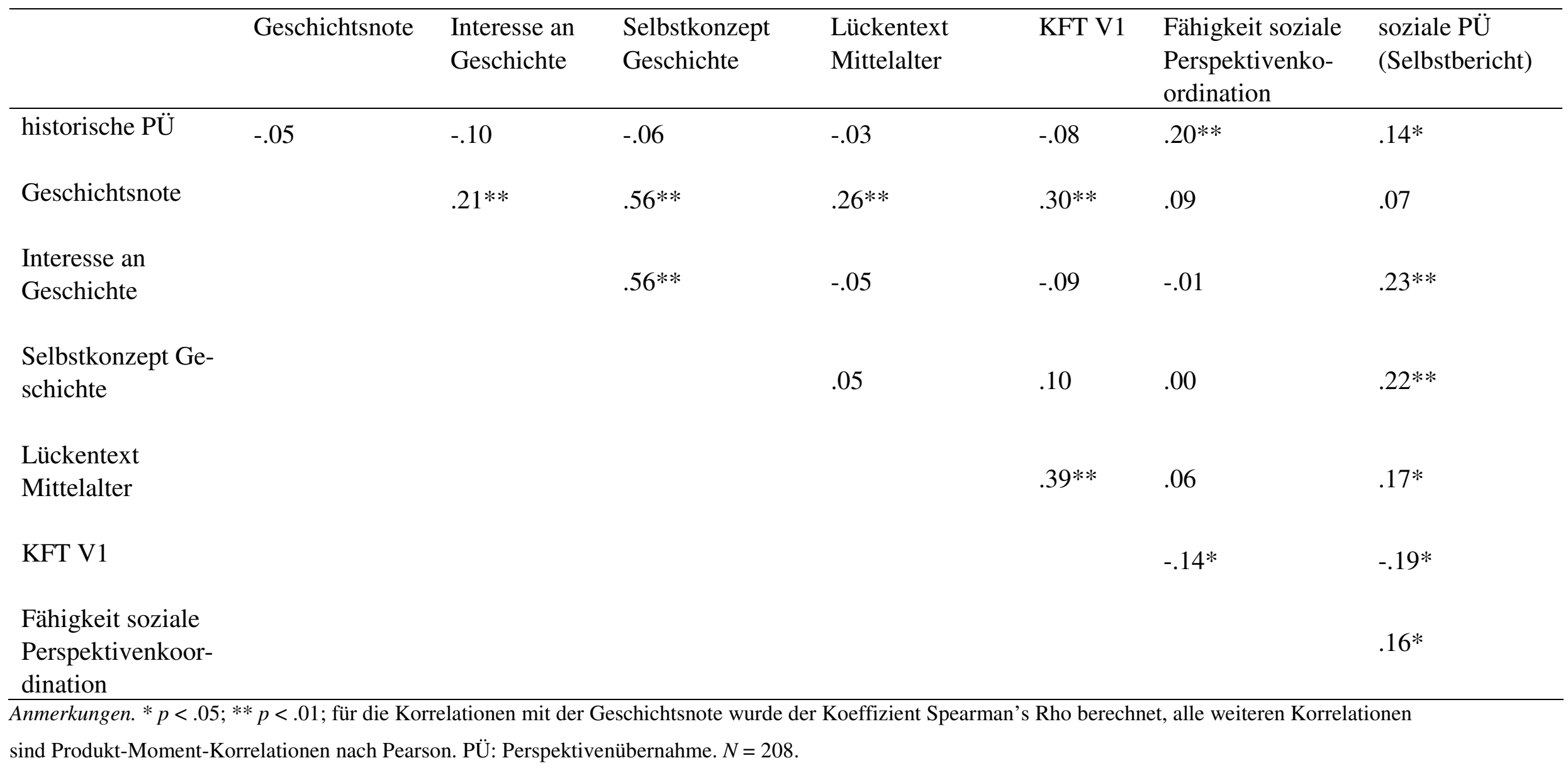


Tabelle 11: Korrelationen, Klassenstufe 10

\begin{tabular}{|c|c|c|c|c|c|c|c|}
\hline & Geschichtsnote & $\begin{array}{l}\text { Interesse an } \\
\text { Geschichte }\end{array}$ & $\begin{array}{l}\text { Selbstkonzept } \\
\text { Geschichte }\end{array}$ & $\begin{array}{l}\text { Lückentext } \\
\text { WR/ 2. WK }\end{array}$ & KFT V1 & $\begin{array}{l}\text { Fähigkeit soziale } \\
\text { Perspektivenkoor- } \\
\text { dination }\end{array}$ & $\begin{array}{l}\text { soziale PÜ } \\
\text { (Selbstbericht) }\end{array}$ \\
\hline historische PÜ & $.39 * *$ & $.44 * *$ & $.48 * *$ & $.25 * *$ & .13 & -.05 & -.12 \\
\hline Geschichtsnote & & $.50 * *$ & $.65 * *$ & $.21 * *$ & $.26 * *$ & .00 & .00 \\
\hline $\begin{array}{l}\text { Interesse an } \\
\text { Geschichte }\end{array}$ & & & $.65 * *$ & $.39 * *$ & $.25 * *$ & -.14 & .04 \\
\hline $\begin{array}{l}\text { Selbstkonzept } \\
\text { Geschichte }\end{array}$ & & & & $.37 * *$ & $.21 * *$ & -.15 & -.04 \\
\hline $\begin{array}{l}\text { Lückentext } \\
\text { WR/ 2. WK }\end{array}$ & & & & & $.48 * *$ & -.02 & .05 \\
\hline KFT V1 & & & & & & .02 & .06 \\
\hline $\begin{array}{l}\text { Fähigkeit soziale } \\
\text { Perspektivenkoor- } \\
\text { dination }\end{array}$ & & & & & & & .11 \\
\hline
\end{tabular}


Tabelle 11 zeigt die Korrelationstabelle für die 10. Klassenstufe. Auch in dieser Altersgruppe hängen die fachspezifischen Leistungs- und Motivationsindikatoren positiv miteinander zusammen. Interesse und Geschichtsnote korrelieren $\mathrm{zu} r=.50$. Das Selbstkonzept korreliert zu $r=.65$ sowohl mit der Geschichtsnote als auch mit dem Interesse an Geschichte. Die Kompetenz zur historischen Perspektivenübernahme korreliert signifikant mit allen fachspezifischen Motivations- und Leistungsvariablen, am stärksten mit dem Interesse $(r=.44)$ und dem Selbstkonzept in Geschichte $(r=.48)$. Auch zum Abschneiden im Lückentext $(r=.25)$ und zur Geschichtsnote $(r=.39)$ bestehen signifikante Zusammenhänge. Die Kompetenz zur historischen Perspektivenübernahme hängt in dieser Klassenstufe nicht mit den beiden Indikatoren sozialer Perspektivenübernahme zusammen.

\subsection{Zusammenfassung der Ergebnisse}

Ziel dieser explorativen Studie war es, einen ersten Einblick in das Zusammenspiel zwischen einer Teilkompetenz historischen Denkens (historische Perspektivenübernahme), fachspezifischen Motivations- und Leistungsindikatoren und der sozialkognitiven Fähigkeit zur sozialen Perspektivenübernahme zu erhalten.

Unsere erste Vermutung war, dass sich auch im Fach Geschichte positive $\mathrm{Zu}$ sammenhänge zwischen der Schulnote, dem fachspezifischen Interesse und dem Selbstkonzept zeigen. Diese Vermutung konnte für beide untersuchten Klassenstufen bestätigt werden. Dieser Befund steht im Einklang mit den Ergebnissen von Rost und Sparfeldt (2002). Dass die Stärke der Zusammenhänge in der 10. Klasse durchweg höher ist als in der 7. Klasse, ist außerdem konsistent mit den Daten für die Fächer Englisch, Naturwissenschaften und Mathematik aus der Längsschnittstudie von Denissen et al. (2007). Vor allem der Zusammenhang zwischen der Geschichtsnote und dem Interesse an Geschichte ist in der 10. Klasse deutlich höher als in der 7. Klasse (.50 vs .21).

Zweitens haben wir untersucht, welche Zusammenhänge zwischen einer Teilkompetenz historischen Denkens - der historischen Perspektivenübernahme - und den fachspezifischen Leistungs- und Motivationsindikatoren bestehen. Während wir in der 7. Klasse keinerlei substanzielle Zusammenhänge feststellen konnten, bestehen in der 10. Klasse hochsignifikante Korrelationen der historischen Perspektivenübernahme mit allen fachspezifischen Merkmalen. Insbesondere das Interesse und das Selbstkonzept 
korrelieren relativ hoch (.44 und .48) mit der kompetenten Bearbeitung des historischen Szenarios, aber auch die Geschichtsnote (.39) und die Beantwortung des themenspezifischen Lückentexts (.25) hängen statistisch bedeutsam mit der Kompetenz zur historischen Perspektivenübernahme zusammen.

Drittens sind wir der Frage nachgegangen, ob Zusammenhänge zwischen der Kompetenz zur historischen Perspektivenübernahme und zwei Indikatoren für das sozial-kognitive Merkmal der sozialen Perspektivenübernahme bestehen. Zum einen wurde die Fähigkeit zur sozialen Perspektivenübernahme mit offenem Antwortformat in Anlehnung an Selman (1980) und Gutzwiller-Helfenfinger (2003) erhoben. Zum anderen wurde die Bereitschaft, Perspektiven von Interaktionspartnern im Alltag zu übernehmen, mit einem Selbstberichtsverfahren erfasst. Während in der 10. Klasse keinerlei Zusammenhänge zwischen den fachspezifischen und den sozial-kognitiven Merkmalen bestehen, deuten die Befunde der 7. Klasse auf eine Verbindung zwischen historischer und sozialer Perspektivenübernahme hin. Die Korrelationen liegen im niedrigen bis moderaten Bereich - ein Befund, der sich mit den Ergebnissen Gehlbachs (2004a) deckt. Die Bereitschaft, Perspektiven anderer Personen im Alltag einzunehmen, hängt in der 7. Klasse außerdem mit dem Interesse und Selbstkonzept im Fach Geschichte zusammen.

Da diese pädagogisch und entwicklungspsychologisch interessanten Ergebnisse innerhalb einer explorativen Querschnittsstudie zustande gekommen sind und auf korrelativen Daten basieren, sind daraus nur begrenzt Schlussfolgerungen über die Entwicklung bzw. den Erwerb der Kompetenz zur historischen Perspektivenübernahme möglich. Um unsere Ergebnisse für Theoriebildung und weitere empirische Untersuchungen nutzbar zu machen, gehen wir im folgenden Abschnitt auf mögliche Wirkzusammenhänge ein, im Bewusstsein, dass diese Vermutungen aufgrund der vielfältigen Grenzen der Studie zum jetzigen Zeitpunkt noch sehr spekulativ bleiben müssen.

Zuvor möchten wir noch einen Randbefund dieser Studie erwähnen, der auf den ersten Blick verwunderlich erscheint. Die Fähigkeit zur sozialen Perspektivenübernahme korreliert in unseren Daten nur gering (in der 7. Klasse) bis gar nicht (in der 10. Klasse) mit der Bereitschaft zur Perspektivenübernahme. Dies kann mehrere Gründe haben. Zum einen muss auf einen möglichen Methodeneffekt aufgrund der unterschiedlichen Erhebungsmethoden hingewiesen werden - offenes vs. geschlossenes Antwortformat. Zum anderen weist Steins (1998) darauf hin, dass Selbstberichtsverfahren zu 
Perspektivenübernahme und Empathie einer starken Tendenz zur sozialen Erwünschtheit unterliegen können.

\subsection{Mögliche Wirkzusammenhänge sozial-kognitiver und geschichtsspezifischer Schülermerkmale}

Unsere Studie wirft zahlreiche Fragen auf, die das Wirkungsgefüge zwischen fachspezifischen und sozial-kognitiven Schülermerkmalen bezogen auf das Fach Geschichte betreffen. Ausgehend von den Daten der 7. Klasse sollte zukünftig untersucht werden, ob die Kompetenz zur historischen Perspektivenübernahme bei Schülern zunächst auf deren sozial-kognitive Fähigkeit und Bereitschaft zurückzuführen ist, im Alltag fremde Perspektiven einzunehmen. Naheliegend ist außerdem die Frage, ob eine solche sozial-kognitive Kompetenz das Interesse an Fächern wie Geschichte fördern kann. Die von uns gefundene Korrelation zwischen der Bereitschaft zur Perspektivenübernahme und dem Geschichtsinteresse stellt dafür eine erste notwendige, wenngleich noch keinesfalls hinreichende Bedingung dar.

Die Daten der 10. Klasse regen zu weiteren Fragen und Vermutungen an: Historische Perspektivenübernahme reiht sich in dieser Klassenstufe in das Beziehungsgefüge der fachspezifischen Leistungs- und Motivationsindikatoren ein. Die Fähigkeit und Bereitschaft zur sozialen Perspektivenübernahme hängt mit diesen fachspezifischen Merkmalen nicht mehr zusammen. Dieser Befund sollte in zukünftigen Studien überprüft werden und mit spezifischen Merkmalen des Unterrichts in Verbindung gebracht werden. Es scheint zwar im Zuge einer höheren fachlichen Spezialisierung plausibel, aber aus fachdidaktischer Sicht wenig wünschenswert, dass allgemeine sozial-kognitive Fähigkeiten im Fach Geschichte in höheren Jahrgängen eine geringere Rolle spielen vor allem da Studien wie die von Hickey Schultz et al. (2001) auf die Potentiale des Faches Geschichte für den Lebensweltbezug der Schüler hinweisen.

Bezogen auf die Unterschiede zwischen den beiden Klassenstufen lassen sich entwicklungspsychologisch relevante Fragen anschließen: Stellt die sozial-kognitive Fähigkeit zur sozialen Perspektivenübernahme bei Kindern eine Voraussetzung für das Sich-Hineinversetzen in historische Situationen dar? Führt eine egozentrische Sichtweise dazu, dass historische Situationen ausschließlich nach den eigenen Wertmaßstäben der Gegenwart beurteilt werden können? Und welche Prozesse sind für eine mögliche 
Entwicklung dieser Kompetenzen verantwortlich? Wie sollte Geschichtsunterricht gestaltet werden, um eine aufeinander abgestimmte Förderung sozialer und fachspezifischer Kompetenzen zu ermöglichen?

Diese Fragen und Vermutungen, die sich aufgrund der empirischen Befunde ergeben, können zur weiteren Auseinandersetzung mit dem komplexen Wirkungsgefüge im Fach Geschichte anregen, müssen aber noch als sehr vorläufig betrachtet werden. Das ist zum einen im spezifischen Aufbau unserer explorativen Querschnittsstudie begründet. Zum anderen weisen die Grenzen unserer Studie aber auch auf allgemeine Herausforderungen für die empirische Kompetenzforschung in bisher wenig untersuchten Schulfächern und an der Schnittstelle zwischen Erziehungswissenschaft, Fachdidaktik und Psychologie hin.

\subsection{Grenzen der Studie}

\subsubsection{Validität des Konstruktes}

Historische Perspektivenübernahme wird in der geschichtsdidaktischen Diskussion sehr breit gefasst. Schon durch die vielfältigen Begrifflichkeiten - z.B. historical empathy bei Ashby und Lee (1987); Perspektivenübernahme oder Wahrnehmung von Perspektivität bei Sauer (2006); Welt- und Fremdverstehen bei Körber, Schreiber et al. (2007) - wird deutlich, dass das Konstrukt noch nicht sehr eng umrissen ist. Auch bezüglich der Bedeutung von historischem Wissen für diese Kompetenz gibt es noch keine einheitliche Auffassung, auch wenn Davis Jr. (2001) sowie Ashby und Lee (1987) die Relevanz von Kontextwissen für historische Perspektivenübernahme betonen. In unserer Studie haben wir versucht, einen ersten empirischen Zugang mithilfe eines historischen Kontextes zu schaffen, zu dem Schüler bereits durch den Unterricht vermitteltes Wissen haben sollten. Unsere Befunde sprechen dafür, dass historische Perspektivenübernahme mehr ist als reines historisches Kontextwissen. Zwar gibt es in Klasse 10 eine signifikante Korrelation mit der Kenntnis zentraler Fakten zu dem Themengebiet des Szenarios; die Größenordnung von $r=.25$ lässt jedoch die Interpretation zu, dass historische Perspektivenübernahme nicht ausschließlich vom historischen Wissen der Schüler gespeist wird. 
Bei der Messung der Kompetenz haben wir uns in dieser Studie auf zwei Aspekte beschränkt, die von mehreren Autoren (z.B. Ashby \& Lee, 1987; Bergmann, 2000; von Borries, 1995; Sauer, 2006; Seixas, 1996) als zentral für historische Perspektivenübernahme angesehen werden - die Gegenwartsfixierung, die überwunden werden soll, und die Berücksichtigung des historischen Kontextes beim Verstehen der Akteure, die als Ziel der Entwicklung dieser Kompetenz gilt. Offen bleibt, ob es weitere zentrale Bestandteile von historischer Perspektivenübernahme gibt, die mit unserem Instrument nicht erfasst wurden. Es sind weitere Explikationen des Konstruktes notwendig, die sich unseres Erachtens sowohl an fachspezifischen als auch an pädagogisch-psychologischen Theorien zur Perspektivenübernahme orientieren sollten. Auch weitere qualitative Studien im Sinne von Ashby und Lee (1987) können dazu beitragen, Bestandteile historischer Perspektivenübernahme im Denken von Schülern aufzudecken.

Darüber hinaus ist im Hinblick auf die Validität diskussionswürdig, ob die für die soziale Perspektivenübernahme vorgeschlagene Unterteilung zwischen Fähigkeit und Bereitschaft (Gehlbach, 2004b) auch für das Konzept der historischen Perspektivenübernahme nützlich sein kann. In Weinerts Konzeption (2001) von Kompetenz fließen sowohl Fähigkeiten als auch Bereitschaften ein, bei der konkreten Umsetzung in Kompetenzaufgaben kann man diesem Anspruch jedoch nur schwer gerecht werden. Letztlich muss jedes Testergebnis als ein Ausdruck des Zusammenspiels von Fähigkeiten und Bereitschaften angesehen werden.

\subsubsection{Aufgabenformate}

In der vorliegenden Studie wurde historische Perspektivenübernahme bei Schülern über die Präsentation eines Szenarios und die anschließende Beantwortung von Items mithilfe von Ratingskalen gemessen. Dies stellt insofern eine Ausnahme dar, als die meisten geschichtsdidaktischen Studien qualitative Studiendesigns unter Verwendung offener Antwortformate nutzen (z.B. Ashby \& Lee, 1987; Shemilt, 1987; Voss \& Wiley, 1997; Wineburg, 1991). Um Erkenntnisse über die Prozesse historischen Denkens zu sammeln, scheint dies auch der geeignete Weg zu sein. Insbesondere im Kontext von Bildungsstandards ist es jedoch notwendig, auch die Chancen und Grenzen quantitativer Studien auszuloten, da eine ökonomische Messung von Kompetenzen in größeren Schülerpopulationen erst dadurch möglich wird. Über spezifische Messprob- 
leme, die mit der Natur historischer Denkprozesse in Verbindung stehen, sind weitere Diskussionen notwendig. Beispielsweise verweist von Borries (2007) darauf, dass es im Bereich des historischen Denkens oftmals nicht die eine richtige Antwort geben kann. Vielmehr kommt es auf historisch plausible Argumentationen und Begründungen an, die Schüler entwickeln sollen und die zu durchaus unterschiedlichen Interpretationen historischer Ereignisse führen könnten. Unserer Ansicht nach kann die Verwendung von Ratingskalen einen Kompromiss darstellen, da sie nicht eine einzig richtige Antwort impliziert, sondern jedes Item abgestuft und unabhängig von den anderen Items bewertet werden kann. Somit bleibt Schülern die Möglichkeit, vermeintlich gegensätzliche Aussagen in derselben Art und Weise zu bewerten, falls dies ihren historischen Konzepten entsprechen sollte. Auch wenn dies ein Problem für eine einfache Skalenbildung darstellt, eröffnet es Wissenschaftlern doch die Chance, Widersprüche und Misskonzepte $\mathrm{zu}$ erkennen und neue Erkenntnisse zu gewinnen. Bezogen auf die Prozessanalyse historischen Denkens kann jedoch auch die Verwendung von Ratingskalen offene Antwortformate und Methoden des lauten Denkens (Wineburg, 1991) nicht ersetzen.

$\mathrm{Zu}$ diskutieren ist weiterhin die Verwendung fiktiver Szenarien, da es im Schulfach Geschichte darum geht, das Verständnis realer historischer Begebenheiten bei Schülern zu fördern. Solche eigens für den Zweck der Studie konstruierten Aufgaben haben den Vorteil, dass weniger Störfaktoren in die Messung hineinspielen, die bei der Verwendung authentischer Quellen und Darstellungen wirksam sind. Dies betrifft beispielsweise die sprachliche Komplexität der untersuchten Dokumente und die Vermischung von verschiedenen Teilprozessen historischen Denkens (z.B. das Ineinandergreifen von Quelleninterpretation und historischer Perspektivenübernahme). Dass man mit der Verwendung fiktiver Szenarien jedoch unter Umständen Einbußen in Bezug auf die Inhaltsvalidität in Kauf nimmt, soll an dieser Stelle nicht unerwähnt bleiben.

\subsubsection{Reliabilität}

Weiterhin muss auf die zum Teil geringen internen Konsistenzen vor allem bei der Erfassung historischer Perspektivenübernahme in der 7. Klasse eingegangen werden. Vermutlich setzt es bereits ein gewisses $\mathrm{Ma} ß$ an historischer Perspektivenübernahme voraus zu erkennen, ob die entsprechenden Aussagen gegenwartsfixiert sind oder den historischen Kontext einbeziehen. Dies gelingt in Klasse 7 vermutlich weniger 
gut als in Klasse 10, was zu einer geringeren internen Konsistenz der Skala in dieser Altersgruppe führen könnte. Um dieses Problem zu lösen, müssten die Items für Klasse 7 überarbeitet werden. Wir vermuten allerdings, dass die Zusammenhänge zu den fachspezifischen Variablen sich auch bei einer Erhöhung der Reliabilität nicht grundlegend verändern würden - die Korrelationen mit den fachspezifischen Variablen, die durchgehend um Null liegen, legen diese Interpretation nah.

$\mathrm{Zu}$ diskutieren bleibt, ob der Maßstab einer hohen internen Konsistenz, wie er in der Psychologie in der Regel angelegt wird, für die Erforschung historischen Lernens ebenso rigoros vorauszusetzen ist. Es ist fraglich, ob es der Breite der Konstrukte, die den geschichtsdidaktischen Diskurs bestimmen, gerecht wird, wenn ausschließlich die interne Konsistenz von Skalen berücksichtigt wird. Insbesondere bei den ersten Studien in diesem Bereich, die sich auf die zum Teil noch recht vage formulierten Kompetenzmodelle stützen und sich an heterogenen Kompetenzbereichen orientieren müssen, sollten geringere interne Konsistenzen zwar kritisch reflektiert werden, jedoch nicht automatisch zur Eindämmung der Forschungsaktivitäten im Fach führen. Denn auch mit heterogenen Konstrukten und ersten empirischen Ansätzen ist eine Annäherung an interessante inhaltliche Fragestellungen möglich.

\subsubsection{Kontextgebundenheit}

Durch die Anlage der Studie, die historische Perspektivenübernahme in den Klassen 7 und 10 an zwei unterschiedlichen historischen Kontexten erfasst, muss der Mangel an Vergleichbarkeit der Aufgaben über die Klassenstufen hinweg diskutiert werden. Auch wenn bei der Konstruktion der Texte darauf geachtet wurde, Charakteristika wie Textlänge, Schwierigkeit und Formulierung der Items für beide Klassenstufen möglichst einheitlich zu gestalten, bleiben die historischen Kontexte unterschiedlich. Wir haben uns in dieser Studie dafür entschieden, unterschiedliche Kontexte für die beiden Klassenstufen zu wählen, damit die Schüler ihre Kompetenz zur historischen Perspektivenübernahme an zuvor im Unterricht behandelten historischen Themen zeigen konnten. Wir vermuten, dass historische Kontextualisierung (unser Anspruch an kompetente Schüler) ein Mindestmaß an Kenntnissen über den spezifischen historischen Kontext voraussetzt (z.B. Ashby \& Lee, 2001; Davis Jr., 2001). Es wäre demnach nicht möglich, historische Perspektivenübernahme kontextunabhängig oder an gänzlich un- 
bekannten Kontexten zu erfassen. Die Befunde von Beck und McKeown (1994) lassen es zudem zweifelhaft erscheinen, ob sich Zehntklässler noch an Inhalte aus dem Geschichtsunterricht der siebten Klasse erinnern. Andererseits sollen Kompetenzen im Sinne der Expertise von Klieme et al. (2003) aber nicht nur an einem spezifischen Kontext bei Schülern nachgewiesen werden, sondern sich an variablen Kontexten manifestieren und sich als transferfähig herausstellen. Diese Herausforderungen in Bezug auf die Kontextgebundenheit historischer Kompetenzen sind weiter zu diskutieren, will man Kompetenzmessung im Fach Geschichte an Schülern unterschiedlicher Altersstufen mit unterschiedlichen Unterrichtsinhalten betreiben.

\subsubsection{Kompetenzstufen historischen Denkens?}

Mit der vorliegenden Studie haben wir das Konzept historische Perspektivenübernahme durch zwei Aspekte definiert, die in der Literatur weitgehend übereinstimmend als wichtig angesehen werden (z.B. Ashby \& Lee, 1987; Bergmann, 2000; von Borries, 1995; Sauer, 2006; Seixas, 1996). Dies lässt viele theoretische und methodische Fragen offen, beispielsweise ob Schüler immer mit einer gegenwartsfixierten Betrachtung historischer Kontexte beginnen, ob mit historischer Kontextualisierung, so wie sie von uns operationalisiert wurde, bereits die höchste Kompetenzausprägung erreicht ist, und auf welchen Wegen Schüler von Gegenwartsfixierung zu einer kontextualisierten Betrachtung historischer Situationen gelangen. Diese Fragen berühren die Forderung nach der Stufung von Kompetenzen (Klieme et al., 2003). Unser Beitrag liefert ausschließlich einen Vorschlag für zwei „Endpunkte“ der Kompetenz zur historischen Perspektivenübernahme. Sowohl für die Unterteilung quantitativ definierbarer Kompetenzstufen (in Form von erreichten Punktzahlen in Lernstandserhebungen) als auch für die Ableitung qualitativer Entwicklungsstufen (z.B. mit der Möglichkeit von Entwicklungssprüngen und der Aufhebung von Fehlkonzepten) reichen die bisher gesammelten Erkenntnisse in diesem Feld in keiner Weise aus. Dies gilt nicht nur für die Kompetenz, historische Perspektiven einzunehmen, sondern vermutlich für die meisten Teilbereiche historischen Denkens.

Neben all diesen Herausforderungen, mit denen empirisch arbeitende Forscher im Fach Geschichte konfrontiert sind, sollten die damit verbundenen Chancen nicht 
unterschätzt werden. Denn erst durch die Sammlung empirischer Daten und die Formulierung exakterer Hypothesen können wir erkennen, welche fachspezifischen und sozialen Kompetenzen durch die Beschäftigung mit der Vergangenheit bei Schülern gefördert werden können. Dies kann letztlich zur besseren gesellschaftlichen Legitimation des Unterrichtsfaches Geschichte beitragen. 


\section{Perspektivenübernahme als sozial-kognitive Kompetenz historischen Verstehens - Diskussion und Ausblick}

\subsection{Theoretische und empirische Erträge}

In einem bisher nur wenig beachteten Forschungsfeld ist es vergleichsweise leicht zu neuen Erkenntnissen zu gelangen. Andererseits bedürfen empirische Befunde, um als gesichert gelten zu können, der Replikation durch unabhängige Studien. Beides gleichzeitig anzustreben - neue Erkenntnisse sowie gesicherte Aussagen - erscheint höchst anspruchsvoll und nur mit immensen zeitlichen und finanziellen Ressourcen realisierbar. Am Ende dieser Arbeit müssen wir uns also mit vorläufigen Erkenntnissen zufriedengeben, für die noch zahlreiche empirische Bewährungsproben ausstehen.

Der theoretische Ertrag dieser Arbeit besteht darin, das Konzept der Perspektivenübernahme aus zwei Blickwinkeln - dem geschichtsdidaktischen und dem sozialkognitiven entwicklungspsychologischen - zu beleuchten und Anschlussmöglichkeiten aufzuzeigen. Am Ende dieser interdisziplinären Auseinandersetzung steht folgende Arbeitsdefinition von Perspektivenübernahme als Kompetenz historischen Verstehens:

Historische Perspektivenübernahme ist eine sozial-kognitive Kompetenz, sich in Akteure aus der Vergangenheit hineinzuversetzen und dabei über die eigene gegenwärtige Sichtweise hinaus historische Denk- und Handlungshorizonte auf Basis der damals geltenden Bedingungen zu rekonstruieren.

Die empirischen Erträge lassen sich weniger leicht komprimieren. In Anlehnung an die drei Publikationen lassen sich jedoch zwei Bereiche identifizieren, in denen neue Erkenntnisse gewonnen werden konnten: Die Entwicklung eines Messzugangs und die Betrachtung von Zusammenhängen zwischen fachspezifischen und sozial-kognitiven Merkmalen.

Historische Perspektivenübernahme einer empirisch-quantitativen Erfassung zugänglich zu machen, ist in dieser Arbeit weitgehend geglückt. Durch den Einsatz historisch plausibler fiktiver Szenarien und eines Ratingskalen-Formats können größere Schülerstichproben bezogen auf ihre Kompetenz zur historischen Perspektivenübernahme untersucht werden. Für das Szenario der 10. Klasse (historischer Kontext: Wahlentscheidung in der Weimarer Republik) werden die elementaren psychometrischen 
Anforderungen der Reliabilität und Kriteriumsvalidität erfüllt. Schüler können zuverlässig drei Typen historischer Perspektivenübernahme zugeordnet werden und diese Klassifikation hängt mit der Schulnote im Fach Geschichte zusammen. Für das Szenario der 7. Klasse (historischer Kontext: Entscheidung von Eltern, ihre Tochter in ein Kloster zu geben zur Zeit des Mittelalters) fällt das abschließende Urteil verhaltener aus. Die Klassifikation von Schülern anhand ihrer Kompetenz zur historischen Perspektivenübernahme ist zwar ebenfalls möglich, die gefundenen Typen können jedoch nicht eindeutig im Sinne der theoretischen Annahmen in Bezug auf höhere bzw. geringere Kompetenz interpretiert werden. Dies erschwert auch die Überprüfung von Validitätsaspekten.

Bevor dieser erste empirische Zugang im Rahmen der Umsetzung von Bildungsstandards Anwendung im Schulkontext finden kann, sind für beide Szenarien Modifikationen notwendig um die Kompetenz historischer Perspektivenübernahme angemessen empirisch abzubilden. Dies betrifft insbesondere den theoretischen Aspekt der Rolle des historischen Akteurs, der sich in den durchgeführten Analysen nicht als der theoretisch plausible Zwischenschritt zwischen Gegenwartsfixierung und historischer Kontextualisierung herauskristallisierte. $\mathrm{Ob}$ dies auf falsche theoretische Überlegungen oder auf Probleme bei der empirischen Umsetzung zurückzuführen ist, bleibt nach Abschluss dieser Arbeit noch ungeklärt - wahrscheinlich ist es eine Kombination aus beidem.

Abschließend lässt sich für die Entwicklung des Messzugangs zur Erfassung historischer Perspektivenübernahme ein positives Fazit ziehen. Die vorliegenden empirischen Befunde erweitern das Verständnis von Perspektivenübernahme als historische Kompetenz, die bisher fast ausschließlich in normativ angelegten Kompetenzmodellen berücksichtigt wurde. Es existiert nun eine erste empirisch gestützte Einschätzung, dass die Berücksichtigung des historischen Kontextes bei ca. 90\% der Zehntklässler mehr oder weniger gelingt. Auch innerhalb dieser großen Gruppe können jedoch noch Kompetenzunterschiede zwischen Schülern festgestellt werden, die Bezüge zur Geschichtsnote aufweisen. Für die 7. Klasse lässt sich vorläufig konstatieren, dass Gegenwartsfixierung bei annähernd allen Schülern vorherrschend ist und dass die Berücksichtigung des historischen Kontextes allenfalls in ersten Ansätzen gelingt.

Die vorliegende Arbeit liefert weiterhin erste empirische Erkenntnisse über Perspektivenübernahme im Zusammenspiel mit fachspezifischen und sozial-kognitiven Schülermerkmalen. Die in Kapitel 5 dargestellten Befunde weisen auf einen Zusammenhang zwischen sozialer und historischer Perspektivenübernahme in Klasse 7 hin, während historische Perspektivenübernahme in Klasse 10 gänzlich in das Gefüge aus 
fachspezifischer Leistung, Selbstkonzept und Interesse eingebettet ist. Trotz methodischer Mängel der Studie lassen diese Befunde erste Spekulationen über das Wirkungsgefüge $\mathrm{zu}$, in dem sich die Kompetenz zur historischen Perspektivenübernahme im Kontext Schule entwickelt, und bieten somit Anknüpfungspunkte für weitere empirische Arbeiten zur Erforschung von Kompetenzen historischen Verstehens.

\subsection{Implikationen für die Messung historischer Kompetenzen}

Neben der Diskussion der spezifischen empirischen Befunde zur Kompetenz historischer Perspektivenübernahme bei Schülern der Klassenstufen 7 und 10 gibt diese Arbeit Anlass zur Reflexion über die Erfassung von Kompetenzen historischen Verstehens im Kontext der Debatte um Bildungsstandards für das Schulfach Geschichte.

Die Formulierung und Messung historischer Kompetenzen stellt Wissenschaftler, bildungspolitische Entscheidungsträger sowie Lehrende vor immense Herausforderungen. Sie beginnen bei der Einigung auf Bildungsziele auf Ebene des Schulsystems also zentrale Erwartungen an Fähigkeiten, Fertigkeiten, Werthaltungen und Einstellungen, die Schüler durch den Unterricht in den verschiedenen Fächern erwerben sollen (Klieme et al., 2003). Sie setzen sich fort bei der Formulierung fachspezifischer Kompetenzmodelle, die für alle Schulformen und Klassenstufen Gültigkeit haben sollen. Und diese Herausforderungen kumulieren, wenn Kompetenzen durch Messinstrumente abgebildet werden sollen, sodass die Formulierung von Mindeststandards für Schüler aller Altersstufen möglich wird.

Aufgrund einer einzelnen empirischen Arbeit wie der vorliegenden kann man für diese Herausforderungen keine tragfähigen Lösungen entwickeln. Auf Basis der Erfahrungen aus dieser Dissertation können jedoch einzelne Aspekte herausgegriffen werden, die für die Formulierung und Erfassung von Kompetenzen relevant erscheinen. Zwei dieser Aspekte werden nachfolgend thematisiert, da sie für die vorliegende Arbeit im Kontext des Graduiertenkollegs „Passungsverhältnisse schulischen Lernens“ handlungsleitend waren. Der erste betrifft die Zusammenarbeit verschiedener Fachdisziplinen, der zweite den Anspruch der Formulierung von „Kompetenzstufen historischen Verstehens“", wie ihn der Name des Projekts nahelegt. 


\subsubsection{Interdisziplinarität als Herausforderung und Chance}

Für die Konzeption und Erfassung von Perspektivenübernahme als Kompetenz historischen Verstehens wurde in dieser Arbeit sowohl auf Ansätze der Geschichtsdidaktik als auch auf Theorien und Methoden aus der Psychologie zurückgegriffen. Eine solche interdisziplinäre Betrachtung erweist sich in vielerlei Hinsicht als gewinnbringend: Die Fachdidaktik liefert Hinweise darauf, wie sich Perspektivenübernahme im Kontext Geschichte äußert, welche Charakteristika sie aufweist und warum sie für das historische Verstehen unerlässlich ist (z.B. Ashby \& Lee, 1987; Körber, Schreiber et al., 2007; Sauer, 2006). Die Psychologie mit ihrer deutlich längeren empirischen Forschungstradition liefert Einblicke in weitgehend universell gültige Entwicklungsmuster der Fähigkeit zur sozialen Perspektivenkoordination (Selman, 1980). Diese können um Befunde ergänzt werden, die spezifische Kontextfaktoren in den Blick nehmen und Anschlussmöglichkeiten für die Erforschung von Perspektivenübernahme im Fach Geschichte bieten (z.B. Feigenberg et al., 2008; Gehlbach, 2004a; 2004b; Wainryb, 1993). Ein Vorgehen, welches diese beiden Forschungsstränge verknüpft, eröffnet zahlreiche Chancen, die ohne die Berücksichtigung der jeweils anderen Disziplin verborgen bleiben würden, sowohl in Bezug auf die theoretische Konzeption als auch auf die methodische Umsetzung. Doch wie bereits in der Einleitung dieser Arbeit angeklungen, bringt die Kompetenzforschung im Fach Geschichte diverse Herausforderungen mit sich, und diese äußern sich auch (und vielleicht insbesondere) im Spannungsfeld interdisziplinären Arbeitens.

Eine erste Herausforderung betrifft die interdisziplinäre Theoriebildung. Wie in Kapitel 1 und 2 ersichtlich, findet die Auseinandersetzung über Perspektivenübernahme in Geschichtsdidaktik und Psychologie auf sehr unterschiedliche Art und Weise statt. Während Perspektivenübernahme als historische Kompetenz in den Prozess historischer Erkenntnisgewinnung eingebettet ist (Yeager \& Foster, 2001) und sich in vielen Kompetenzmodellen nicht als isolierbare Kompetenz verorten lässt, steht Perspektivenübernahme in der Psychologie als sozial-kognitive Fähigkeit als ein relativ klar umrissenes Konstrukt da und lässt sich beispielsweise von dem der Empathie unterscheiden. Um Ideen aus beiden Fachdisziplinen zu nutzen, ist es essentiell, die gemeinsamen theoretischen Vorstellungen hinter den unterschiedlichen Begrifflichkeiten und inhaltlichen Akzentuierungen sichtbar zu machen. Darüber hinaus wurde an diversen Stellen im Prozess dieser Arbeit deutlich, dass Interdisziplinarität am besten dann gelingt, wenn 
beide Fachkulturen ihre spezifischen theoretischen Vorstellungen einbringen können und nicht der einen Disziplin Theorien der anderen Disziplin „übergestülpt“ werden. Diese Gefahr besteht sicherlich vornehmlich aus Sicht der Geschichtsdidaktik, da psychologische Modelle wie auch das zur sozialen Perspektivenkoordination (Selman, 1980) zumindest in ihrer ursprünglichen Form prinzipiell universelle Gültigkeit beanspruchen. Dass eine Debatte über die Gemeinsamkeiten und Besonderheiten des historischen und des sozialen Verstehens jedoch für beide Fachdisziplinen anregend ist, zeigt sich erst durch die interdisziplinäre Kooperation (z.B. Sauer, Martens, Hartmann \& Hasselhorn, in Vorbereitung; Stearns, Seixas \& Wineburg, 2000; Voss \& Carretero, 1998).

Eine zweite Herausforderung betrifft die methodische Herangehensweise der beiden Disziplinen. Der Anspruch der Geschichtswissenschaft an eine möglichst umfassende Rekonstruktion von historischen Prozessen und Ereignissen steht in vielerlei Hinsicht im Kontrast zu der Arbeitsweise einer quantitativ-empirisch forschenden Psychologie. Zwei Beispiele aus der vorliegenden Arbeit illustrieren die daraus entstehenden Schwierigkeiten: Um einen Kontext zu schaffen, in den sich Schüler hineinversetzen können, wurde mit fiktiven Szenarien gearbeitet, die historisch plausible Situationen abbilden. Dies ist für die psychologische Forschung zur Perspektivenübernahme nichts Ungewöhnliches (z.B. Gutzwiller-Helfenfinger, 2003; Mischo, 2005; Selman, 1980) und die Vorteile liegen auf der Hand: Den Schülern wird ein gut verständlicher Text präsentiert; untersucht man verschiedene Klassenstufen, kann man die Länge der Texte vergleichbar gestalten; alle Informationen, die notwendig sind, um die anschließenden Fragen verstehen zu können, werden bereitgestellt, und je nach Forschungsfrage lassen sich verschiedene Aspekte des Szenarios variieren. Für die Geschichtswissenschaft ist ein solches Vorgehen jedoch weit weniger üblich und birgt spezielle Probleme: Das Szenario stellt keinen Ausschnitt der historischen Realität dar, sondern ist bereits aus gegenwärtiger Perspektive konstruiert. Merkmale wie eine altertümliche Sprache fehlen und die für die Fachwissenschaft und -didaktik zentrale Unterscheidung zwischen Quelle und Darstellung erscheint bei einem fiktiven Szenario nicht sinnvoll. Historische Perspektivenübernahme wird also nicht als Teil eines realen historischen Erkenntnisprozesses untersucht, sondern isoliert als einzelne Kompetenz betrachtet. Damit stellt sich die Frage, ob noch von historischer Perspektivenübernahme im Sinne der in Kapitel 1 vorgestellten Kompetenzmodelle die Rede ist. Ein weiteres Beispiel betrifft die Formulierung der Items, die die verschiedenen Aspekte historischer Perspektivenübernahme ab- 
bilden sollen. Aus quantitativ-psychologischer Sicht stellt auch dieses Vorgehen kein schwerwiegendes Problem dar. Die Items bestehen aus ähnlich langen Sätzen und weisen einen vergleichbaren sprachlichen Komplexitätsgrad auf, so dass das Erraten erwünschter Antworten erschwert wird. Durch ein Expertenrating wurde sichergestellt, dass die Items von unabhängigen Beurteilern den theoretisch intendierten Kategorien zugeordnet werden. Dass über einzelne Formulierungen weiterhin diskutiert werden kann, bleibt davon unberührt und trifft vermutlich für jegliche Form psychologischer Tests zu. Aus Sicht der Geschichtswissenschaft und -didaktik ist auch ein solches Vorgehen nicht unproblematisch, denn die Items beinhalten Vermutungen, wie sich der Akteur in der speziellen historischen Situation fühlen könnte, was er denken und wie er handeln könnte. $\mathrm{Ob}$ diese Vermutungen den realen damaligen Handlungsalternativen entsprechen, ist nicht nachprüfbar. Zudem wird von Schülern mit einer solchen Methode nicht die Formulierung eigener, historisch plausibler Begründungen verlangt, sondern es geht um die Zustimmung zu relativ kurzen vorgefertigten Aussagen.

Beide Beispiele verdeutlichen die methodischen Schwierigkeiten, die sich vor allem in Details einer solchen interdisziplinären Untersuchung historischer Kompetenzen zeigen. Natürlich gibt es dafür Lösungsansätze, von denen einige in dieser Arbeit realisiert wurden. So wurden die Szenarien von Historikern (Experten der jeweiligen Epoche) auf ihre Plausibilität geprüft, die Items ebenfalls durch Expertenratings abgesichert und die Ratingskalenmethode ausgewählt um das Problem der einen richtigen Antwort zu umgehen. Zudem wurde das geschlossene Antwortformat durch eine weitere Frage ergänzt, die die Schüler schriftlich beantworten sollten. ${ }^{15}$ Die problematischen Aspekte scheinen aber Symptome eines grundlegenden Dilemmas zu sein, welches an die Oberfläche tritt, wenn historische Kompetenzen mit quantitativen und eher reduktionistischen Methoden der Psychologie erforscht werden. Es lässt sich bezweifeln, ob die Globalziele historisches Verstehen oder Geschichtsbewusstsein in ihrer Ganzheit als Kompetenzen im Rahmen von Bildungsstandards für das Fach Geschichte operationalisierbar gemacht werden können. Versteht man die bundesweite Debatte jedoch als Chance, durch die Erfassung von Teilkompetenzen historischen Verstehens auf die Potentiale des Geschichtsunterrichts hinzuweisen, liefert die vorliegende Studie einen ersten Beitrag, in dem Ideen aus der Psychologie und der Fachdidaktik zu einer fokussierten und ökonomisch einsetzbaren Methode kombiniert werden.

\footnotetext{
${ }^{15}$ Die schriftlichen Schülerantworten enthielten zu einem großen Teil Textreproduktion. Sie konnten deshalb nur selten bezogen auf das historische Perspektivenübernahmeniveau kodiert werden und blieben in den Analysen dieser Arbeit unberücksichtigt.
} 


\subsubsection{Von Kompetenzausprägungen zu Kompetenzstufen historischen Verstehens}

Mit der Verortung im Projekt „Kompetenzstufen historischen Verstehens“ und der theoretischen Anlehnung an das Entwicklungsstufenmodell von Selman (1980) zur sozialen Perspektivenkoordination bestand zumindest anfänglich der Anspruch, einen Entwurf für ein Stufenmodell historischer Perspektivenübernahme vorzulegen. Dieser Anspruch konnte in der vorliegenden Dissertation nicht eingelöst werden. Da ein solcher Anspruch prinzipiell für alle Teilkompetenzen historischen Verstehens gültig ist, wenn diese in Form von Bildungsstandards in die Schulrealität eingehen sollen, soll er an dieser Stelle vor dem Hintergrund der theoretischen und empirischen Erträge kritisch reflektiert werden.

Schüler unterscheiden sich in ihrer Kompetenz historische Perspektiven einzunehmen hinsichtlich ihrer Fixierung auf gegenwärtige Denkweisen und Wertmaßstäbe und hinsichtlich der Berücksichtigung des historischen Kontextes. Aufgrund dieser beiden Aspekte lassen sie sich als weniger kompetente bzw. kompetentere Schüler klassifizieren. Hinsichtlich der Bedeutung, die sie der Rolle und Funktion des historischen Akteurs zuschreiben, lassen sich zumindest die Siebtklässler voneinander unterscheiden. Die Relevanz dieses Aspekts für schulische Leistung im Fach Geschichte ist jedoch nicht unmittelbar abzuleiten. Mit diesen ersten Erkenntnissen liegen somit allenfalls zwei Endpunkte historischer Perspektivenübernahme vor. Wir wissen wenig über die Schritte, die zwischen Gegenwartsfixierung und historischer Kontextualisierung liegen. Von einem Modell, welches qualitative Entwicklungs- oder Lernprogressions-Stufen unterscheiden kann, ist die Forschung noch weit entfernt. Ashby und Lee (1987) liefern mit dem Vorschlag einer Hierarchie von Historical Empathy Ansatzpunkte für die Entwicklung dieser Kompetenz (vgl. Tabelle 1). Einschränkend ist dabei anzumerken, dass dieser Vorschlag auf der Auswertung von Gruppendiskussionen von Schülern einer Klassenstufe basiert. Die Aussagen sind also weder als individuelle Schülerleistungen interpretierbar noch sind sie auf andere Altersgruppen übertragbar.

Für den Anspruch, der an die Formulierung von Kompetenzstufen historischen Verstehens gestellt wird, haben die bisherigen Erkenntnisse mindestens zweierlei Konsequenzen. Erstens scheint die Entwicklung von Modellen, die Entwicklungsstufen historischen Verstehens abgekoppelt von Fortschritten in der sozial-kognitiven Entwicklung aufzeigen, weder theoretisch sinnvoll noch praktisch realisierbar. Theoretisch lässt sich der Gegenstand Geschichte als ein spezifischer Kontext verstehen, dessen Ver- 
ständnis mit großer Wahrscheinlichkeit von kognitiven Voraussetzungen wie der Fähigkeit zur sozialen Perspektivenübernahme, formal-operatorischen Denkstrukturen und anderen weitgehend universell ablaufenden Entwicklungsfortschritten abhängt. Dass diese universellen Strukturen durch fachspezifische Charakteristika ergänzt werden müssen um den Kontext angemessen abbilden zu können, scheint allein kein ausreichender Grund dafür zu sein, ein vollständig von psychologischen Theorien unabhängiges Entwicklungsstufenmodell historischen Verstehens aufzustellen, geschweige denn für einzelne Teilkompetenzen.

Eine zweite Konsequenz betrifft die Unterscheidung zwischen quantitativen und qualitativen Stufen. Hier sollte der Anspruch an Kompetenzmodelle und deren empirische Erfassung nicht zu stark heruntergeschraubt werden. Eine (willkürliche) Unterteilung in rein quantitative Kompetenzstufen aufgrund von Testpunktwerten läuft Gefahr, Zwischenschritte und Misskonzepte zu übersehen, die für die Entwicklung von Kompetenzen historischen Verstehens zentral sind und die im Geschichtsunterricht auf vielfältige Weise produktiv genutzt werden können. Das Verfahren der Latenten Klassenanalyse, welches in Kapitel 4 dieser Arbeit zur Anwendung kam, stellt dafür beispielsweise einen geeigneten methodischen Rahmen dar. Darüber hinaus sind qualitative Studien notwendig, um Kompetenzstufen aufzuzeigen, die sich im historischen Verstehen bei Schülern unterschiedlicher Altersstufen nachweisen lassen.

\subsection{Ausblick}

Die vorliegende Arbeit hat das Konzept der historischen Perspektivenübernahme im interdisziplinären Kontext von geschichtsdidaktischen Kompetenzmodellen und entwicklungs- und pädagogisch-psychologischen Theorien verortet und einen ersten Messzugang geschaffen. Bevor historische Perspektivenübernahme als Kompetenz im Rahmen von Bildungsstandards implementiert und größere Schülerstichproben daraufhin getestet werden können, sind Studien unabdingbar, die diese Kompetenz im Hinblick auf eine größere Bandbreite historischer Kontexte operationalisieren. Auch Untersuchungen zum Grad der Generalisierbarkeit über Kontexte hinweg sind wünschenswert. Weiterhin sollte überprüft werden, inwieweit sich das Messinstrument auch auf andere Schulformen anwenden lässt und welche Kompetenzunterschiede feststellbar sind. Dies 
ist insbesondere im Hinblick auf die Formulierung von Mindeststandards, wie sie von Klieme et al. (2003) gefordert werden, ein unerlässlicher nächster Schritt.

Ein mittelfristiges Ziel, welches die Weiterentwicklung der Messinstrumente voraussetzt, betrifft die Identifikation erfolgreicher Unterrichtskonzepte zur Förderung historischer Perspektivenübernahme bei Schülern. In der Debatte um Bildungsstandards wird es zwar oftmals als Vorteil dargestellt, dass Lehrenden alle Möglichkeiten offenstehen, Kompetenzen in individueller und kreativer Weise zu fördern. Genau deshalb sollte es jedoch mehr denn je Ziel der Fachdidaktik sein, empirisch gestützte Empfehlungen für einen kompetenzorientierten Unterricht bereitzustellen, und dies möglichst über allgemein geteilte Vermutungen wie „Rollenspiele fördern Perspektivenübernahme" hinaus.

Anknüpfen lässt sich ebenfalls an die Befunde aus Kapitel 5, dass soziale und historische Perspektivenübernahme in Klasse 7 positive Zusammenhänge aufweisen, diese jedoch in Klasse 10 nicht mehr bestehen. Weitere Studien, die diesem Befund (bestenfalls mit längsschnittlichen Designs) nachgehen, sind dringend notwendig. Denn der Bezug historischer Kompetenzen zur heutigen Lebenswelt der Schüler wird, wie in Kapitel 1 gezeigt wurde, von Wissenschaftlern und bildungspolitisch Verantwortlichen nahezu einstimmig gefordert und angenommen. Dies gilt nicht nur für die historische Perspektivenübernahme, sondern beispielsweise ebenfalls für den reflektierten Umgang mit historischen Quellen und Darstellungen, der sich positiv auf eine kritische Mediennutzung auswirken soll. Diese Brücke zwischen historischem Verstehen, sozialer Kompetenz sowie gesellschaftlich verantwortungsbewusstem Handeln stellt die Chance für das Fach Geschichte dar, sich in einer Bildungslandschaft, die von zunehmender Konzentration auf die Hauptfächer und die Naturwissenschaften geprägt ist, einen hohen Stellenwert zu sichern. Es fehlen noch zahlreiche Studien um Plädoyers wie das folgende empirisch zu untermauern:

We can learn from history how past generations thought and acted, how they responded to the demands of their time and how they solved their problems. We can learn by analogy, not by example, for our circumstances will always be different than theirs were. The main thing history can teach us is that human actions have consequences and that certain choices, once made, cannot be undone. They foreclose the possibility of making other choices and thus they determine future events (Lerner, 1997, p. 205). 


\section{Zusammenfassung}

Eine Kompetenz, die Schüler im Geschichtsunterricht erwerben sollen, ist das Hineinversetzen in die Gedanken- und Gefühlswelt von Menschen, die zu früheren Zeiten gelebt haben. Diese Kompetenz zur historischen Perspektivenübernahme wird in der vorliegenden Arbeit aus zwei Blickwinkeln beleuchtet: Zum einen werden fachdidaktische Kompetenzmodelle vorgestellt, in denen Perspektivenübernahme als integraler Bestandteil historischen Verstehens angesehen wird. Zum anderen wird auf das psychologische Modell zur sozialen Perspektivenkoordination von Robert L. Selman sowie auf empirische Befunde zu relevanten Kontextfaktoren zurückgegriffen. Das erste Forschungsziel betrifft die Entwicklung eines Messinstrumentes zur Erfassung historischer Perspektivenübernahme. Zweitens sollen mithilfe des neuen Messinstrumentes Beziehungen zwischen dieser fachspezifischen Kompetenz sowie geschichtsrelevanten und sozialkognitiven Schülermerkmalen untersucht werden. Die Annäherung an die Ziele erfolgt anhand von drei empirischen Originalarbeiten. Die Basis stellt eine Stichprobe von ca. 400 Schülern der 7. und 10. Klassenstufe an drei deutschen Gymnasien dar. Das Messinstrument besteht aus historischen Szenarien, zu denen Schüler insgesamt neun vierstufige Aussagen bewerten sollen, die drei Aspekte historischer Perspektivenübernahme abbilden (Gegenwartsfixierung, Rolle des historischen Akteurs, historische Kontextualisierung). Mittels Latenter Klassenanalyse können Schülertypen voneinander unterschieden werden. Während der überwiegenden Mehrheit der Zehntklässler eine Berücksichtigung des historischen Kontextes gelingt, überwiegt bei den Siebtklässlern die gegenwartsfixierte Betrachtung des historischen Szenarios. In einer nachfolgenden Analyse der korrelativen Zusammenhänge zwischen historischer Perspektivenübernahme, fachspezifischen Indikatoren wie Geschichtsinteresse, -selbstkonzept und -leistung sowie der Fähigkeit, im Alltag Perspektiven von Interaktionspartnern einzunehmen, ergeben sich ebenfalls unterschiedliche Befunde für die beiden Klassenstufen. Historische Perspektivenübernahme hängt bei den Siebtklässlern positiv mit der Fähigkeit zur sozialen Perspektivenübernahme zusammen. In der 10. Klasse ist diese Kompetenz historischen Verstehens eingebettet in das Gefüge aus fachspezifischen Merkmalen. Am Ende der vorliegenden Arbeit stehen eine Arbeitsdefinition von historischer Perspektivenübernahme, ein Vorschlag für ein Messinstrument sowie weiterführende Fragen, die die Entwicklung dieser Kompetenz im Zusammenspiel mit fachspezifischen und sozialkognitiven Motivations- und Leistungsindikatoren betreffen. 


\section{Abstract (english)}

A competency that students should acquire in learning history is inferring thoughts and feelings of people who have lived in past times. This competency called historical perspective taking is the focus of this dissertation and will be observed from two angles: First, I will review didactical models which conceptualize perspective taking as an integral part of historical thinking. Then, I will describe Robert L. Selman's psychological theory of social perspective coordination, and will add some empirical findings on factors that may influence the development of such a competency. The first objective of the present work is the development of a new measure to assess historical perspective taking. Using that measure, my second objective is to gain insights into the relationships between this historical competency and other domain-specific as well as socio-cognitive attributes. To achieve these aims, I present three pieces of empirical work. Approximately 400 students from $7^{\text {th }}$ and $10^{\text {th }}$ grade of three German grammar schools are included in the study. The measure for historical perspective taking consists of historical scenarios and nine statements that students are supposed to rate on a four-point rating scale. These statements represent three aspects of historical perspective taking (presentism, role of the historical agent, contextualization). Using latent class analysis, different types of students can be identified. While the vast majority of $10^{\text {th }}$ graders is able to consider the historical context of the scenario, presentist judgments prevail in the responses of the $7^{\text {th }}$ graders. In a subsequent correlative analysis that aims at clarifying relations between historical perspective taking, domain-specific indicators (interest for history, self-concept, and achievement), and the ability to take perspectives of other people in everyday situations, I also noticed major differences between students of grade 7 and 10 . For $7^{\text {th }}$ graders, historical perspective taking was positively related to the ability to take social perspectives in everyday situations. For $10^{\text {th }}$ graders, historical perspective taking seemed to be embedded in the network of history-specific characteristics in students. At the end of this work, I present a working definition of historical perspective taking and a suggestion for an approach to measure this competency. I close with some further questions about the development of the competency to take historical perspectives within the interplay of domain-specific and socio-cognitive indicators of motivation and achievement. 


\section{Literatur}

Adalbjarnardottir, S. (1995). How schoolchildren propose to negotiate: The role of social withdrawal, social anxiety, and locus of control. Child Development, 66, 1739-1751.

Angvik, M. \& Borries, B. von (1997). Youth and history: A comparative European survey on historical consciousness and political attitudes among adolescents. Hamburg: Körber-Stiftung.

Ashby, R. \& Lee, P. (1987). Children's concepts of empathy and understanding in history. In C. Portal (Ed.), The history curriculum for teachers (pp. 62-88). London: The Falmer Press.

Baring, F. (2004). Internationale geschichtsdidaktische Perspektiven - Multiperspektivität, Empathie und Perspektivenübernahme in den USA und Großbritannien. In S. Handro \& B. Schönemann (Hrsg.), Geschichtsdidaktische Lehrplanforschung: Methoden - Analysen - Perspektiven (203-214). Münster: LIT Verlag.

Barton, K. C. (1996). Narrative simplifications in elementary students' historical thinking. Advances in Research on Teaching, 6, 51-83.

Bayrhuber, H., Bögeholz, S., Eggert, S., Elster, D., Grube, C., Hößle, C. et al. (2007). Biologie im Kontext (bik) - Erste Forschungsergebnisse zu den Kompetenzbereichen Fachwissen, Erkenntnisgewinnung, Kommunikation \& Bewertung sowie zur Evaluation des Projekts. Der Mathematische und Naturwissenschaftliche Unterricht (MNU), 60/5, 304-313.

Beardslee, W. R., Hickey Schultz, L. \& Selman, R. L. (1987). Level of social-cognitive development, adaptive functioning, and DSM-III diagnoses in adolescent offspring of parents with affective disorders: Implications of the development of the capacity for mutuality. Developmental Psychology, 23, 807-815.

Beck, I. L. \& McKeown, M. G. (1994). Outcomes of history instruction: Paste-up accounts. In M. Carretero \& J. F. Voss (Eds.), Cognitive and Instructional Processes in History and the Social Sciences (pp. 237-256). Hillsdale, NJ: Erlbaum.

Bergmann, K. (2000). Multiperspektivität. Geschichte selber denken. Schwalbach am Taunus: Wochenschau Verlag.

Bermúdez, A. \& Jaramillo, R. (2001). Development of historical explanation in children, adolescents and adults. In A. Dickinson, P. Gordon \& P. Lee (Eds.), Rai- 
sing standards in history education (Vol. 3, pp. 146-167). London: Woburn Press.

Bernhardt, M. (2007). Die Subjektseite der visuellen Begegnung. Vom Nutzen qualitativer empirischer Untersuchungen für die Entwicklung fachspezifischer Kompetenzen. Zeitschrift für Geschichtsdidaktik, 108-124.

Borries, B. von (1994). (Re-)constructing history and moral judgement: On relationships between interpretations of the past and perceptions of the present. In M. Carretero \& J. F. Voss (Eds.), Cognitive and instructional processes in history and the social sciences (pp. 339-355). Hillsdale, NJ: Erlbaum.

Borries, B. von (1995). Das Geschichtsbewusstsein Jugendlicher. Eine repräsentative Untersuchung über Vergangenheitsdeutungen, Gegenwartswahrnehmungen und Zukunftserwartungen von Schülerinnen und Schülern in Ost- und Westdeutschland. Weinheim: Juventa.

Borries, B. von (2007). Empirie: Ergebnisse messen (Lerndiagnose im Fach Geschichte). In A. Körber, W. Schreiber \& A. Schöner (Hrsg.), Kompetenzen historischen Denkens. Ein Strukturmodell als Beitrag zur Kompetenzorientierung im Fach Geschichte (S. 653-673). Neuried: ars una.

Caldarella, P. \& Merell, K. W. (1997). Common dimensions of social skills of children and adolescents: A taxonomy of positive behaviours. School Psychology Review, $26,264-278$.

Chomsky, N. (1968). Language and mind. New York: Harcourt, Brace \& World.

Colby, A., Kohlberg, L., Gibbs, J. \& Lieberman, M. (1983). A longitudinal study of moral judgment. Monographs of the Society for Research in Child Development, 48 (1-2, Serial No. 200), 1-96.

Davier, M. von (1997). Bootstrapping goodness-of-fit statistic for sparse categorial data - Results of a monte carlo study. Methods of Psychological Research Online, 2, $29-48$.

Davier, M. von (2001). WINMIRA 2001 [computer software].

Davis, M. H. (1983a). The effects of dispositional empathy on emotional reactions and helping: A multidimensional approach. Journal of Personality, 51, 167-184.

Davis, M. H. (1983b). Measuring individual differences in empathy: Evidence for a multidimensional approach. Journal of Personality and Social Psychology, 44, 113-126. 
Davis, O. L., Jr. (2001). In pursuit of historical empathy. In O. L. Davis Jr., E. A. Yeager \& S. J. Foster (Eds.), Historical empathy and perspective taking in the social studies (pp. 1-12). Lanham, MD: Rowman \& Littlefield.

Davis, O. L., Jr., Yeager, E. A. \& Foster, S. J. (Eds.). (2001). Historical empathy and perspective taking in the social studies. Lanham, MD: Rowman \& Littlefield.

Denissen, J. J. A., Zarrett, N. R. \& Eccles, J. S. (2007). I like to do it, I'm able, and I know I am: Longitudinal couplings between domain-specific achievement, selfconcept, and interest. Child Development, 78, 430-447.

Department for Education and Employment \& Qualifications and Curriculum Authority (1999). National Curriculum for History - Online Version.

(http://www.nc.uk.net/nc_resources/html/download.shtml). gelesen am 23.4.2008.

Dickinson, A., Gordon, P. \& Lee, P. (Eds.). (2001). Raising standards in history education (Vol. 3). London: Woburn Press.

Eisenberg, N., Murphy, B. C. \& Shepard, S. (1997). The development of empathic accuracy. In W. Ickes (Ed.), Empathic accuracy (pp. 73-116). New York: Guilford Press.

Feigenberg, L. F., King, M. S., Barr, D. J. \& Selman, R. L. (2008). Belonging to and exclusion from the peer group in schools: Influences on adolescents' moral choices. Journal of Moral Education, 37, 165-184.

Foster, S. J. (2001). Historical empathy in theory and practice: Some final thoughts. In O. L. Davis Jr., E. A. Yeager \& S. J. Foster (Eds.), Historical empathy and perspective taking in the social Studies (pp. 167-181). Lanham, MD: Rowman \& Littlefield.

Gautschi, P. (2006). Kompetenzmodell für den Geschichtsunterricht. (http://hinschauenundnachfragen.ch/3kompetenzen.html). gelesen am 27.2.2008.

Gautschi, P., Moser, D. V., Reusser, K. \& Wiher, P. (Hrsg.). (2007). Geschichtsunterricht heute. Eine empirische Analyse ausgewählter Aspekte. Bern: h.e.p. Verlag.

Gehlbach, H. (2004a). Social perspective taking: A facilitating aptitude for conflict resolution, historical empathy, and social studies achievement. Theory and Research in Social Education, 32, 39-55.

Gehlbach, H. (2004b). A new perspective on perspective taking: A multidimensional approach to conceptualizing an aptitude. Educational Psychology Review, 16, 207-234. 
Gehlbach, H. (2006). How changes in students' goal orientations relate to outcomes in social studies. The Journal of Educational Research, 99, 358-370.

Günther-Arndt, H. \& Sauer, M. (Hrsg.). (2006). Geschichtsdidaktik empirisch. Untersuchungen zum historischen Denken und Lernen. Berlin: LIT Verlag.

Gurucharri, C. \& Selman, R. L. (1982). The development of interpersonal understanding during childhood, preadolescence and adolescence: A longitudinal follow-up study. Child Development, 53, 924-927.

Gutzwiller-Helfenfinger, E. (2003). Assessing social perspective-taking in adolescence: The written interpersonal understanding interview. Dissertation, Universität Bern. Hartmann, U. (im Druck). Kompetenzprofile historischer Perspektivenübernahme in Klasse 7 und 10. In J. Hodel \& B. Ziegler (Hrsg.), Forschungswerkstatt Geschichtsdidaktik empirisch 07. Bern: h.e.p. Verlag.

Hartmann, U. \& Hasselhorn, M. (2008). Historical perspective taking - A standardized measure for an aspect of students' historical thinking. Learning and Individual Differences, 18, 264-270.

Hartmann, U., Martens, M. \& Sauer, M. (2007). Von Kompetenzmodellen zur empirischen Erforschung von Schülerkompetenzen - das Beispiel historische Perspektivenübernahme. Zeitschrift für Geschichtsdidaktik. S. 125 - 148.

Hartmann, U., Sauer, M. \& Hasselhorn, M. (im Druck). Perspektivenübernahme als Kompetenz für den Geschichtsunterricht: theoretische und empirische Zusammenhänge zwischen fachspezifischen und sozial-kognitiven Schülermerkmalen. Zeitschrift für Erziehungswissenschaft.

Hasberg, W. \& Körber, A. (2003). Geschichtsbewusstsein dynamisch. In A. Körber (Hrsg.), Geschichte - Leben - Lernen. Bodo von Borries zum 60. Geburtstag (S. 179-202). Schwalbach am Taunus: Wochenschau Verlag.

Heller, K. A. \& Perleth, C. (2000). Kognitiver Fähigkeits-Test (KFT 4-12+R) (3. Aufl.). Göttingen: Beltz Testgesellschaft.

Hickey Schultz, L., Barr, D. J. \& Selman, R. L. (2001). The value of a developmental approach to evaluating character development programmes: an outcome study of Facing History and Ourselves. Journal of Moral Education, 30, 3-27.

Hickey Schultz, L. \& Selman, R. L. (2004). The development of psychological maturity in young children - A measure for evaluating character education programs. Journal of Research in Character Education, 2, 19-43. 
Hodel, J. \& Ziegler, B. (Hrsg.) (im Druck). Forschungswerkstatt Geschichtsdidaktik empirisch 07. Bern: h.e.p. Verlag.

Ickes, W. (Ed.). (1997). Empathic accuracy. New York: Guilford Press.

Ickes, W., Stinson, L., Bissonnette, V. \& Garcia, S. (1990). Naturalistic social cognition: Empathic accuracy in mixed-sex dyads. Journal of Personality and Social Psychology, 59, 730-742.

Jeismann, K.-E. (1980). 'Geschichtsbewußtsein'. Überlegungen zur zentralen Kategorie eines neuen Ansatzes der Geschichtsdidaktik. In H. Süssmuth (Hrsg.), Geschichtsdidaktische Positionen. Bestandsaufnahme und Neuorientierung (S. 179222). Paderborn: Schöningh/UTB.

Jerusalem, M. \& Klein-Heßling, J. (2002). Soziale Kompetenz. Entwicklungstrends und Förderung in der Schule. Zeitschrift für Psychologie, 210, 164-174.

Johnson, D. W. (1975). Cooperativeness and social perspective taking. Journal of Personality and Social Psychology, 31, 241-244.

Kaufhold, M. (2006). Kompetenz und Kompetenzerfassung: Analyse und Beurteilung von Verfahren der Kompetenzerfassung. Wiesbaden: VS Verlag für Sozialwissenschaften.

Keller, M. \& Edelstein, W. (1991). The development of social-moral meaning making: Domains, categories, and perspective-taking. In W. M. Kurtines \& J. L. Gewirtz (Eds.), Handbook of moral behavior and development: Vol. 2. Research (pp. 89114). Hillsdale, NJ: Erlbaum.

Keller, M., Edelstein, W., Krettenauer, T., Fu-xi, F. \& Ge, F. (2005). Reasoning about moral obligations and interpersonal responsibilities in different cultural contexts. In W. Edelstein \& G. Nunner-Winkler (Eds.), Morality in context (pp. 317-337). Amsterdam: Elsevier.

Keller, M., Lourenco, O., Malti, T. \& Saalbach, H. (2003). The multifacetted phenomenon of 'happy victimizers': A cross-cultural comparison of moral emotions. British Journal of Developmental Psychology, 21, 1-18.

Klieme, E. \& Hartig, J. (2007). Kompetenzkonzepte in den Sozialwissenschaften und im erziehungswissenschaftlichen Diskurs. Zeitschrift für Erziehungswissenschaft, Sonderheft 8, 11-29.

Klieme, E., Avenarius, H., Blum, W., Döbrich, P., Gruber, H., Prenzel, M. et al. (2003). Expertise zur Entwicklung nationaler Bildungsstandards. Berlin: BMBF. 
Kölbl, C., Tiedemann, J. \& Billmann-Mahecha, E. (2006). Die Bedeutung der Lesekompetenz für Sachfächer. Psychologie in Erziehung und Unterricht, 53, 201212.

Körber, A., Meyer-Hamme, J. \& Schreiber, W. (2007). Überlegungen zu Graduierungslogiken der Kernkompetenzen im Kompetenzbereich Historische Orientierungskompetenzen. In A. Körber, W. Schreiber \& A. Schöner (Hrsg.), Kompetenzen historischen Denkens. Ein Strukturmodell als Beitrag zur Kompetenzorientierung in der Geschichtsdidaktik (S. 473-504). Neuried: ars una.

Körber, A., Schreiber, W. \& Schöner, A. (Hrsg.). (2007). Kompetenzen historischen Denkens. Ein Strukturmodell als Beitrag zur Kompetenzorientierung in der Geschichtsdidaktik. Neuried: ars una.

Kuhn, D., Weinstock, M. \& Flaton, R. (1994). Historical reasoning as theory-evidence coordination. In M. Carretero \& J. F. Voss (Eds.), Cognitive and instructional processes in history and the social sciences (pp. 377-402). Hillsdale, NJ: Erlbaum.

Kultusministerium Baden-Württemberg (Hrsg.). (2004). Bildungsstandards für Geschichte, Gymnasium - Klassen 6, 8, 10 (http://www.bildung-staerktmenschen.de/service/downloads/Bildungsstandards/Gym/Gym_G_bs.pdf). gelesen am 25.4.2008.

Kultusministerkonferenz (2005). Einheitliche Prüfungsanforderungen in der Abiturprüfung GESCHICHTE. Beschluss vom 10.2.2005.

Kunter, M., Schümer, G., Artelt, C., Baumert, J., Klieme, E. Neubrand, M. et al. (2002). PISA 2000: Dokumentation der Erhebungsinstrumente. Materialien aus der Bildungsforschung, 72. Berlin.

Lazarsfeld, P. F. \& Henry, N. W. (1968). Latent structure analysis. Boston: Houghton Mill.

Lee, P. \& Ashby, R. (2000). Progression in historical understanding among students ages 7-14. In P. N. Stearns, P. Seixas \& S. Wineburg (Eds.), Knowing, teaching, and learning history - National and international perspectives (pp. 199-222). New York: University Press.

Lee, P. \& Ashby, R. (2001). Empathy, perspective taking, and rational understanding. In O. L. Davis Jr., E. A. Yeager \& S. J. Foster (Eds.), Historical empathy and perspective taking in the social studies (pp. 21-50). Lanham, MD: Rowman \& Littlefield. 
Lee, P., Dickinson, A. \& Ashby, R. (1998). Researching children's ideas about history.

In J. F. Voss \& M. Carretero (Eds.), Learning and reasoning in history (pp. 227251). London: Woburn Press.

Lerner, G. (1997). Why history matters: Life and thought. New York: Oxford University Press.

Martens, M. (2008). Geschichtsunterricht als Ort historischen Lernens: Wie gehen Schülerinnen und Schüler mit Darstellungen von Vergangenheit um? In S. Handro \& B. Schönemann (Hrsg.), Orte historischen Lernens (S. 61-73). Berlin: LIT Verlag.

Mischo, C. (2005). Promoting perspective coordination by dilemma discussion. The effectiveness of classroom group discussion on interpersonal negotiation strategies of 12-year-old students. Social Psychology of Education, 8, 41-63.

National Center for History in the Schools (1996). National Standards for History. Basic Edition. Los Angeles, CA.

Niedersächsisches Kultusministerium (Hrsg.). (2004). Curriculare Vorgaben für das Gymnasium, Schuljahrgänge 5/6 Geschichte.

(http://db2.nibis.de/1db/cuvo/datei/5_geschichte_gym_5.pdf). gelesen am 25.4.2008.

Pandel, H.-J. (1987). Dimensionen des Geschichtsbewusstseins. Ein Versuch, seine Struktur für Empirie und Pragmatik diskutierbar zu machen. Geschichtsdidaktik, $12,130-142$.

Pandel, H.-J. (2005). Geschichtsunterricht nach PISA. Kompetenzen, Bildungsstandards und Kerncurricula. Schwalbach am Taunus.: Wochenschau Verlag.

Piaget, J. (1970). Structuralism. New York: Basic Books.

Portal, C. (1987). Empathy as an objective for history teaching. In C. Portal (Ed.), The history curriculum for teachers (pp. 89-99). London: The Falmer Press.

Prenzel, M., Artelt, C., Baumert, J., Blum, W., Hammann, M., Klieme, E. \& Pekrun, R. (Hrsg.) (2007). PISA 2006. Die Ergebnisse der dritten internationalen Vergleichsstudie. Münster: Waxmann.

Quintana, S. B., Ybarra, V. C., Gonzalez-Doupe, P. \& De Baess, Y. (2000). Crosscultural evaluation of ethnic perspective-taking ability: An exploratory investigation with U.S. Latino and Guatemalan Ladino children. Cultural Diversity and Ethnic Minority Psychology, 6, 334-351. 
Rost, D. H. \& Sparfeldt, J. R. (2002). Facetten des schulischen Selbstkonzepts. Ein Verfahren zur Messung des differentiellen Selbstkonzepts schulischer Leistungen und Fähigkeiten (DISK-Gitter). Diagnostica, 48, 130-140.

Royzman, E. B., Wright Cassidy, K. \& Baron, J. (2003). "I know, you know": Epistemic egocentrism in children and adults. Review of General Psychology, 7, 3865.

Rüsen, J. (1983). Historik und Didaktik. Ort und Funktion der Geschichtstheorie im Zusammenhang von Geschichtsforschung und historischer Bildung. In E. Kosthorst (Hrsg.), Geschichtswissenschaft. Didaktik - Forschung - Theorie (S. 4864). Göttingen: Vandenhoeck \& Ruprecht.

Sauer, M. (2002). Methodenkompetenz als Schlüsselqualifikation. Eine neue Grundlegung des Geschichtsunterrichts? Geschichte, Politik und ihre Didaktik, 30, 183192.

Sauer, M. (2006). Kompetenzen für den Geschichtsunterricht - ein pragmatisches Modell als Basis für die Bildungsstandards des Verbandes der Geschichtslehrer. Informationen für den Geschichts- und Gemeinschaftskundelehrer, 72, 7-20.

Sauer, M., Martens, M., Hartmann, U. \& Hasselhorn, M. (Hrsg.). (in Vorbereitung). Interpersonal understanding in historical context. Rotterdam: Sense Publishers.

Schreiber, W. (1999). Die Entwicklung historischer Sinnbildungskompetenzen als Ziel des historischen Lernens mit Grundschülern. In W. Schreiber: Erste Begegnungen mit Geschichte. Grundlagen historischen Lernens (S. 15-79). Neuried: ars una.

Schreiber, W. (2007). Kompetenzbereich Historische Orientierungskompetenzen. In A. Körber, W. Schreiber \& A. Schöner (Hrsg.), Kompetenzen historischen Denkens. Ein Strukturmodell als Beitrag zur Kompetenzorientierung in der Geschichtsdidaktik (S. 236-264). Neuried: ars una.

Schreiber, W. (2008). Ein Kompetenz-Strukturmodell historischen Denkens. Zeitschrift für Pädagogik, 54, 198-212.

Schreiber, W., Körber, A., Borries, B., von, Krammer, R., Leutner-Ramme, S., Mebus, S. et al. (2007). Historisches Denken. Ein Kompetenz-Strukturmodell. In A. Körber, W. Schreiber \& A. Schöner (Hrsg.), Kompetenzen historischen Denkens. Ein Strukturmodell als Beitrag zur Kompetenzorientierung in der Geschichtsdidaktik (S. 17-53). Neuried: ars una. 
Seixas, P. (1996). Conceptualizing the growth of historical understanding. In D. Olsen \& N. Torrance (Eds.), Handbook of education and human development: New models of learning, teaching, and schooling (pp. 765-783). Oxford UK: Blackwell.

Selman, R. L. (1980). The growth of interpersonal understanding - Developmental and clinical analyses. New York: Academic Press.

Selman, R. L. (2003). The promotion of social awareness. Powerful lessons from the partnership of developmental theory and classroom practice. New York: Russell Sage Foundation.

Senatsverwaltung für Bildung, Jugend und Sport Berlin (Hrsg.). (2006a). Rahmenlehrplan für die Sekundarstufe 1 Jahrgangsstufe 7-10 Geschichte.

Senatsverwaltung für Bildung, Jugend und Sport Berlin (Hrsg.). (2006b). Rahmenlehrplan für die gymnasiale Oberstufe Geschichte.

Shemilt, D. (1980). History 13 - 16 evaluation study. Edinburgh: Holmes McDougall.

Shemilt, D. (1987). Adolescent ideas about evidence and methodology in history. In C. Portal (Ed.), The history curriculum for teachers (pp. 39-61). London: The Falmer Press.

Smetana, J. G. (2006). Social-cognitive domain theory: Consistencies and variations in children's moral and social judgments. In M. Killen \& J. G. Smetana (Eds.), Handbook of moral development (pp. 119-154). Hillsdale, NJ: Erlbaum.

Smetana, J. G., Killen, M. \& Turiel, E. (1991). Children's reasoning about interpersonal and moral conflicts. Child Development, 62, 629-644.

Snow, R. E. (1996). Aptitude development and education. Psychology, Public Policy, and Law, 2, 536-560.

Spoehr, K. T. \& Spoehr, L. W. (1994). Learning to think historically. Educational Psychologist, 29, 71-77.

Stearns, P. N., Seixas, P. \& Wineburg, S. (Eds.). (2000). Knowing, teaching and learning history - National and international perspectives. New York: University Press.

Steins, G. (1998). Diagnostik von Empathie und Perspektivenübernahme: Eine Überprüfung des Zusammenhangs beider Konstrukte und Implikationen für die Messung. Diagnostica, 44, 117-129.

Stodolsky, S. S., Salk, S. \& Glaessner, B. (1991). Student views about learning math and social studies. American Educational Research Journal, 28, 89-116. 
Tenorth, H.-E. (2008). Bildungsstandards außerhalb der Kernfächer. Herausforderungen für den Unterricht und die fachdidaktische Forschung - Zur Einleitung in den Thementeil. Zeitschrift für Pädagogik, 54, 159-162.

Turiel, E. (1983). Domains and categories in social cognitive development. In W. Overton (Ed.), The relationship between social and cognitive development (pp. 5390). Hillsdale, NJ: Erlbaum.

VanSledright, B. A. (2001). From empathic regard to self-understanding: Im/Positionality, empathy, and historical contextualization. In O. L. Davis Jr., E. A. Yeager \& S. J. Foster (Eds.), Historical empathy and perspective taking in the social studies (pp. 51-68). Lanham, MD: Rowman \& Littlefield.

Verband der Geschichtslehrer Deutschlands e.V. (2006). Bildungsstandards Geschichte. Rahmenmodell Gymnasium 5.-10. Jahrgangsstufe.

Voss, J. F. \& Carretero, M. (Eds.). (1998). Learning and reasoning in history. London: Woburn Press.

Voss, J. F. \& Wiley, J. (1997). Conceptual understanding in history. European Journal of Psychology of Education, 12(3), 147-158.

Wainryb, C. (1993). The application of moral judgments to other cultures: Relativism and universality. Child Development, 64, 924-933.

Weinert, F. E. (2001). Vergleichende Leistungsmessung in Schulen - eine umstrittene Selbstverständlichkeit. In F. E. Weinert (Hrsg.), Leistungsmessungen in Schulen (S. 17-31). Weinheim: Beltz.

Wiley, J. \& Voss, J. F. (1999). Constructing arguments from multiple sources: tasks that promote understanding and not just memory for text. Journal of Educational Psychology, 91, 301-311.

Wineburg, S. (1991). Historical problem solving: A study of the cognitive processes used in the evaluation of documentary and pictorial evidence. Journal of Educational Psychology, 83, 73-87.

Wineburg, S. (1999). Historical thinking and other unnatural acts. Phi Delta Kappan, $80,488-499$.

Wirtz, M. \& Caspar, F. (2002). Beurteilerübereinstimmung und Beurteilerreliabilität. Göttingen: Hogrefe.

Wolters, C. A. \& Pintrich, P. R. (1998). Contextual differences in student motivation and self-regulated learning in mathematics, English, and social studies classrooms. Instructional Science, 26, 27-47. 
Yeager, E. A. \& Foster, S. J. (2001). The role of empathy in the development of historical understanding. In O. L. Davis Jr., E. A. Yeager \& S. J. Foster (Eds.), Historical empathy and perspective taking in the social studies (pp. 13-20). Lanham, MD: Rowman \& Littlefield.

Yeates, K. O. \& Selman, R. L. (1989). Social competence in the schools: Toward an integrative developmental model for intervention. Developmental Review, 9, 64100. 


\section{Anhang}

A.1: Election Scenario (English Version)

A.2: Kloster-Szenario

A.3: Ausschnitt des Messinstrumentes - identischer Teil für die Klassenstufen 7 und 10

A.4: Multiple Choice-Items und Lückentext für Klassenstufe 7

A.5: Multiple Choice-Items und Lückentext für Klassenstufe 10 


\section{A.1}

\section{Election scenario}

Düsseldorf, Germany in 1930. Hannes (20 years old) is the son of a man who owns a small business manufacturing tailor-made shoes. One day Hannes meets with his friend Gerd. They talk about the situation in Germany and the upcoming elections.

Hannes says: "My father's company is teetering on the brink of collapse. Since the war ended, everything is getting worse and worse. After the depression in 1923, we began to feel some hope again. But now it is worse than ever before. I don't know how this is going to end. Right now, I still have a job in my father's business. But when he closes down, I have no idea where to get a job. We have always been reputable people - and look at us now!"

Gerd responds: "You are right. What has happened to our country? Look at what is going on today. Nobody has a job and the mobs in the streets are letting loose on each other."

Hannes replies: "My father always says that we were better off during the time of the empire. What can we do when our country suffers from a crisis and the winners of the war are hurting us wherever they can? Our politicians with their policy of appeasement really don't do us any good. It is high time for Germany to be ruled by someone who knows what to do and who really takes the lead. During the last election, I supported the DNVP (conservative nationalistic party in Germany during that time), but I don't know if they've got the right people to save our country."

Below you will find some statements. Read through all the statements first. Then, try to take Hannes' perspective and mark for every statement how well it fits his situation.

Could Hannes vote for an anti-democratic party like the NSDAP?

$\begin{array}{cccc}\text { Doesn't } & \text { Doesn't } & \text { Fits his } & \text { Fits his } \\ \text { fit his } & \text { fit his } & \text { situation } & \text { situation } \\ \text { situation } & \text { situation } & \text { somewhat } & \text { very } \\ \text { at all } & \text { too } & & \text { well } \\ & \text { much } & & \end{array}$

POP1 He certainly won't vote for the NSDAP. What they have done to Germany and the world, one cannot approve.

POP2 He will realize that only in a democracy can people take part in decision-making. That is why he will decide reasonably, and that does not mean NSDAP.

POP3 He will not vote for the NSDAP. Their slogans are easy to see through. It is evident that this will lead to war.

ROA1 As a member of the bourgeoisie he would proba- 
bly like to go back to the empire when his family was better off. He could vote for an antidemocratic party.

ROA2 As a son of a businessman he will likely vote for a conservative party, but not necessarily for the NSDAP.

ROA3 Because his father's company is about to collapse, he could vote for a party that represents the rights of the smaller businessmen.

CONT1 Hannes lacks experience with democracy. He probably doesn't know about the risk connected with the NSDAP, and thus is likely to vote for them.

CONT2 To him, Hitler probably represents a strong leader. He probably won't think too much about the threats connected with the NSDAP.

CONT3 In his situation he only sees the disadvantages of democracy. That is why he is likely to fall for the slogans of the NSDAP.

Notes:

Items are placed in order of the category of historical perspective taking. In the original instrument, items were placed in random order. Items 1 to 3 contain present-oriented perspective taking (POP); items 4 to 6 contain the role of the historical agent (ROA); items 7 to 9 contain historical contextualization (CONT). 


\section{Kloster-Szenario}

Die 22jährige Johanna ist Tochter des Freiherrn von Weinburg. Seit einiger Zeit sind Gespräche über ihre Verheiratung mit Karl, dem Sohn des Freiherrn von Schwanstein, im Gang. Johanna kennt Karl zwar noch nicht gut, sie möchte aber gerne heiraten und eine Familie gründen. Als Johannas Vater sie zu einem Gespräch nach dem Mittagsmahl ruft, denkt sie, dass er ihr den genauen Hochzeitstermin mitteilen will. Aber es kommt anders:

„Mein liebes Kind, ich muss ernst mit dir reden. Karl von Schwanstein wird sich nicht mit dir sondern mit einer anderen vermählen. Er wird die Tochter des Grafen von Falkeneck heiraten. Ihre Familie ist wohlhabender als die unsrige. Deshalb hat er sich für sie entschieden.“

Johanna ist erschrocken und antwortet: „Aber Vater, welch schreckliche Nachricht bringt Ihr mir. Unsere Vermählung schien mir so sicher! Was soll nun aus mir werden?“”

„Ich habe lange überlegt und mich mit deiner Mutter beraten. Eine Vermählung in deinem Alter wird kaum noch möglich sein. Das Beste für uns alle wird sein, wenn du den Schleier nimmst ${ }^{16}$ und ins Kloster Lichtenthal eintrittst. Ein gottgefälliges Leben, wie es das Kloster bietet, wird allen Weinburgern das Seelenheil bringen. Schon bald wirst du von der Äbtissin ${ }^{17}$ feierlich aufgenommen und wirst ihr das Versprechen von Armut, Ehelosigkeit und Gehorsam geben.“

Unten sind einige Aussagen aufgelistet. Lies dir zuerst alle Aussagen einmal durch. Versuch dich in Johanna hineinzuversetzen und kreuze für jede Aussage an, wie gut sie deiner Meinung nach zu ihrer Situation passt.

Was bedeutet es für Johanna, wenn ihre Eltern sie ins Kloster schicken?

$\begin{array}{cccc}\text { Passt } & \text { Passt } & \text { Passt } & \text { Passt } \\ \text { gar } & \text { eher } & \text { ziem- } & \text { voll und } \\ \text { nicht zu } & \text { nicht zu } & \text { lich zu } & \text { ganz zu } \\ \text { ihrer } & \text { ihrer } & \text { ihrer } & \text { ihrer } \\ \text { Situa- } & \text { Situa- } & \text { Situa- } & \text { Situa- } \\ \text { tion } & \text { tion } & \text { tion } & \text { tion }\end{array}$

GEG1 Für Johanna muss es furchtbar sein, ins Kloster zu gehen, weil sie dann keine Chance mehr hat, jemanden kennen zu lernen, den sie liebt.

GEG2 Sie wird ihre Eltern grausam finden, weil sie sie in ein Kloster geben und ihr keine Möglichkeit geben zu heiraten.

GEG3 Johanna wird sich von ihren Eltern schlecht behandelt fühlen, weil sie ihr

\footnotetext{
${ }^{16}$ Mittelalterliche Redewendung für „ins Kloster eintreten“

${ }^{17}$ Vorsteherin des Klosters
} 
nicht ermöglichen, ein eigenes, selbstbestimmtes Leben zu führen.

ROLLE1 Johanna kann als Tochter nichts gegen die Entscheidung ihrer Eltern sagen und muss sich so verhalten, wie sie es wollen.

ROLLE2 Johanna versteht, dass Karl lieber die Tochter eines Grafen heiraten möchte.

ROLLE3 Johanna muss sich als Tochter dem Willen ihrer Eltern beugen.

KONT1 Auf die Heirat zu verzichten ist für Johanna nicht so schlimm. Für sie ist vor allem wichtig, dass sie für ihr Leben versorgt ist.

KONT2 Johanna weiß, dass ihre Eltern sie mit guten Absichten ins Kloster geben, damit sie auch als unverheiratete Frau versorgt ist.

KONT3 Religion spielt für Johanna eine ganz andere Rolle als für viele Menschen heute. Deshalb ist es für sie sinnvoll, ein Leben im Kloster zu führen.

Anmerkungen:

In der Originalaufgabe waren die Items in zufälliger Reihenfolge angeordnet. Erläuterung der Kategorien: $G E G=$ Gegenwartsfixierung $;$ ROLLE $=$ Rolle des historischen Akteurs $;$ KONT $=$ Historische Kontextualisierung. 


\section{A.3}

Ausschnitt des Messinstrumentes - identischer Teil für Klassenstufen 7 und 10

\section{Befragung für Schülerinnen und Schüler im Geschichtsunterricht}
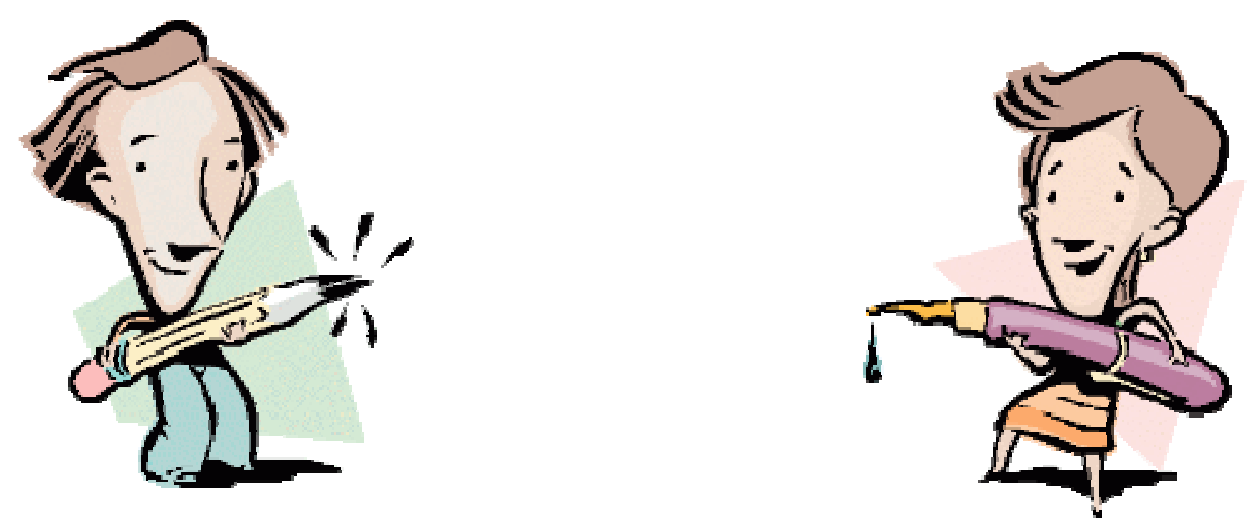

Deine Meinung ist gefragt!

In welche Klasse gehst du?

Alter: Jahre

Bist du ein Mädchen oder ein Junge?

$\square$ Mädchen

$\square$ Junge

Wir wënschen dir viel Spaß Geim Ansfüllen! 


\section{Du bekommst in den nächsten zwei Schulstunden einige Fragen gestellt.}

Hier sind zwei Beispiele für Fragen:

Beispiel 1:

\begin{tabular}{|l|c|c|c|c|}
\hline & $\begin{array}{c}\text { trifft gar } \\
\text { nicht zu }\end{array}$ & $\begin{array}{c}\text { trifft eher } \\
\text { nicht zu }\end{array}$ & trifft eher zu & $\begin{array}{c}\text { trifft völlig } \\
\text { zu }\end{array}$ \\
\hline Ich esse gerne Schokolade. & $\square$ & $\square$ & $\square$ & $\mathbf{X}$ \\
\hline
\end{tabular}

Hier müsstest Du „trifft völlig zu“ ankreuzen, wenn Du sehr gerne Schokolade isst. Dies ist im Beispiel der Fall. Wenn Du überhaupt keine Schokolade magst, müsstest Du „trifft gar nicht zu“ ankreuzen. Wenn Du Schokolade eher nicht magst, müsstest Du „trifft eher nicht zu“ ankreuzen; wenn Du Schokolade eher gerne magst, steht Dir die Antwort „trifft eher zu“ zur Verfügung.

\section{Beispiel 2:}

Spielst du gerne Tischtennis? Warum oder warum nicht?

ich spiele gerne Tischtennis, weil ich das ziemlich gut kann und weil man dieses Spiel

mit mehreren Freunden zusammen spielen kann. Auberdem haben wir in der schule eine

Tischtennisplatte und da haben wir oft die Gelegenheit zu üben.

Hier müsstest du selbst eine Antwort schreiben. 
Noch eine wichtige Sache:

Im ersten Teil des Fragebogens findest du auf jeder Seite einen Hinweis, ob du sofort auf die nächste Seite blättern sollst oder ob du noch warten sollst, bis du ein Zeichen zum Umblättern bekommst.

Das sieht dann so aus:

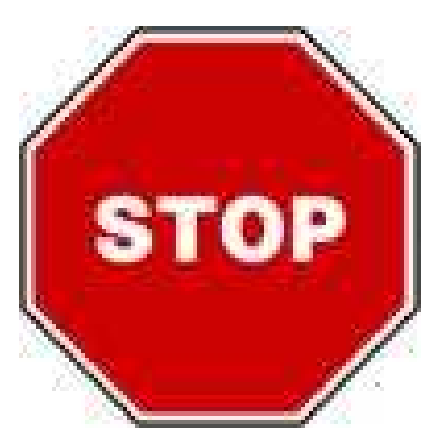

Warte, bis das Zeichen zum Umblättern kommt! 


\section{Die Freundschaft}

Marc und Jonas ${ }^{18}$ sind zwei gute Freunde in deinem Alter. Die beiden gehen in die gleiche Klasse. Sie sitzen in der Schule immer nebeneinander und verbringen oft ihre Freizeit gemeinsam. Entweder hören sie bei Marc Musik oder fahren auf ihren Inline-Skates durch ihr Viertel. Marc lacht gerne und hat viele Freunde. Bei ihm ist immer etwas los. Jonas ist sehr ruhig und sitzt gerne stundenlang alleine im Garten. Jonas' Eltern streiten sehr viel und haben kaum Zeit für ihn. Beide sind so sehr mit ihren Problemen beschäftigt, dass Jonas oft sich selbst überlassen wird. Nur Marc, der Jonas' einziger Freund ist, weiß davon, da Jonas seine Probleme für sich behalten will.

Eines Tages, als Jonas einkaufen geht und vor dem Supermarkt steht, trifft er Sven. Sven geht seit kurzem ebenfalls in ihre Klasse. Jonas hat bemerkt, dass Sven gerne mit Marc zusammen ist. Auf dem Heimweg schließt sich Sven immer den beiden an. Er spricht dann aber nur mit Marc, Jonas beachtet er nicht. Sven gesellt sich nun zu Jonas und erzählt ihm, dass Marc ihm alles über Jonas' Probleme erzählt habe. Jonas weiß zuerst nicht, was er sagen soll. Dann fragt er Sven, was Marc ihm denn genau erzählt habe. Sven meint darauf nur: „Ach, du weißt schon!“‘.

Jonas dreht sich schweigend um und geht direkt in den Supermarkt, ohne Sven weiter zu beachten. Er holt sich einen Einkaufswagen, packt die Einkaufsliste aus und fängt an, die Waren zusammenzusuchen. Er kann sich aber nicht recht konzentrieren und denkt immer wieder daran, was Sven gesagt hat. Plötzlich steht Marc mit einem Einkaufswagen vor ihm. Er lacht Jonas an und fragt, ob er nachher Lust habe, bei ihm zu Hause Musik zu hören. Jonas weiß nicht, was er tun soll und zögert mit einer Antwort. Marc merkt nun, dass mit Jonas etwas nicht stimmt und fragt, was los sei.

1. Frage: Was ist in dieser Geschichte das Problem? Warum ist das ein Problem?

2. Frage: Wie fühlt sich Jonas? Warum fühlt er sich so?

3. Frage: Wie fühlt sich Marc, als Jonas keine Antwort gibt? Warum fühlt er sich so?

${ }^{18}$ Den Mädchen wurde ein Szenario mit den Protagonistinnen Claudia, Sabine und Mirjam vorgelegt. 
4. Frage: Warum hat Sven Jonas erzählt, was Marc ihm gesagt haben soll?

5. Frage: Wie fühlt sich Sven? Warum fühlt er sich so?

6. Frage: Soll Jonas Marc darauf ansprechen, was Sven ihm erzählt hat? Warum (warum nicht)?

7. Frage: Was denkst Du, haben Jonas und Marc eine gute Freundschaft? Was ist eine gute Freundschaft?

8. Frage: Was für eine Person muss ein guter Freund sein? Warum muss er so sein?

9. Frage: Wie sollten gute Freunde eine Auseinandersetzung beenden? Warum sollten sie das tun? 
Beantworte bitte ein paar Fragen zu dir und deiner Familie. Die Antworten von dir werden von uns nicht an deinen Lehrer oder deine Lehrerin weitergegeben.

1. Bitte kreuze an, in welchem Land du geboren wurdest, in welchem Land deine Mutter und in welchem Land dein Vater geboren wurde!

\section{Du}

Deutschland

Türkei

Italien

Albanien

Bosnien

Kroatien

Serbien

Slowenien

Polen

Russland

Kasachstan

Frankreich

England

in einem anderen Land, und

zwar:

\section{Deine Mutter}

Deutschland

Türkei

Italien

Albanien

Bosnien

Kroatien

Serbien

Slowenien

Polen

Russland

Kasachstan

Frankreich

England

in einem anderen Land, und

zwar:

\section{Dein Vater}

Deutschland

Türkei

Italien

Albanien

Bosnien

Kroatien

Serbien

Slowenien

Polen

Russland

Kasachstan

Frankreich

England

in einem anderen Land, und

2. Falls du nicht in Deutschland geboren wurdest, wie alt warst du, als du nach Deutschland kamst? Jahre

\section{Welchen Schulabschluss haben deine Mutter und dein Vater?}

Bitte kreuze nur den böchsten Abschluss an!

\author{
Deine Mutter \\ keinen \\ Hauptschulabschluss \\ Realschulabschluss \\ Fachabitur \\ Abitur
}

\section{Dein Vater}

keinen

Hauptschulabschluss

Realschulabschluss

Fachabitur

Abitur

\section{Habt ihr regelmäßig eine Tageszeitung zu Hause? $\quad \square$ ja $\quad \square$ nein}
5. Wie oft kommt es vor, dass deine
nie oder
ein paar
Mal im
etwa
einmal im
mehrmals
im Monat
mehrmals
Jahr
Monat
in der
Bitte in jeder Zeile nur ein Kästchen ankreuzen. sehsendungen diskutieren?

b) mit dir klassische Musik hören?

c) mit dir über deine Schulleistungen sprechen?

d) gemeinsam mit dir am Tisch sitzen und zu Mittag oder Abend essen?

e) sich Zeit nehmen, um einfach nur mit dir zu reden?

\section{Wie viele Bücher gibt es ungefähr bei dir zu Hause?}



$\square$ keine
$\square \quad 1-10$
$\square \quad 11-50$
$51-100$
$101-250$
251-500
mehr als 500

Bei den folgenden Fragen geht es um deine Meinung über das Fach Geschichte

\begin{tabular}{|c|c|c|c|c|c|}
\hline & & $\begin{array}{l}\text { trifft gar } \\
\text { nicht zu }\end{array}$ & $\begin{array}{l}\text { trifft eher } \\
\text { nicht zu }\end{array}$ & $\begin{array}{c}\text { trifft } \\
\text { eher zu }\end{array}$ & $\begin{array}{c}\text { trifft völlig } \\
\mathrm{zu}\end{array}$ \\
\hline IG1 & Mir macht Geschichtsunterricht Spaß. & $\square$ & $\square$ & $\square$ & $\square$ \\
\hline SG4 & $\begin{array}{l}\text { Ich bin in Geschichte zufrieden mit meiner } \\
\text { Fähigkeit, vor der Klasse zu sprechen. }\end{array}$ & $\square$ & $\square$ & $\square$ & $\square$ \\
\hline$\overline{\mathrm{BG} 4}$ & $\begin{array}{l}\text { Meine Eltern interessieren sich für das, was ich } \\
\text { im Fach Geschichte lerne. }\end{array}$ & $\square$ & $\square$ & $\square$ & $\square$ \\
\hline$\overline{S G 8}$ & $\begin{array}{l}\text { Ich habe ein gutes Gefühl, was meine Arbeit in } \\
\text { Geschichte angeht. }\end{array}$ & $\square$ & $\square$ & $\square$ & $\square$ \\
\hline IG2 & $\begin{array}{l}\text { Ich lerne in Geschichte etwas, das für mich sehr } \\
\text { wichtig ist. }\end{array}$ & $\square$ & $\square$ & $\square$ & $\square$ \\
\hline$\overline{\text { SG1 }}$ & $\begin{array}{l}\text { Ich weiß in Geschichte die Antwort auf eine } \\
\text { Frage schneller als die anderen. }\end{array}$ & $\square$ & $\square$ & $\square$ & $\square$ \\
\hline$\overline{S G 6}$ & In Geschichte fallen mir gute Noten zu. & $\square$ & $\square$ & $\square$ & $\square$ \\
\hline$\overline{\mathrm{BG} 3}$ & $\begin{array}{l}\text { In unserer Wohnung gibt es Bücher oder Filme, } \\
\text { die etwas mit Geschichte zu tun haben. }\end{array}$ & $\square$ & $\square$ & $\square$ & $\square$ \\
\hline SG3 & $\begin{array}{l}\text { Ich kann in Geschichte Sachen selbst rauskrie- } \\
\text { gen. }\end{array}$ & $\square$ & $\square$ & $\square$ & $\square$ \\
\hline SG2 & $\begin{array}{l}\text { Manchmal fühle ich mich in Geschichte ande- } \\
\text { ren überlegen und glaube, dass sie noch man- } \\
\text { ches von mir lernen können. }\end{array}$ & $\square$ & $\square$ & $\square$ & $\square$ \\
\hline$\overline{B G 1}$ & $\begin{array}{l}\text { Manchmal sprechen wir in unserer Familie über } \\
\text { Ereignisse, die in der Geschichte passiert sind. }\end{array}$ & $\square$ & $\square$ & $\square$ & $\square$ \\
\hline$\overline{S G 5}$ & $\begin{array}{l}\text { Es fällt mir in Geschichte leicht, Probleme zu } \\
\text { lösen. }\end{array}$ & $\square$ & $\square$ & $\square$ & $\square$ \\
\hline $\mathrm{BG} 2$ & $\begin{array}{l}\text { Meine Eltern sagen mir, dass Geschichte ein } \\
\text { wichtiges Fach ist. }\end{array}$ & $\square$ & $\square$ & $\square$ & $\square$ \\
\hline SG7 & Ich gehöre in Geschichte zu den Guten. & $\square$ & $\square$ & $\square$ & $\square$ \\
\hline
\end{tabular}




\section{Was war deine letzte Zeugnisnote im Fach Geschichte? Kreuze an.}

$\begin{array}{ll}\square 1+ & \square 3- \\ \square 1 & \square 4+ \\ \square 1- & \square 4 \\ \square 2+ & \square 4- \\ \square 2 & \square 5+ \\ \square 2- & \square 5 \\ \square 3+ & \square 5- \\ \square 3 & \square 6\end{array}$

Bei den folgenden Fragen geht es darum, wie du dich im Alltag verhältst.

\begin{tabular}{|c|c|c|c|c|c|}
\hline & & $\begin{array}{l}\text { trifft gar } \\
\text { nicht zu }\end{array}$ & $\begin{array}{l}\text { trifft eher } \\
\text { nicht zu }\end{array}$ & $\begin{array}{c}\text { trifft } \\
\text { eher zu }\end{array}$ & $\begin{array}{c}\text { trifft völlig } \\
\mathrm{zu}\end{array}$ \\
\hline $\begin{array}{l}\text { PÜ1 } \\
\end{array}$ & $\begin{array}{l}\text { Bei Meinungsverschiedenheiten versuche ich, } \\
\text { die Sache aus Sicht aller Beteiligten zu betrach- } \\
\text { ten, bevor ich mich entscheide. }\end{array}$ & $\square$ & $\square$ & $\square$ & $\square$ \\
\hline SA6 & $\begin{array}{l}\text { Bevor ich aus dem Haus gehe, werfe ich einen } \\
\text { letzten Blick in den Spiegel. }\end{array}$ & $\square$ & $\square$ & $\square$ & $\square$ \\
\hline SA13 & $\begin{array}{l}\text { Es ist mir unangenehm, wenn andere mich } \\
\text { beobachten. }\end{array}$ & $\square$ & $\square$ & $\square$ & $\square$ \\
\hline PÜ4 & $\begin{array}{l}\text { Bevor ich Leute kritisiere, versuche ich mir } \\
\text { vorzustellen, wie es mir ginge, wenn ich an ihrer } \\
\text { Stelle wäre. }\end{array}$ & $\square$ & $\square$ & $\square$ & $\square$ \\
\hline$\overline{\text { SA12 }}$ & $\begin{array}{l}\text { Es ist mir wichtig, wie andere über mich den- } \\
\text { ken. }\end{array}$ & $\square$ & $\square$ & $\square$ & $\square$ \\
\hline$\overline{\text { SA11 }}$ & $\begin{array}{l}\text { Ich denke darüber nach, welchen Gesichtsaus- } \\
\text { druck ich gerade habe. }\end{array}$ & $\square$ & $\square$ & $\square$ & $\square$ \\
\hline SA7 & Ich achte auf mein Aussehen. & $\square$ & $\square$ & $\square$ & $\square$ \\
\hline SA9 & $\begin{array}{l}\text { Ich achte auf meine eigenen Bewegungen und } \\
\text { meine Körperhaltung. }\end{array}$ & $\square$ & $\square$ & $\square$ & $\square$ \\
\hline$\overline{\text { SA5 }}$ & $\begin{array}{l}\text { Ich achte darauf, dass ich in einem guten Licht } \\
\text { erscheine. }\end{array}$ & $\square$ & $\square$ & $\square$ & $\square$ \\
\hline SA1 & Ich achte darauf, wie ich aussehe. & $\square$ & $\square$ & $\square$ & $\square$ \\
\hline$\overline{\mathrm{SA} 2}$ & Ich betrachte mich gerne im Spiegel. & $\square$ & $\square$ & $\square$ & $\square$ \\
\hline SA4 & $\begin{array}{l}\text { Ich denke im Nachhinein darüber nach, wel- } \\
\text { chen Eindruck ich auf andere Menschen ge- } \\
\text { macht habe. }\end{array}$ & $\square$ & $\square$ & $\square$ & $\square$ \\
\hline PÜ2 & $\begin{array}{l}\text { Ich glaube, dass jedes Problem zwei Seiten hat, } \\
\text { und ich versuche, mir beide Seiten anzusehen. }\end{array}$ & $\square$ & $\square$ & $\square$ & $\square$ \\
\hline$\overline{\text { SA3 }}$ & $\begin{array}{l}\text { Ich mache mir Gedanken darüber, wie ich auf } \\
\text { andere Menschen wirke. }\end{array}$ & $\square$ & $\square$ & $\square$ & $\square$ \\
\hline SA8 & $\begin{array}{l}\text { Ich mache mir Gedanken darüber, wie ich mich } \\
\text { in Gegenwart anderer geben soll. }\end{array}$ & $\square$ & $\square$ & $\square$ & $\square$ \\
\hline SA10 & $\begin{array}{l}\text { Ich überlege, was meine Freunde und Bekannte } \\
\text { von mir denken. }\end{array}$ & $\square$ & $\square$ & $\square$ & $\square$ \\
\hline PÜ3 & $\begin{array}{l}\text { Ich versuche manchmal, meine Freunde besser } \\
\text { zu verstehen, indem ich mir vorstelle, wie die } \\
\text { Dinge aus ihrer Sicht aussehen. }\end{array}$ & $\square$ & $\square$ & $\square$ & $\square$ \\
\hline$\overline{\text { SA14 }}$ & Ich spüre es, wenn mich jemand beobachtet. & $\square$ & $\square$ & $\square$ & $\square$ \\
\hline PÜ5 & $\begin{array}{l}\text { Wenn ich mich über jemanden aufrege, versu- } \\
\text { che ich normalerweise erst einmal, mich in seine } \\
\text { Lage zu versetzen. }\end{array}$ & $\square$ & $\square$ & $\square$ & $\square$ \\
\hline
\end{tabular}




\section{Multiple Choice-Items und Lückentext für Klassenstufe 7}

Bitte beantworte nun die folgenden Fragen. Bei den meisten Fragen sollst du die richtige Antwort ankreuzen. Dort, wo du eine Linie siehst, sollst du selbst eine Antwort schreiben.

Frage 1: Welchen Zeitraum bezeichnet man als Mittelalter?
O ca. 1000 n. Chr. bis 1700 n. Chr.
O ca. 500 n. Chr. bis 1500 n. Chr.
O ca. 200 n. Chr. bis 900 n. Chr.
O ca. 800 n. Chr. bis 1200 n. Chr.

Frage 2: Mit welchem Ereignis fällt der Beginn des Mittelalters zusammen?
O mit dem Ende des weströmischen Reiches
O mit der Entdeckung Amerikas
O mit dem Ende des oströmischen Reiches
O mit der Ermordung Cäsars

Frage 3: Wer wurde im Mittelalter römischer Kaiser?
O der Papst in Rom
O der französische König
O der König von Rom
O der deutsche König

Frage 4: Welcher Kaiser wurde auch Barbarossa genannt?
O Friedrich I.
O Konrad II.
O Otto I.
O Heinrich II.

Frage 5: Die meisten Bauern im Mittelalter waren nicht frei, sondern gehörten einem Herrn. Wie nannte man diese Form der Unfreiheit?

Frage 6: Wie bezeichnet man die Dienste, die Bauern im Mittelalter für ihre Grundherren leisten mussten?

Frage 7: Wer rief zum ersten Kreuzzug auf? 

O der englische König Richard Löwenherz
O der deutsche Kaiser Friedrich Barbarossa
O Papst Urban
O Wilhelm der Eroberer

Frage 8: Wann begann der erste Kreuzzug?
O 1096
O 1247
O 988
O 1020

Frage 9: Wer unternahm 1077 den Gang nach Canossa?
O Otto I.
O Konrad II.
O Heinrich IV.
O Lothar III.

Frage 10: Welche drei Stände gab es im Mittelalter? Schreibe sie auf.

Frage 11: Wie nennt man das System, in dem im Mittelalter Herr und Vasall (Gefolgsmann) ein gegenseitiges Schutz- und Treueverhältnis eingingen?

Frage 12: Wie nennt man den Vorsteher eines Klosters im Mittelalter? 


\section{Bitte fülle nun noch den folgenden Lückentext aus. Dann hast du es geschafft!}

Das Mittelalter erstreckte sich über einen Zeitraum von ca. Jahren.

Einer der großen Herrscher des Mittelalters war Karl der Große. Am Weihnachtstag des Jahres wurde er in Rom zum Kaiser gekrönt.

Zu den mächtigen Männern im Mittelalter gehörten auch die geistlichen Würdenträger, nämlich die und die

Im Mittelalter lebten die meisten Menschen auf dem Land und waren Der Boden gehörte oft den Adligen und die Bauern mussten zahlen. Dafür standen sie unter dem des Grundherrn.

Viele Adlige des Mittelalters nahmen an den teil, zu denen der Papst aufrief. Die Pilger machten sich auf in die Stadt um sie von den Arabern zu befreien. 


\section{Multiple Choice-Items und Lückentext für Klassenstufe 10}

Bitte beantworte nun noch die folgenden Fragen. Bei den meisten Fragen sollst du die richtige Antwort ankreuzen. Dort, wo du eine Linie siehst, sollst du selbst eine Antwort schreiben.

Frage 1: Welcher Partei gehörte der erste Reichspräsident der Weimarer Republik Friedrich Ebert an?

Frage 2: Hindenburg versuchte nach dem Ende des 1. Weltkrieges, den linken Parteien die Schuld für die Niederlage zu geben. Mit welchem Begriff wird dieses Vorgehen bezeichnet?

Frage 3: Welches Ereignis geschah im Jahr 1923?

$\begin{array}{ll}\mathbf{O} & \text { Hitler-Putsch in München } \\ \mathbf{O} & \text { Börsencrash in New York } \\ \mathbf{O} & \text { Kapp-Putsch } \\ \mathbf{O} & \text { Unterzeichnung des Vertrags von Rapallo }\end{array}$

Frage 4: Wie nannte man die wirtschaftliche und kulturelle Blütezeit der Weimarer Republik von 1924 bis 1928 ?

Frage 5: In welchem Jahr übernahmen die Nationalsozialisten die Macht in Deutschland?

Frage 6: Wann ereignete sich der Börsencrash in New York, mit dem die Weltwirtschaftskrise begann?
O $\quad 1920$
O 1923
O 1929
O 1931

Frage 7: Wer ernannte Adolf Hitler zum Reichskanzler?

Frage 8: Was geschah in der Reichskristallnacht oder Reichspogromnacht?

O Machtergreifung durch Adolf Hitler 
O Zerstörung jüdischer Einrichtungen und Verhaftung von Juden

O Juden wurden gezwungen, den Judenstern zu tragen

O Bücherverbrennung durch die Nationalsozialisten

Frage 9: In welcher Reihenfolge wurden die folgenden Staaten im 2. Weltkrieg von Deutschland angegriffen?

$\begin{array}{ll}\text { O } & \text { Polen, Dänemark, Frankreich, Sowjetunion } \\ \text { O } & \text { Frankreich, Sowjetunion, Dänemark, Polen } \\ \text { O } & \text { Sowjetunion, Dänemark, Polen, Frankreich } \\ \text { O } & \text { Dänemark, Sowjetunion, Frankreich, Polen }\end{array}$

Frage 10: Welche der folgenden Staaten gehörten zu den Alliierten im 2. Weltkrieg?

$\begin{array}{ll}\text { O } & \text { Großbritannien, USA, Jugoslawien } \\ \text { O } & \text { Großbritannien, USA, Deutschland } \\ \text { O } & \text { Großbritannien, USA, Niederlande } \\ \text { O } & \text { Großbritannien, USA, Sowjetunion }\end{array}$

Frage 11: In wie viele Besatzungszonen wurde Deutschland nach dem 2. Weltkrieg aufgeteilt?

In __ Besatzungszonen

Frage 12: In welchem Jahr wurden die Bundesrepublik Deutschland und die Deutsche Demokratische Republik gegründet?

$\begin{array}{ll}\mathrm{O} & 1946 \\ \mathrm{O} & 1949 \\ \mathrm{O} & 1952 \\ \mathrm{O} & 1955\end{array}$




\section{Bitte fülle nun noch den folgenden Lückentext aus. Dann hast du es geschafft!}

Nach dem ersten Weltkrieg wurde im Jahr die Weimarer Republik gegründet.

Deutschland war nun keine Monarchie mehr, sondern eine In der Folgezeit gab es viele Schwierigkeiten. Im Krisenjahr spitzten sich die Probleme zu. Dazu gehörte der Ruhrkampf, in dem es um die Auseinandersetzung mit den Franzosen um die ging. Nach einigen wirtschaftlich und kulturell positiven Jahren erschütterte der Börsencrash mit seinen schweren wirtschaftlichen Folgen die Welt. Dies waren aber nur zwei von vielen wirtschaftlichen und politischen Ereignissen, die die Weimarer Republik zum Scheitern führten.

Am 30. Januar ernannte Reichspräsident

zum Reichskanzler. Schon nach kurzer Zeit gelang Hitler die Adolf Hitler Begriff der Nationalsozialisten - der Parteien und Verbände und er errichtete eine totalitäre

Durch den Überfall auf im Jahre 1939 entfesselte er den 2. Weltkrieg. Nun steigerten sich die bereits vor dem Krieg begonnenen Judenverfolgungen zum Massenmord an ca. 6 Millionen Menschen. Die Übermacht der Alliierten erzwang schließlich die bedingungslose Kapitulation Deutschlands.

Nach der Kapitulation des Reiches ging die Regierungsgewalt Deutschlands vollständig an die Alliierten über. Deutschland wurde unter den Siegermächten aufgeteilt. Während in der Zone das sozialistische Gesellschaftsmodell durchgesetzt wurde und schließlich die entstand, wurden die übrigen Zonen zum Staatsgebiet der Deutschland vereinigt. 